\title{
Freshwater fish distribution in the Maghreb: a call to contribute
}

Matthew Ford ${ }^{1 *}$, Amina Brahimi², Lyas Baikeche ${ }^{3}$, Laura Bergner ${ }^{4}$, Miguel Clavero ${ }^{5}$, Ignacio Doadrio6, Manuel Lopes-Lima ${ }^{7}$, Silvia Perea ${ }^{6}$, Ahmed Yahyaoui ${ }^{8}$ and Jörg Freyhof ${ }^{1}$

${ }^{1}$ Centre for Integrative Biodiversity Discovery, Museum für Naturkunde, Invalidenstrasse 43, 10115 Berlin, Germany.

${ }^{2}$ Zoogeography Research Unit, Department of Biology, Ecology and Evolution, University of Liège, 4000 Liège, Belgium.

${ }^{3}$ Faculty of Biological Sciences and Agricultural Sciences, Mouloud Mammeri University, BP 15000 Tizou Ouzou, Algeria.

${ }^{4}$ Leibniz-Institute of Freshwater Ecology and Inland Fisheries (IGB), Müggelseedamm 310, 12587 Berlin, Germany.

${ }^{5}$ Estación Biológica de Doñana, Cartuja TA-10, Edificio I, Calle Américo Vespucio, s/n, 41092 Sevilla, Spain.

${ }^{6}$ Biodiversity and Evolutionary Group, Museo Nacional de Ciencias Naturales, CSIC. C/José Gutiérrez Abascal, 2, 28006 Madrid, Spain.

${ }^{7}$ CIBIO-InBIO Research Centre in Biodiversity and Genetic Resources, University of Porto, 4485-661 Vairão, Portugal.

${ }^{8}$ Laboratory of Biodiversity, Ecology and Genome, Faculty of Sciences, Mohammed V University in Rabat. B.P. 1014 Rabat, Morocco.

${ }^{*}$ Corresponding author: Matthew Ford. E-mail: matthew.ford@mfn.berlin.

\section{Acknowledgements}

This project has received funding from the European Union's Horizon 2020 research and innovation programme under the Marie Skłodowska-Curie grant agreement No 766417.

\section{Abstract}

In order to update the IUCN Red List of all freshwater fish species inhabiting the Maghreb region, comprehensive knowledge of their respective geographic ranges is essential. Here we present site-scale distribution records derived from all known sources for native and alien freshwater fish species distributed in Morocco, Algeria and Tunisia. Corrected data compiled from the Global Biodiversity Information Facility plus author contributions and digitised literature data are presented as GIS maps, and the wider scientific and conservation communities are called upon to help fill knowledge gaps and maximise the value of this new database for a region in which freshwater biodiversity is undergoing a serious decline. 
Keywords: freshwater fish, IUCN Red List, biodiversity, conservation, Maghreb, North Africa

\section{Introduction}

Freshwater ecosystems in the Maghreb, defined here as the region delimited by the North African countries Morocco (excluding the territories of Ceuta, Melilla and Western Sahara), Algeria and Tunisia, face severe anthropogenic and environmental pressures (Fouchy et al., 2019). These stresses are driven primarily by the water demands of a rapidly expanding human population in one of the most arid regions of the world, where water reserves are consistently lower than the world average of renewable water per inhabitant (Oualkacha et al., 2017; FAOSTAT, 2019). Despite these challenging conditions the Maghreb harbours a rich diversity of freshwater fish species with largely Palearctic relationships (Doadrio, 1994; García et al., 2010).

The last comprehensive IUCN Red List of Threatened Species (hereafter Red List) assessment of extinction risk for freshwater ichthyofauna of the Maghreb was published in 2006. However, such assessments must be updated at least once every ten years to ensure their accuracy and prevent them being declared as outdated (Rodrigues et al., 2006; Rondinini et al., 2014). The IUCN has devised a series of formal criteria for Red List assessments among which the delineation of a species' geographic extent is a central component (IUCN, 2012). Uncertainties about precisely where species are distributed can thus constitute a major limiting factor in the evaluation process (Pimm et al., 2014). In addition, knowledge of species ranges is critical for informing progress towards several of the Convention on Biological Diversity's Aichi biodiversity targets and related environmental indicators, as well as assessment efforts undertaken by the Intergovernmental Science-Policy Platform on Biodiversity and Ecosystem Services (Pereira et al., 2013; Brooks et al., 2015; Meyer et al., 2015).

The distribution of freshwater fishes in the Maghreb was first studied in detail during the early 1900s (Boulenger, 1911; Pellegrin, 1921). Almost a century later an IUCN-led project produced as an output coarse species-range polygons based partly on maps which first appeared in studies focused on the freshwater fishes of Morocco (Azeroual et al., 2000; Azeroual, 2003; García et al., 2010). The distribution of threatened species was subsequently reviewed in order to evaluate proposed Key Biodiversity Areas, i.e., areas contributing significantly to the global persistence of biodiversity (Darwall et al., 2014).

Recent molecular and taxonomic studies have further highlighted the richness of freshwater fish species diversity in the region leading to an improved understanding of their occurrence and distribution, albeit significant knowledge gaps remain (Geiger et al., 2014; Casal-Lopez et al., 2015; Doadrio et al., 2015, 2016a,b; Brahimi et al. 2016, 2017, 2018; Touil et al., 2019).

The Global Biodiversity Information Facility (henceforth GBIF) is the primary international resource offering access to species occurrence records derived from a variety of sources, particularly museum collections and field inventories (Beck et al., 2013). However, socio-economic and geographic disparities in funding and data sharing conventions have led to taxonomic, thematic, geospatial and temporal biases, gaps and errors in the available data (Yesson et al., 2007; Gaiji et al., 2013). Citizen science is a potentially effective approach to amassing data over different geographic 
and temporal scales (Dickinson et al., 2012; Amano et al., 2016). Many ground-level researchers, conservationists, agencies and groups based in the Maghreb remain unaware of the current Red List reassessment project. We therefore call upon this wider community to contribute by exchanging knowledge in order to develop a more authoritative Red List for freshwater fishes in the region and aid well-informed planning of future conservation agendas and priorities at both local and national scales.

This study is neither complete nor definitive so we also invite comments highlighting inaccuracies or offering constructive feedback.

\section{Methods}

We conducted an extensive literature review and consulted with local and international experts before compiling an inventory of all native and alien fish species known to occur in freshwaters of the Maghreb region. All available GBIF point occurrence records for each of these species were then downloaded as individual shapefiles. These spatial data were combined with our own field sampling records plus georeferenced records from existing published literature (Boutellier, 1918; Dollfus, 1955; Furnestin et al., 1958; Libois et al., 1997, 2015; Azeroual, 2003; Bacha \& Amar, 2007; Nait-Larbi, 2011; Qninba et al., 2011, 2012; Renaud, 2011; Clavero et al., 2014; Mimeche \& Oliva-Paterna, 2018). The territories of Ceuta, Melilla and Western Sahara were subsequently omitted from the study due to a lack of data.

All data were carefully checked for credibility, nomenclature and taxonomy. Records with questionable dates, taxonomy, mismatches between coordinates and country of origin, and those located outside the study region were removed. The final data set was visualised on individual regional-scale maps for each species using the open source Geographic Information System QGIS version 3.4 (QGIS, 2019), with each site-scale record displayed as a point overlaying world imagery (Esri, 2019) and inland water (Lehner et al., 2008) base layers.

A second series of stringent quality control checks were carried out during May 2019 at an IUCN Red List workshop in Rabat, Morocco, at which time we reviewed all the collated data.

Nomenclature and taxonomy mostly follows Eschmeyer's Catalog of Fishes (Fricke et al., 2019). However, according to recent molecular studies Anaecypris punica does not belong to the genus Phoxinellus and is most closely related to the Iberian endemic Anaecypris hispanica (Perea et al., 2010; Geiger et al., 2014). In addition, Salmo macrostigma is a junior synonym of Salmo cettii (Splendiani et al., 2019).

\section{Results and discussion}

Including diadromous and euryhaline taxa, 62 species were recognised as native and 24 species as non-native to the region (Table 1). Among the native species, 41 were considered endemic.

A total of 2050 site scale records were identified from the aforementioned sources (Fig. 1). In some cases the data accurately reflected species' known geographic ranges, but in the majority there were significant gaps in the distribution records (Figs. 2-87). 
This was especially true for native freshwater fishes in Tunisia, where very few records exist even for widespread taxa such as Luciobarbus callensis but especially range-restricted species like Anaecypris punica, Tropidophoxinellus callensis or T. chaignoni. Moreover, Anaecypris punica is also expected to occur in Algeria but confirmatory records have yet to be obtained. Vast expanses of Algeria and Morocco remain poorly sampled which can at least in part be explained by the paucity of local contributions to our data set.

We did not at this stage take into consideration that some GBIF data is quite old and may not reflect current distributions, since we later intend to generate polygons depicting the extant, extirpated, extinct and alien ranges (as applicable) for each taxon. Wide-ranging species such as Acipenser sturio and Alosa alosa have been extirpated from the region, while endemics including Aphanius apodus, $A$. saourensis, Luciobarbus antinorii, L. nasus, and Salmo pallaryi are extinct or extinct in the wild. Accurate time series' or other forms of population trend data for freshwater fishes of the Maghreb were lacking and could not be taken into account. Contributions from the wider community to further knowledge in these respects would therefore be most welcome.

How can I contribute? There are two major pathways to support this project and help improve the Red List for freshwater fishes of the Maghreb:

1. Publish your data. Interested contributors could publish their species distribution data in a peer-reviewed journal and make the data available through an online platform such as ResearchGate (www.researchgate.net). There are journals specialising in publication of biodiversity data, including the Biodiversity Data Journal (https://bdj.pensoft.net), and some dedicated ichthyological journals accept manuscripts describing the distribution of freshwater fishes. Some of these include:

- Ichthyological Exploration of Freshwaters (https://pfeil-verlag.de),

- Journal of Applied Ichthyology (https://onlinelibrary.wiley.com/journal/14390426)

- Croatian Journal of Fisheries (https://ribarstvo.agr.hr)

2. Submit your data to us. You can contact the lead author of this study (MF) to contribute your data directly, with even limited data sets considered valuable. Data should be submitted in Excel format, for which a template will be made available to interested parties.

In all cases, it is essential that individual species records are submitted alongside GPS coordinates, ideally in decimal format, so that they can be added to the existing maps. Colour photographs taken in lateral view should accompany data for less easily-identified species in order that they can be reviewed.

\section{Literature cited}

Amano, T., Lamming, J.D. and Sutherland, W.J. (2016). Spatial gaps in global biodiversity information and the role of citizen science. Bioscience 66(5): 393-400.

Azeroual, A., Crivelli, A.J., Yahyaoui, A. and Dakki, M. (2000). L'ichtyofaune Des Eaux Continentales

Du Maroc. Cybium 24(3) suppl.: 17-22. 
Azeroual, A. (2003). Monographie des poissons des eaux continentales du Maroc: systématique, distribution et écologie. Thès de Doctorat, Université Mohammed V-Agdal, Rabat: 1-193.

Bacha, M. and Amara, R. (2007). Les poissons des eaux continentales d'Algérie. Étude de l'ichtyofaune de la Soummam. Cybium 31(3): 351-358.

Beck, J., Ballesteros-Mejia, L., Nagel, P. and Kitching, I.J. (2013). Online solutions and the 'Wallacean shortfall': what does GBIF contribute to our knowledge of species' ranges? Diversity and Distributions 19(8): 1043-1050.

Boulenger, G.A. (1911). Catalogue of the fresh-water fishes of Africa in the British Museum (Natural History). London. v. 2: i-xii + 1-529.

Boutellier P. (1918). La Pêche maritime au Maroc (Casablanca, Fédala, Rabat). Casablanca: G. Mercié \& Co.: 1-94.

Brahimi, A., Tarai, N., Benhassane, A., Henrard, A. and Libois, R. (2016). Genetic and morphological consequences of Quaternary glaciations: A relic barbel lineage (Luciobarbus pallaryi, Cyprinidae) of Guir Basin (Algeria). Comptes Rendus Biologies 339(2): 83-98.

Brahimi, A., Freyhof, J., Henrard, A., and Libois, R. (2017). Luciobarbus chelifensis and L. mascarensis, two new species from Algeria (Teleostei: Cyprinidae). Zootaxa 4277(1): 32-50.

Brahimi, A., Libois, R., Henrard, A. and Freyhof, J. (2018). Luciobarbus lanigarensis and L. numidiensis, two new species of barbels from the Mediterranean Sea basin in North Africa (Teostei [sic Teleostei]: Cyprinidae). Zootaxa 4433(3): 542-560.

Brooks, T.M., Butchart, S.H., Cox, N.A., Heath, M., Hilton-Taylor, C., Hoffmann, M., Kingston, N., Rodríguez, J.P., Stuart, S.N. and Smart, J. (2015). Harnessing biodiversity and conservation knowledge products to track the Aichi Targets and Sustainable Development Goals. Biodiversity 16(2-3): 157-174.

Casal-Lopez, M., Perea, S., Yahyaoui, A. and Doadrio, I. (2015). Taxonomic review of the genus Luciobarbus Haeckel, 1843 (Actinopterygii, Cyprinidae) from Northwestern Morocco with the description of three new species. Graellsia 71(2): e027.

Clavero, M., Adrados, B., Calzada, J. and Jacome-Flores, M. (2014). On the presence of Petromyzon marinus in Oued Moulouya (Morocco). Cybium 38(4): 307-308.

Darwall, W., Carrizo, S., Numa, C., Barrios, V., Freyhof, J. and Smith, K. (2014). Freshwater Key Biodiversity Areas in the Mediterranean Basin Hotspot: Informing species conservation and development planning in freshwater ecosystems. Cambridge, UK and Malaga, Spain: IUCN. i-X + $1-86$.

Dickinson, J.L., Shirk, J., Bonter, D., Bonney, R., Crain, R.L., Martin, J., Phillips, T. and Purcell, K. (2012). The current state of citizen science as a tool for ecological research and public engagement. Frontiers in Ecology and the Environment 10(6): 291-297.

Doadrio, I. (1994). Freshwater fish fauna of North Africa and its biogeography. Annales-Musee Royal de l'Afrique Centrale 275: 21-34. 
Doadrio, I., Perea, S. and Yahyaoui, A. (2015). Two new species of Atlantic trout (Actinopterygii, Salmonidae) from Morocco. Graellsia 71(2): e031.

Doadrio, I., Casal-Lopez, M., Perea, S. and Yahyaoui, A. (2016a). Taxonomy of rheophilic Luciobarbus Haeckel, 1842 (Actinopterygii, Cyprinidae) from Morocco with the description of two new species. Graellsia 72(1): e039.

Doadrio, I., Casal-Lopez, M. and Perea, S. (2016b). Taxonomic remarks on Barbus moulouyensis Pellegrin, 1924 (Actinopterygii, Cyprinidae) with the description of a new species of Luciobarbus Haeckel, 1843 from Morocco. Graellsia 72(2): e054.

Dollfus, R.P. (1955). Première contribution a l'éstablissement d'un fichier ichthyologique du Maroc atlantique de Tanger à l'embouchure de l'Oued Dra. Travaux de l'Institut Scientifique Chérifien, Série Zoologie No. 6: 1-227.

Esri. (2019). World Imagery. December 15, 2019. Available online at: http://services.arcgisonline.com/ArcGIS/rest/services/World_Imagery/MapServer.

FAOSTAT 2019. Available online at: http://www.fao.org/faostat. Accessed 30/11/2019.

Fouchy, K., McClain, M.E., Conallin, J. and O'Brien, G. (2019). Chapter 10. Multiple Stressors in African Freshwater Systems. Pp. 179-191. In: Multiple Stressors in River Ecosystems: Status, Impacts and Prospects for the Future. Elsevier. 1-404.

Fricke, R., Eschmeyer, W.N. and Van der Laan, R. (eds). (2019). Eschmeyer's Catalog of Fishes: genera, species, references. Available online at: http://researcharchive.calacademy.org/research/ichthyology/catalog/fishcatmain.asp. Accessed 05/11/2019.

Furnestin, J., Dardignac, J., Maurin, C., Vincent, A., Coupe, R. and Boutiere, H. (1958). Données nouvelles sur les poissons du Maroc atlantique. Revue des travaux de l'Institut des pêches maritimes 22: 379-493.

Gaiji, S., Chavan, V., Ariño, A.H., Otegui, J., Hobern, D., Sood, R. and Robles, E. (2013). Content assessment of the primary biodiversity data published through GBIF network: status, challenges and potentials. Biodiversity Informatics 8(2): 94-172.

García, N., Abdul Malak, D., Kraïem, M., Samraoui, B., Azeroual, A., Cuttelod, A., Reda Fishar M., Yahyaoui, A. and Melhaoui, M. (2010). Chapter 3. The status and distribution of freshwater fish. Pp. 14-28. In: García, N., Cuttelod, A. and Abdul Malak, D. (eds.) (2010). The Status and Distribution of Freshwater Biodiversity in Northern Africa. Gland, Switzerland, Cambridge, UK, and Malaga, Spain: IUCN, 2010. i-xiii + 1-141.

GBIF.org. (2019). Available online at: http://www.gbif.org

Geiger, M.F., Herder, F., Monaghan, M.T., Almada, V., Barbieri, R., Bariche, M., Berrebi, P., Bohlen, J., Casal-Lopez, M., Delmastro, G.B., Denys, G.P. J., Dettai, A., Doadrio, I., Kalogianni, E., Kärst, H., Kottelat, M., Kovačić, M., Laporte, M., Lorenzoni, M., Marčić, Z., Özuluğ, M., Perdices, A., Perea, S., Persat, H., Porcelotti, S., Puzzi, C., Robalo, J., Šanda, R., Schneider, M., Šlechtová, V., Stoumboudi, M., Walter, S. and Freyhof, J. (2014). Spatial heterogeneity in the Mediterranean 
Biodiversity Hotspot affects barcoding accuracy of its freshwater fishes. Molecular Ecology Resources 14(6): 1210-1221.

Gozlan, R.E., Andreou, D., Asaeda, T., Beyer, K., Bouhadad, R., Burnard, D., Caiola, N., Cakic, P., Djikanovic, V., Esmaeili, H.R., Falka, I., Golicher, D., Harka, A., Jeney, G., Kovác, V., Musil, J., Nocita, A., Povz, M., Poulet, N., Virbickas, T., Wolter, C., Serhan Tarkan, A., Tricarico, E., Trichkova, T., Verreycken, H., Witkowski, A., Zhang, C-G., Zweimueller, I. and Britton, R.J. (2010). Pan-continental invasion of Pseudorasbora parva: towards a better understanding of freshwater fish invasions. Fish and Fisheries 11(4): 315-340.

IUCN. (2012). IUCN Red List Categories and Criteria: Version 3.1. Second edition. Gland, Switzerland and Cambridge, UK: IUCN. i-iv + 1-32.

Lehner, B., Verdin, K. and Jarvis, A. (2008): New global hydrography derived from spaceborne elevation data. Eos, Transactions, AGU 89(10): 93-94.

Libois, R., Ghalmi, R. and Brahimi, A. (2015). Insight into the Dietary Habits of the Eurasian Otter, Lutra lutra, in the East of Algeria (El-Kala National Park). Ecologia Mediterranea 41(2): 85-91.

Libois, R., Fareh, M., Brahimi, A. and Rosoux, R. (2015). Régime alimentaire et stratégie trophique saisonnière de la Loutre d'Europe, Lutra lutra, dans le Moyen Atlas (Maroc). Revue d'Écologie (Terre et Vie) 70(4): 314-327.

Meyer, C., Kreft, H., Guralnick, R. and Jetz, W. (2015). Global priorities for an effective information basis of biodiversity distributions. Nature Communications 6: 8221.

Mimeche, F. and Oliva-Paterna, F.J. (2018). Temporal variations in abundance and biomass of fish species inhabiting the K'sob reservoir (Eastern of Algeria). Studia Universitatis Babeş-Bolyai Biologia LXIII(2): 131-138.

Nait-Larbi, H. (2011). Utilisation des ressources alimentaires par la loutre d'Europe, Lutra lutra (Linné, 1758) durant deux saisons (été - automne) dans le barrage de Djorf - Torba (Kenadsa - BECHAR). Département Zoologie Agricole, École Nationale Supérieure Agronomique, Algiers. Unpublished magister thesis. 1-55.

Oualkacha, L., Stour, L., Agoumi, A. and Kettab, A. (2017). Climate change impacts in the Maghreb region: status and prospects of the water resources. Pp. 17-25. In: Ouessar M., Gabriels D., Tsunekawa A. and Evett S. (eds.). Water and Land Security in Drylands. Springer, Cham, Switzerland. i-xvii + 1-348.

Pellegrin, J. (1921). Les poissons des eaux douces de l'Afrique du Nord Francaise, Maroc, Algerie, Tunisie, Sahara. Mémoires de la Société des Sciences Naturelles du Maroc 1: 1-216.

Perea, S., Böhme, M., Zupančič, P., Freyhof, J., Šanda, R., Özuluğ, M., Abdoli, A. and Doadrio, I. (2010). Phylogenetic relationships and biogeographical patterns in Circum-Mediterranean subfamily Leuciscinae (Teleostei, Cyprinidae) inferred from both mitochondrial and nuclear data. BMC Evolutionary Biology 10(265): 1-27.

Pereira, H.M., Ferrier, S., Walters, M., Geller, G.N., Jongman, R.H.G., Scholes, R.J., Bruford, M.W., Brummitt, N., Butchart, S.H.M., Cardoso, A.C., Coops, N.C., Dullo, E., Faith, D.P., Freyhof, J., Gregory, R.D., Heip, C., Höft, R., Hurtt, G., Jetz, W., Karp, D.S., McGeoch, M.A., Obura, D., 
Onoda, Y., Pettorelli, N., Reyers, B., Sayre, R., Scharlemann, J.P.W., Stuart, S.N., Turak, E., Walpole, M. and Wegmann, M. (2013). Essential Biodiversity Variables. Science 339(6117): 277278.

Pimm, S.L., Jenkins, C.N., Abell, R., Brooks, T.M., Gittleman, J.L., Joppa, L.N., Raven, P.H., Roberts, C.M. and Sexton, J.O. (2014). The biodiversity of species and their rates of extinction, distribution, and protection. Science 344(6187): 1246752.

QGIS Development Team (2019). QGIS Geographic Information System. Open Source Geospatial Foundation Project. Available online at: http://qgis.osgeo.org.

Qninba, A., Lieron, V., Dieuleveut, T., Amairat, M. and Yahyaoui, A. (2011). Sur la présence de l'Anguille Anguilla anguilla (Linnaeus, 1758) dans l'Oued Tissint, un affluent de l'Oued Dr'a (Maroc). Bulletin de l'Institut Scientifique, Rabat, section Sciences de la Vie 33(2): 65-66.

Qninba, A., El Agbani, M.A., Radi, M. and Pariselle, A. (2012). Sur la présence de Tilapia guineensis (Teleostei, Cichlidae) dans les gueltas d'un affluent de l'Oued Chbeyka, l'Oued Aabar (Province de Tan Tan, Sud-ouest du Maroc). Bulletin Institut Scientifique, Rabat, section Sciences de la Vie 34(2): 125-126.

Renaud, C.B. (2011). Lampreys of the world. An annotated and illustrated catalogue of lamprey species known to date. FAO Species Catalogue for Fishery Purposes. No. 5. Rome, FAO. 1-109.

Rodrigues, A.S., Pilgrim, J.D., Lamoreux, J.F., Hoffmann, M. and Brooks, T.M. (2006). The value of the IUCN Red List for conservation. Trends in Ecology and Evolution 21(2): 71-76.

Rondinini, C., Di Marco, M., Visconti, P., Butchart, S.H. and Boitani, L. (2014). Update or outdate: Long-term viability of the IUCN Red List. Conservation Letters 7(2): 126-130.

Splendiani, A., Palmas, F., Sabatini, A. and Caputo Barucchi, V. (2019). The name of the trout: considerations on the taxonomic status of the Salmo trutta L., 1758 complex (Osteichthyes: Salmonidae) in Italy. The European Zoological Journal 86(1): 432-442.

Touil, A., Casal-Lopez, M., Bouhadad, R. and Doadrio, I. (2019). Phylogeny and phylogeography of the genus Luciobarbus (Haeckel, 1843) in Algeria inferred from mitochondrial DNA sequence variation. Mitochondrial DNA Part A 30(2): 332-344.

Yesson, C., Brewer, P.W., Sutton, T., Caithness, N., Pahwa, J.S., Burgess, M., Gray, W.A., White, R.J., Jones, A.C., Bisby, F.A. and Culham, A. (2007). How global is the global biodiversity information facility? PloS One 2(11): e1124. 
Table 1: Freshwater fish fauna of the Maghreb, including diadromous, euryhaline and alien species.

\begin{tabular}{|c|c|c|c|}
\hline Family & Species & Native & Alien \\
\hline Petromyzontidae & Petromyzon marinus Linnaeus 1758 & $x$ & \\
\hline Acipenseridae & Acipenser sturio Linnaeus, 1758 & $x$ & \\
\hline Anguillidae & Anguilla anguilla Linnaeus, 1758 & $x$ & \\
\hline \multirow[t]{3}{*}{ Clupeidae } & Alosa algeriensis Regan, 1916 & $x$ & \\
\hline & Alosa alosa (Linnaeus, 1758) & $x$ & \\
\hline & Alosa fallax (Lacepède, 1803) & $x$ & \\
\hline \multirow[t]{29}{*}{ Cyprinidae } & Carassius auratus (Linnaeus, 1758) & & $x$ \\
\hline & Carassius langsdorfii Temminck \& Schlegel, 1846 & & $x$ \\
\hline & Carasobarbus fritschii (Günther, 1874) & $x$ & \\
\hline & Carasobarbus harterti (Günther, 1901) & $x$ & \\
\hline & Cyprinus carpio Linnaeus, 1758 & & $x$ \\
\hline & Labeobarbus reinii (Günther, 1874) & $x$ & \\
\hline & Luciobarbus antinorii (Boulenger, 1911) & $x$ & \\
\hline & Luciobarbus biscarensis (Boulenger, 1911) & $x$ & \\
\hline & Luciobarbus callensis (Valenciennes, 1842) & $x$ & \\
\hline & Luciobarbus chelifensis Brahimi, Freyhof, Henrard \& Libois, 2017 & $x$ & \\
\hline & Luciobarbus figuiguensis (Pellegrin, 1913) & $x$ & \\
\hline & Luciobarbus guercifensis Doadrio, Perea \& Yahyaoui, 2016 & $x$ & \\
\hline & Luciobarbus ksibi Boulenger, 1905 & $x$ & \\
\hline & Luciobarbus labiosa (Pellegrin, 1920) & $x$ & \\
\hline & Luciobarbus lanigarensis Brahimi, Libois, Henrard \& Freyhof, 2018 & $x$ & \\
\hline & Luciobarbus lepineyi (Pellegrin, 1939) & $x$ & \\
\hline & Luciobarbus leptopogon (Schimper, 1834) & $x$ & \\
\hline & Luciobarbus magniatlantis (Pellegrin, 1919) & $x$ & \\
\hline & Luciobarbus mascarensis Brahimi, Freyhof, Henrard \& Libois, 2017 & $x$ & \\
\hline & Luciobarbus massaensis (Pellegrin, 1922) & $x$ & \\
\hline & Luciobarbus nasus (Günther, 1874) & $x$ & \\
\hline & Luciobarbus numidiensis Brahimi, Libois, Henrard \& Freyhof, 2018 & $x$ & \\
\hline & Luciobarbus pallaryi (Pellegrin, 1919) & $x$ & \\
\hline & Luciobarbus rabatensis Doadrio, Perea \& Yahyaoui, 2015 & $x$ & \\
\hline & Luciobarbus rifensis Doadrio, Casal-López \& Yahyaoui, 2015 & $x$ & \\
\hline & Luciobarbus setivimensis (Valenciennes, 1842) & $x$ & \\
\hline & Luciobarbus yahyaouii Doadrio, Casal-López \& Perea, 2016 & $x$ & \\
\hline & Luciobarbus zayanensis Doadrio, Casal-López \& Yahyaoui, 2016 & $x$ & \\
\hline & Pterocapoeta maroccana Günther, 1902 & $x$ & \\
\hline \multirow[t]{3}{*}{ Xenocyprididae } & Ctenopharyngodon idella (Valenciennes, 1844) & & $x$ \\
\hline & Hypophthalmichthys molitrix (Valenciennes, 1844) & & $x$ \\
\hline & Hypophthalmichthys nobilis (Richardson, 1845) & & $x$ \\
\hline \multirow[t]{5}{*}{ Leuciscidae } & Anaecypris punica (Pellegrin, 1920) & $x$ & \\
\hline & Rutilus rubilio (Bonaparte, 1837) & & $x$ \\
\hline & Rutilus rutilus (Linnaeus, 1758) & & $x$ \\
\hline & Tropidophoxinellus callensis (Guichenot, 1850) & $\mathrm{x}$ & \\
\hline & Tropidophoxinellus chaignoni (Vaillant, 1904) & $x$ & \\
\hline
\end{tabular}


Table 1: continued.

\begin{tabular}{|c|c|c|c|}
\hline Family & Species & Native & Alien \\
\hline & Scardinius erythrophthalmus (Linnaeus, 1758) & & $x$ \\
\hline & Squalius cephalus (Linnaeus, 1758) & & $\mathrm{x}$ \\
\hline Tincidae & Tinca tinca (Linnaeus, 1758) & & $x$ \\
\hline Cobitidae & Cobitis maroccana Pellegrin, 1929 & $x$ & \\
\hline \multirow[t]{2}{*}{ Gobionidae } & Gobio gobio (Linnaeus, 1758) & & $x$ \\
\hline & Pseudorasbora parva (Temminck \& Schlegel, 1846) & & $x$ \\
\hline \multirow[t]{2}{*}{ Clariidae } & Clarias anguillaris (Linnaeus, 1758) & $x$ & \\
\hline & Clarias gariepinus (Burchell, 1822) & $x$ & \\
\hline Esocidae & Esox lucius Linnaeus, 1758 & & $\mathrm{x}$ \\
\hline \multirow[t]{9}{*}{ Salmonidae } & Oncorhynchus mykiss (Walbaum, 1792) & & $x$ \\
\hline & Salmo akairos Delling \& Doadrio, 2005 & $x$ & \\
\hline & Salmo cettii (Rafinesque, 1810) & $x$ & \\
\hline & Salmo multipunctata Doadrio, Perea \& Yahyaoui, 2015 & $x$ & \\
\hline & Salmo pallaryi Pellegrin, 1924 & $x$ & \\
\hline & Salmo pellegrini Werner, 1931 & $x$ & \\
\hline & Salmo cf. trutta Linnaeus, 1758 & & $\mathrm{x}$ \\
\hline & Salmo viridis Doadrio, Perea \& Yahyaoui, 2015 & $x$ & \\
\hline & Salvelinus fontinalis (Mitchill, 1814) & & $x$ \\
\hline \multirow[t]{5}{*}{ Mugilidae } & Chelon auratus (Risso, 1810) & $x$ & \\
\hline & Chelon labrosus (Risso, 1827) & $x$ & \\
\hline & Chelon ramada (Risso, 1827) & $x$ & \\
\hline & Chelon saliens (Risso, 1810) & $x$ & \\
\hline & Mugil cephalus Linnaeus, 1758 & $x$ & \\
\hline Atherinidae & Atherina boyeri Risso, 1810 & $x$ & \\
\hline \multirow[t]{3}{*}{ Aphaniidae } & Aphanius apodus (Gervais, 1853) & $x$ & \\
\hline & Aphanius fasciatus (Valenciennes, 1821) & $x$ & \\
\hline & Aphanius saourensis Blanco, Hrbek \& Doadrio, 2006 & $x$ & \\
\hline Poeciliidae & Gambusia holbrooki Girard, 1859 & & $x$ \\
\hline Syngnathidae & Syngnathus abaster Risso, 1827 & $x$ & \\
\hline Moronidae & Dicentrarchus labrax (Linnaeus, 1758) & $x$ & \\
\hline \multirow[t]{3}{*}{ Centrarchidae } & Lepomis gibbosus (Linnaeus, 1758) & & $\mathrm{x}$ \\
\hline & Lepomis macrochirus Rafinesque, 1819 & & $\mathrm{x}$ \\
\hline & Micropterus salmoides (Lacepède, 1802) & & $x$ \\
\hline \multirow[t]{6}{*}{ Cichlidae } & Coptodon polycentra (Günther, 1862) & $x$ & \\
\hline & Coptodon zillii (Gervais, 1848) & $x$ & \\
\hline & Haplochromis desfontainii (Lacepède, 1802) & $x$ & \\
\hline & Hemichromis guttatus Günther, 1862 & $x$ & \\
\hline & Oreochromis aureus (Steindachner, 1864) & $x$ & \\
\hline & Oreochromis niloticus (Linnaeus, 1758) & & $x$ \\
\hline \multirow[t]{2}{*}{ Percidae } & Perca fluviatilis Linnaeus, 1758 & & $x$ \\
\hline & Sander lucioperca (Linnaeus, 1758) & & $x$ \\
\hline \multirow[t]{2}{*}{ Blenniidae } & Salaria atlantica Doadrio, Perea \& Yahyaoui, 2011 & $x$ & \\
\hline & Salaria fluviatilis (Asso y del Rio, 1801) & $x$ & \\
\hline
\end{tabular}




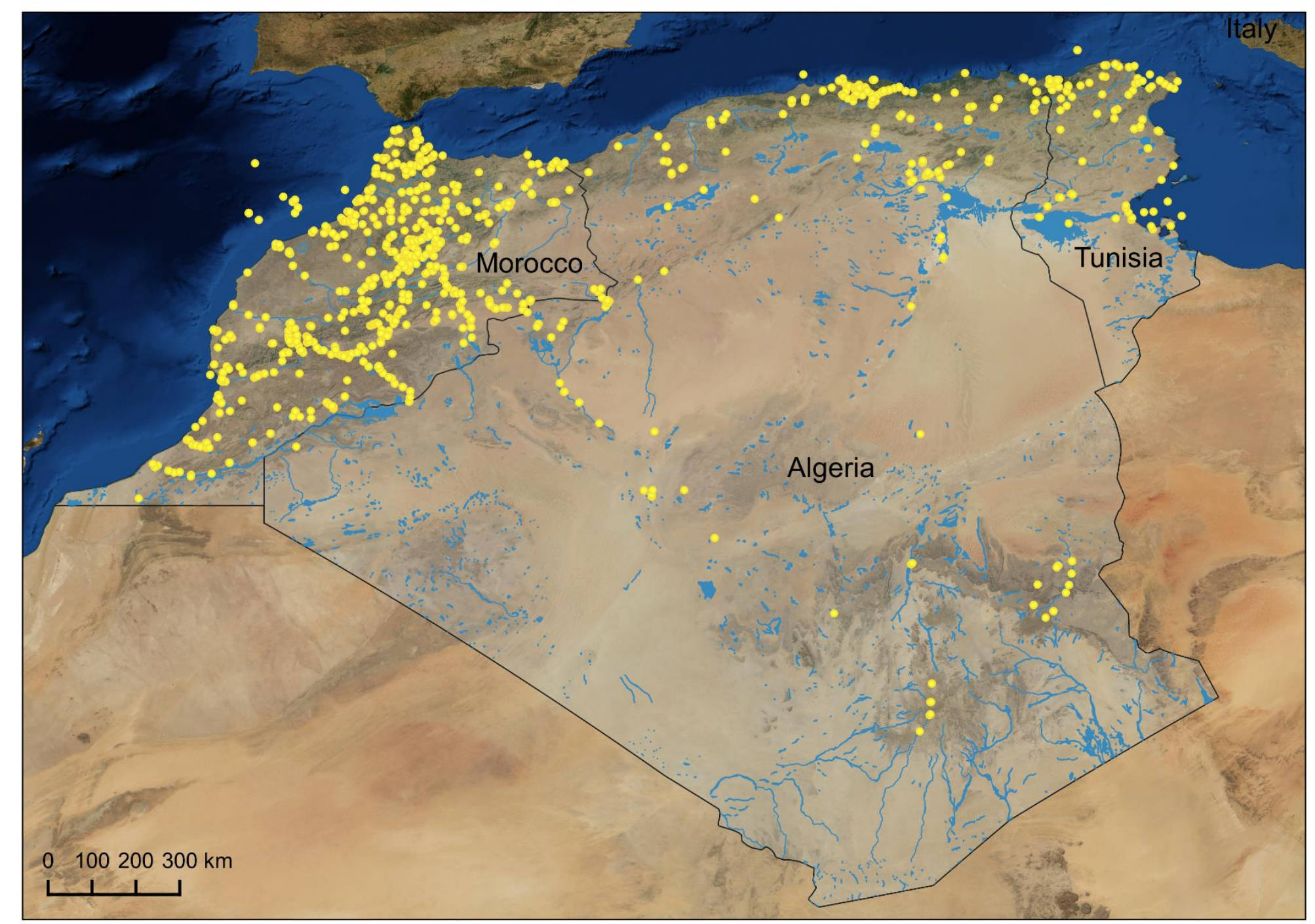

Figure 1: Overview map depicting Maghreb region and all 2050 site scale occurrence records collated during this study. 


\section{Family: Petromyzontidae}

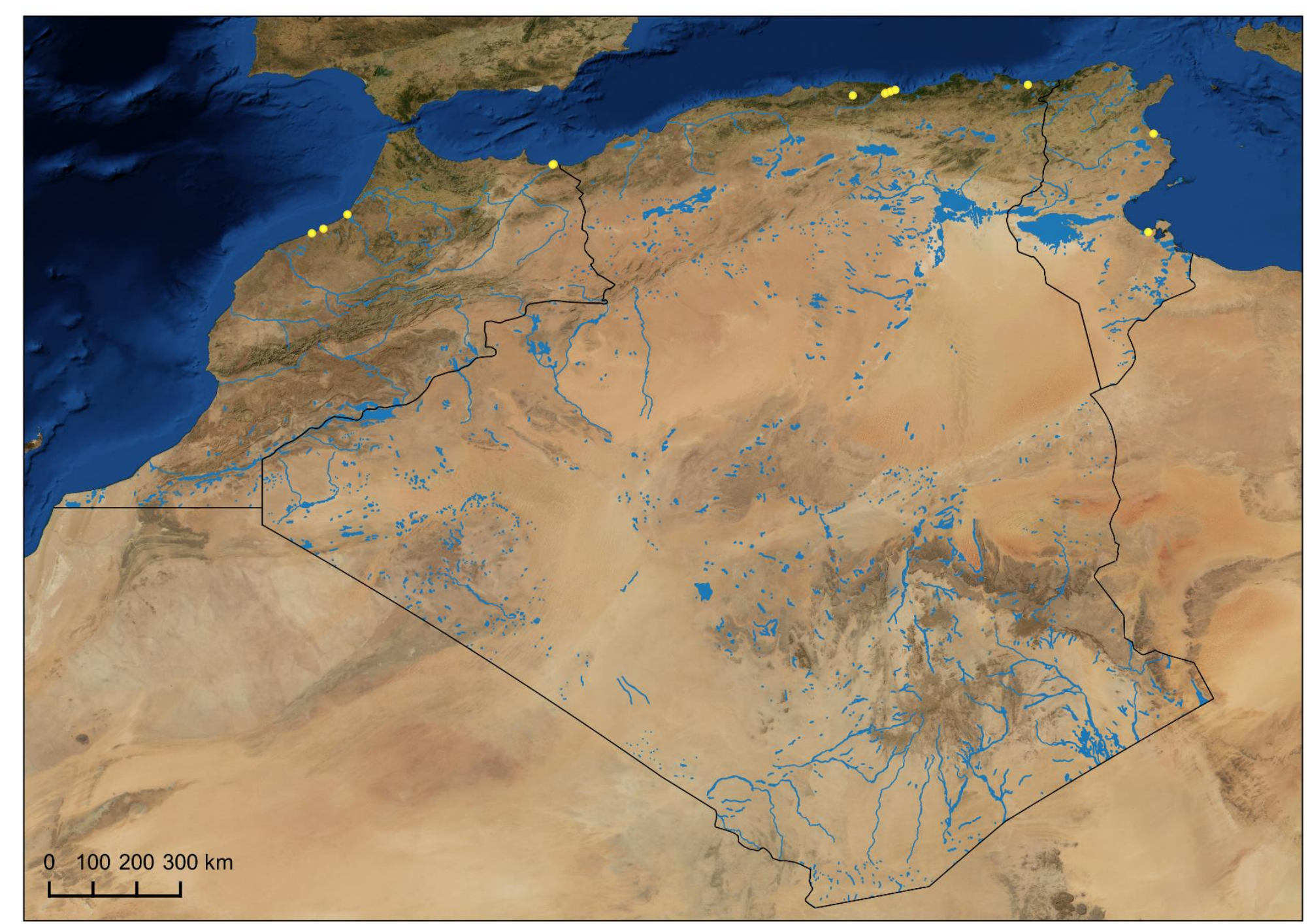

Figure 2: Petromyzon marinus. 


\section{Family: Acipenseridae}

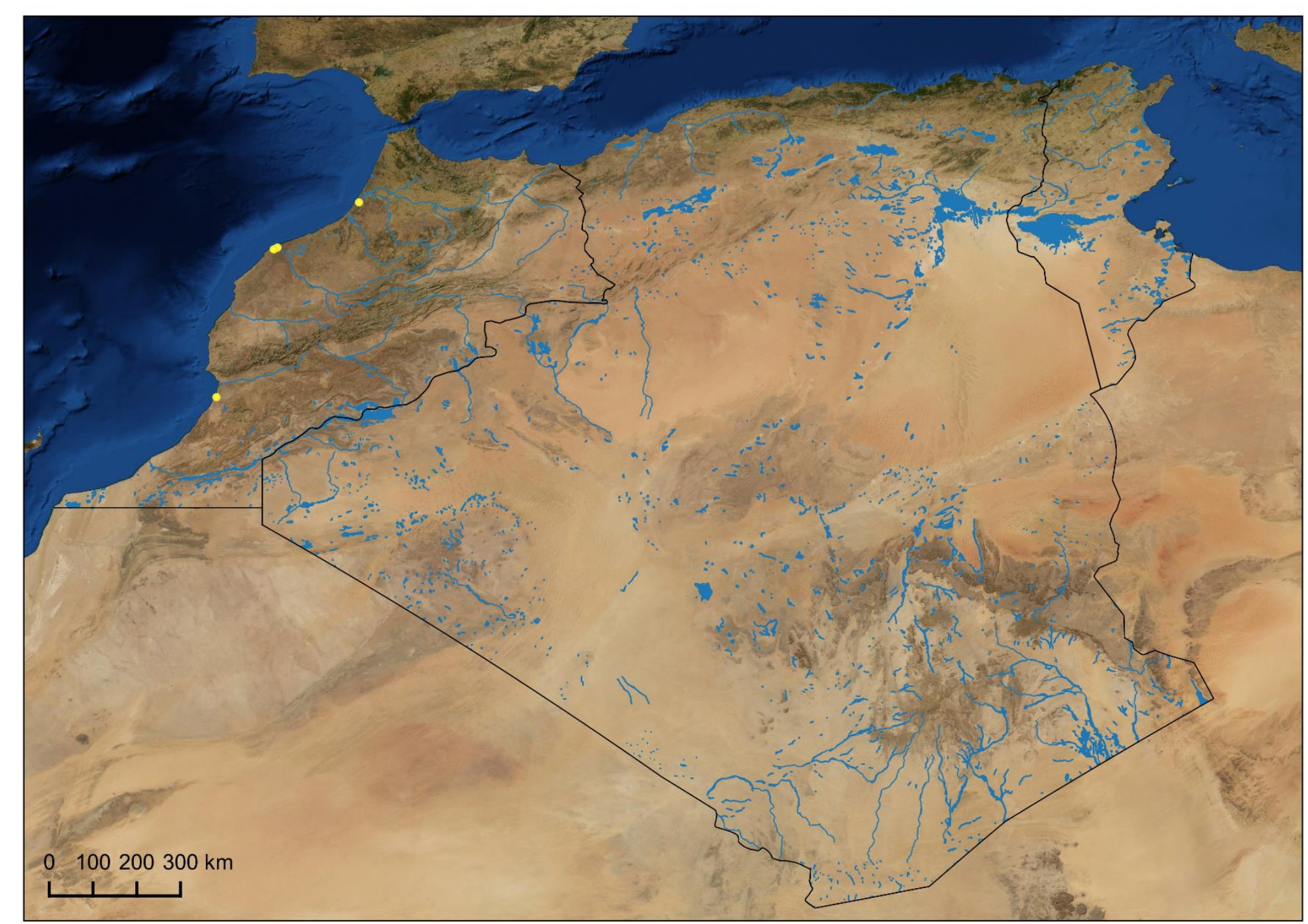

Figure 3: Acipenser sturio. Not reported from the region since the mid-1950s. 


\section{Family: Anguillidae}

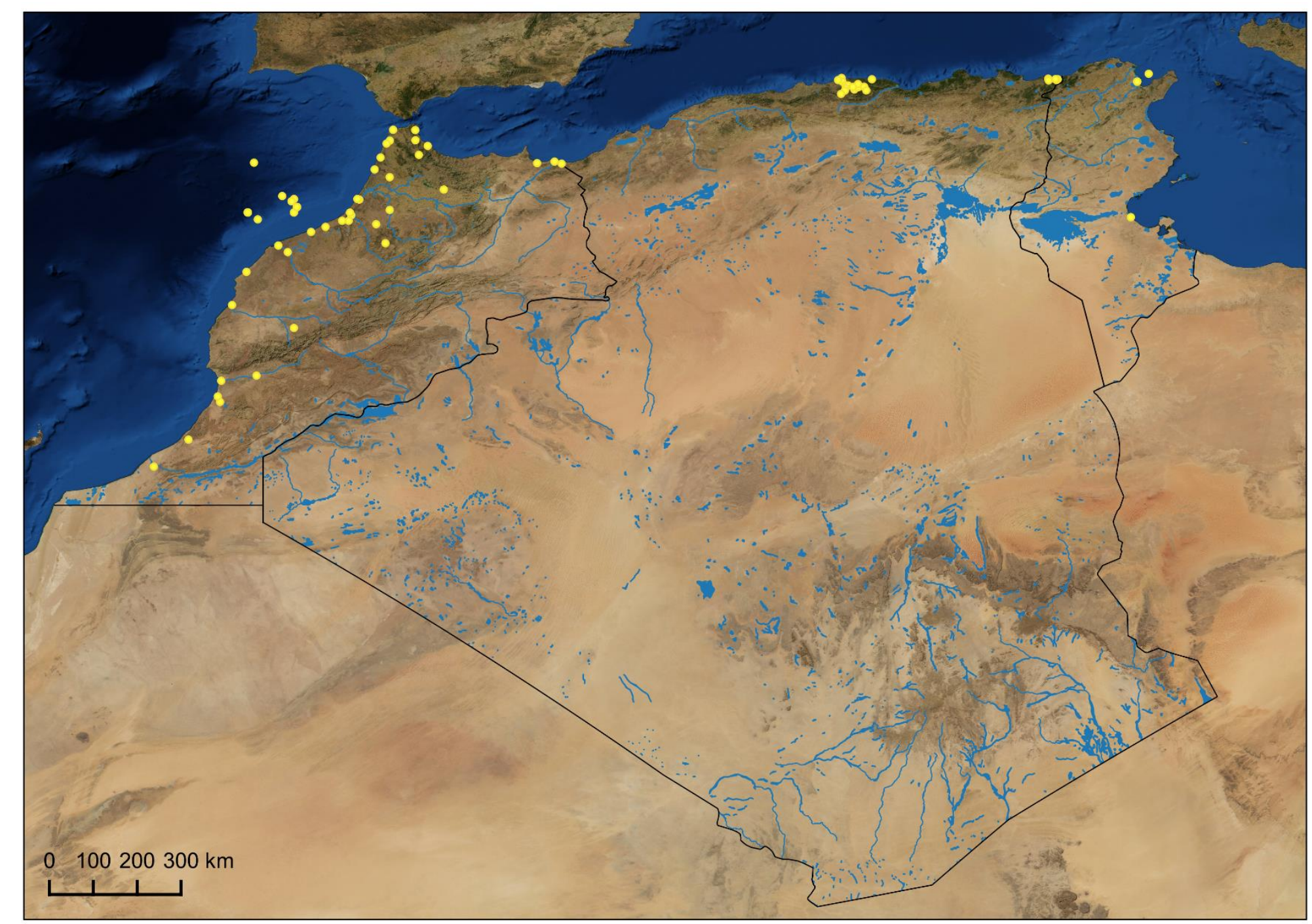

Figure 4: Anguilla anguilla. Expected to be present in all permanent coastal rivers and lakes. 


\section{Family: Clupeidae}

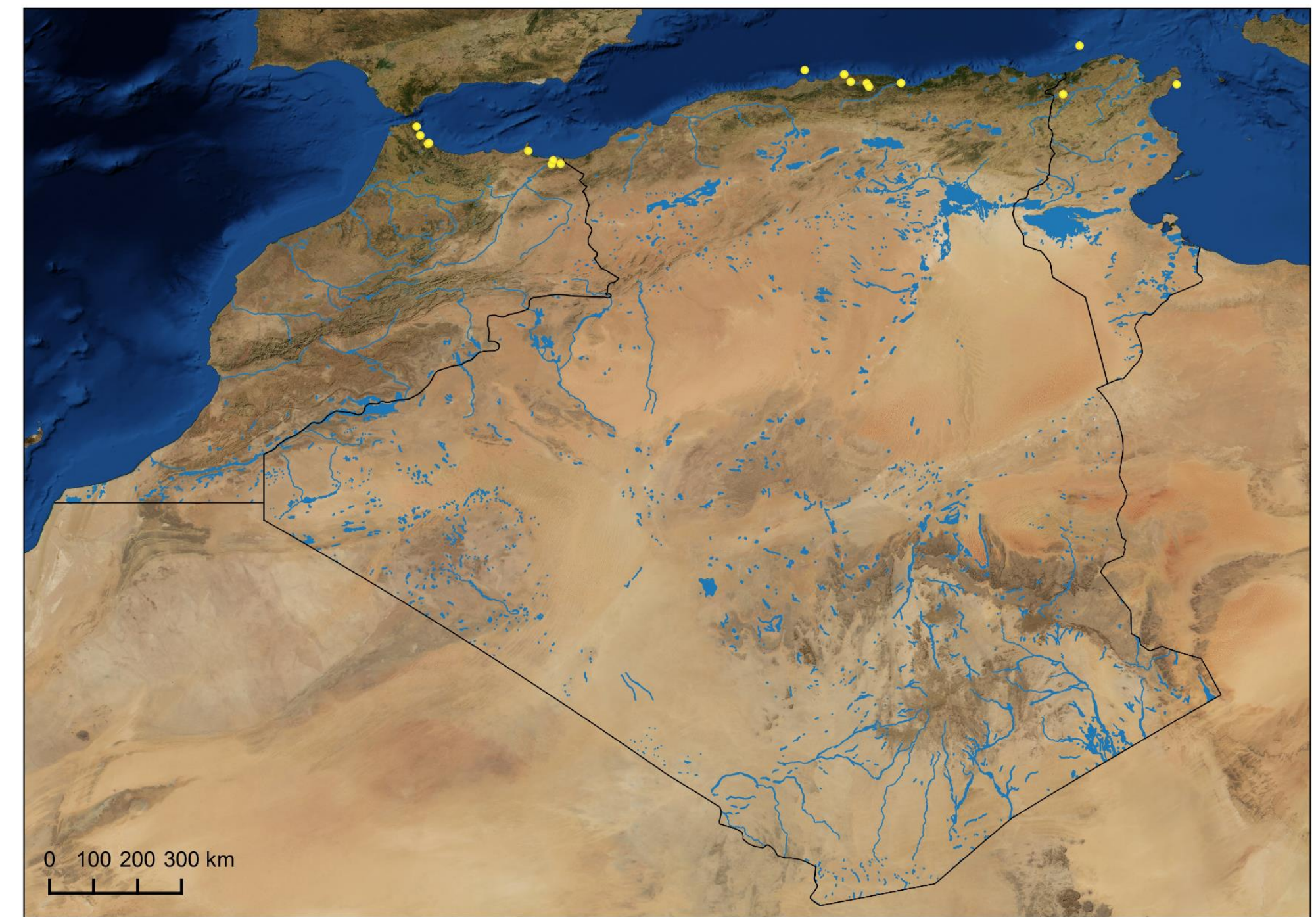

Figure 5: Alosa algeriensis. 


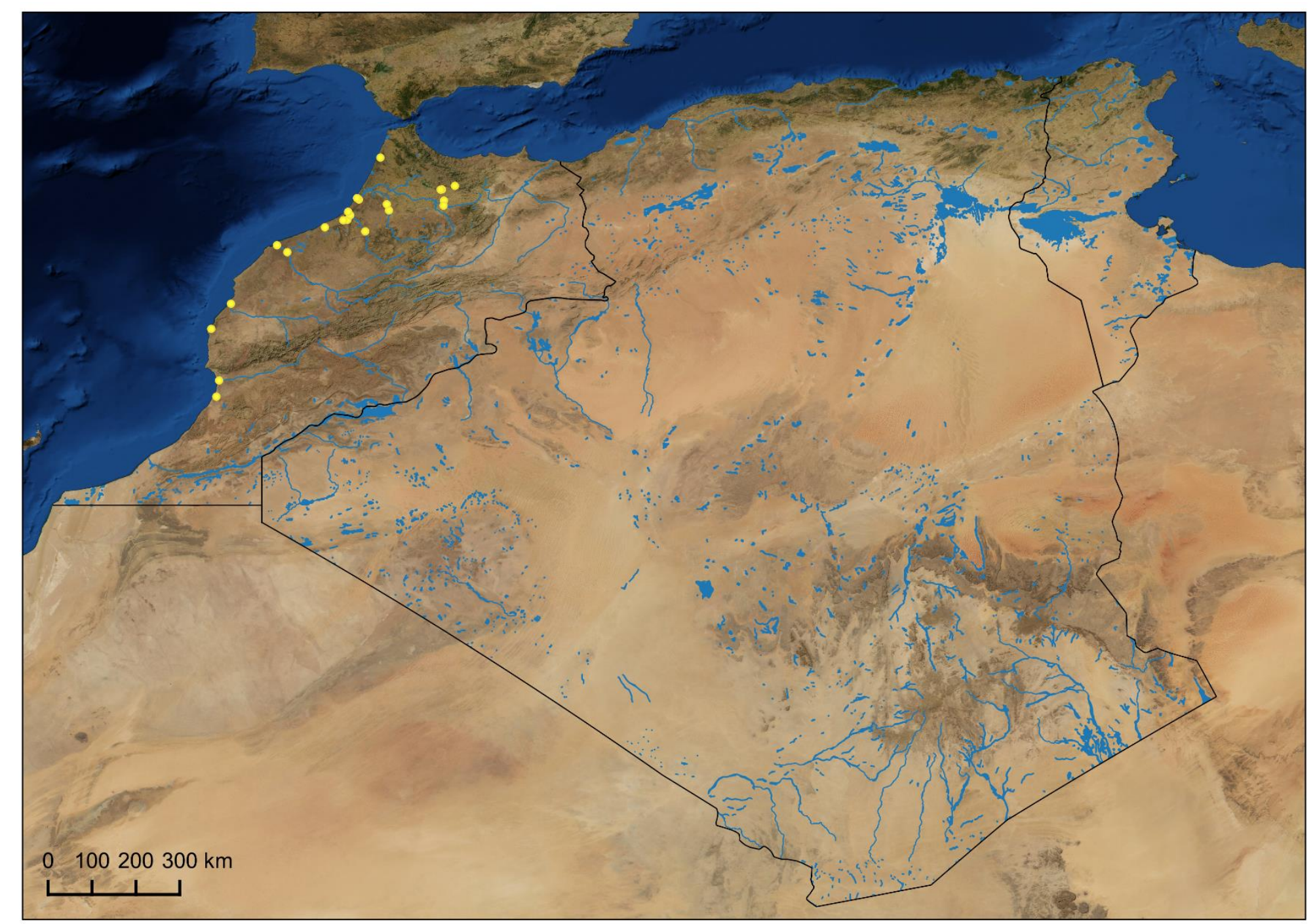

Figure 6: Alosa alosa. May no longer be present in the region but additional field research is required. 


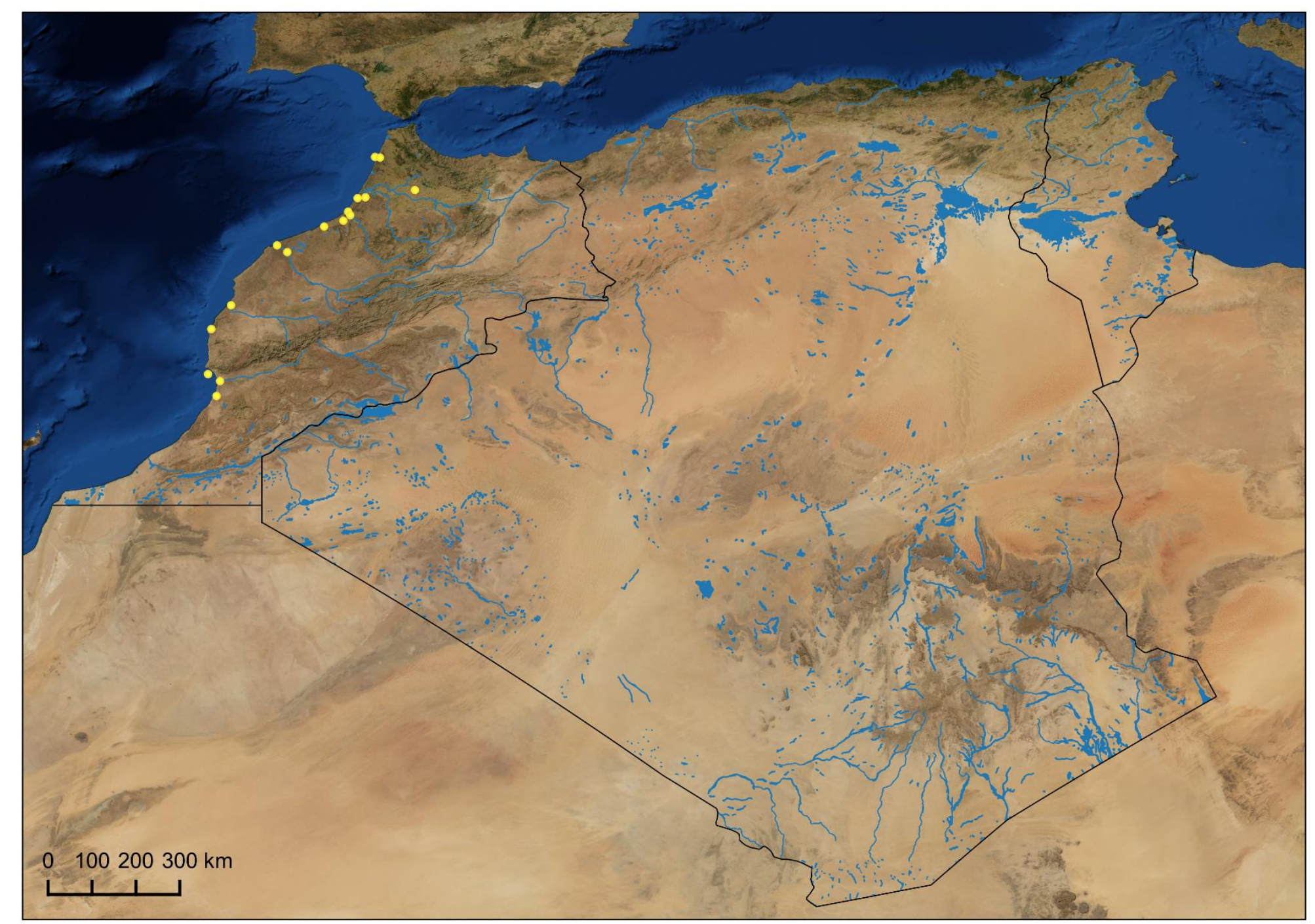

Figure 7: Alosa fallax. May no longer be present in the region but additional field research is required. 


\section{Family: Cyprinidae}

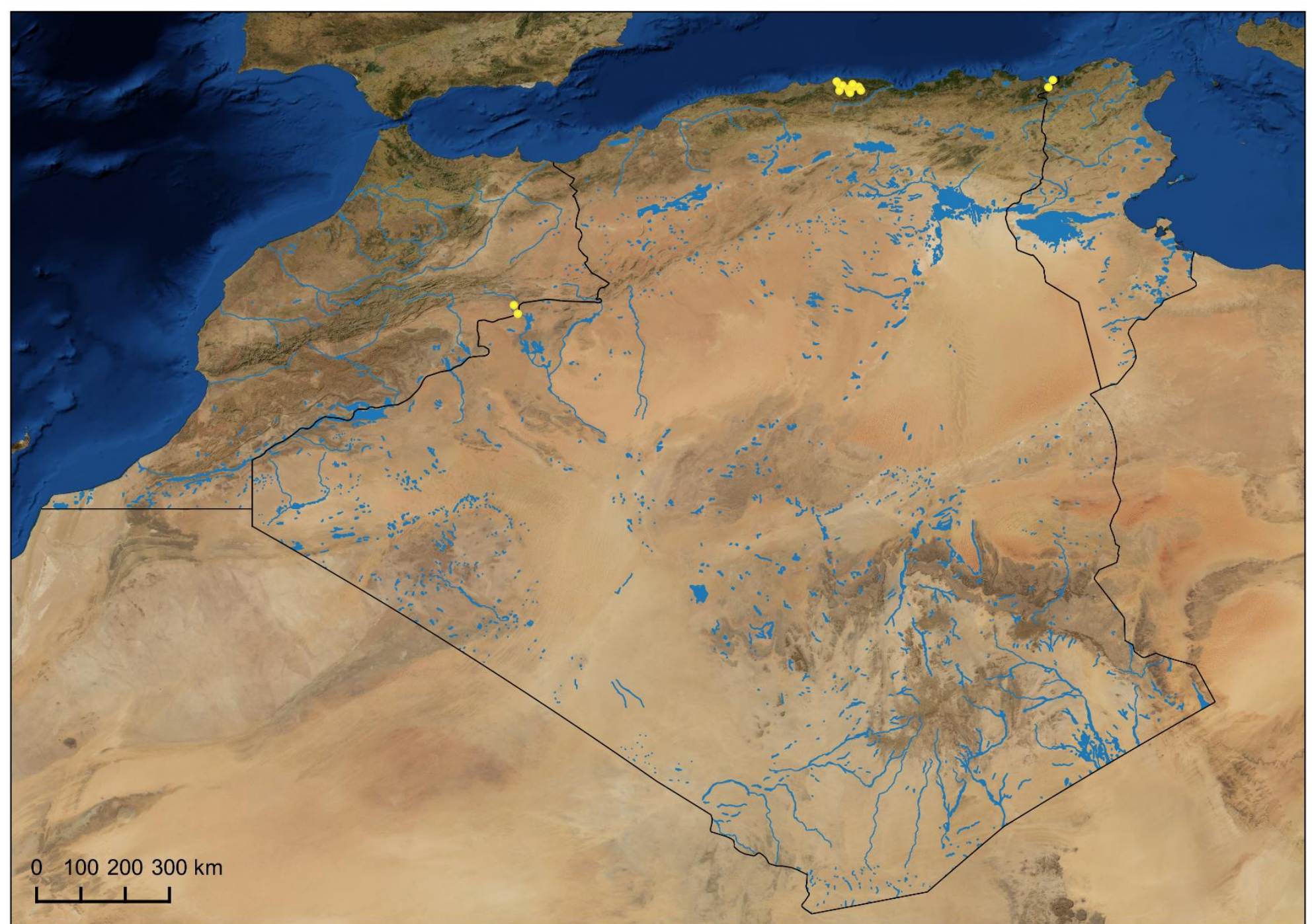

Figure 8: Carassius auratus. Known to be widespread in the region but few precise site-scale records exist. 


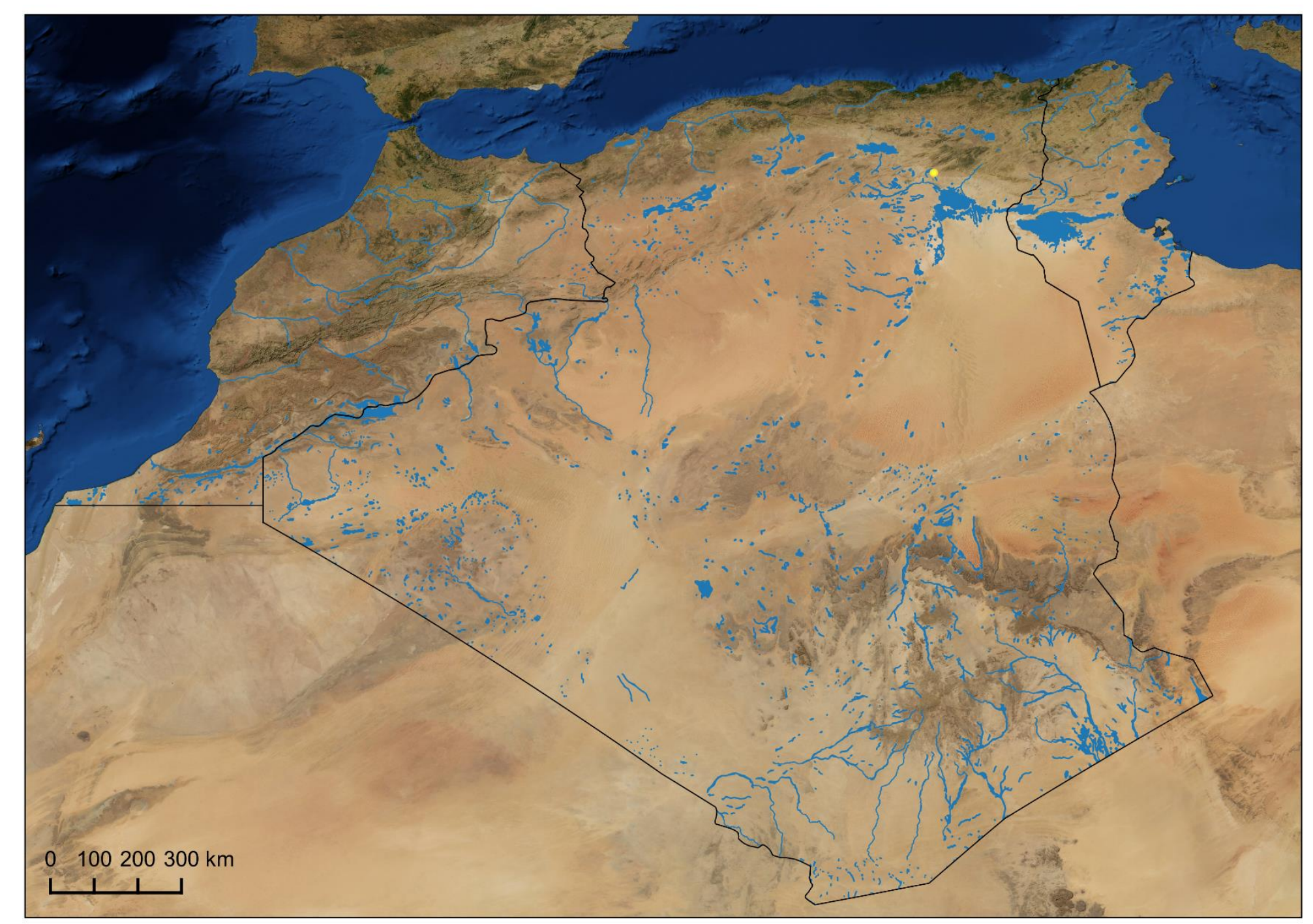

Figure 9: Carassius langsdorfii. 


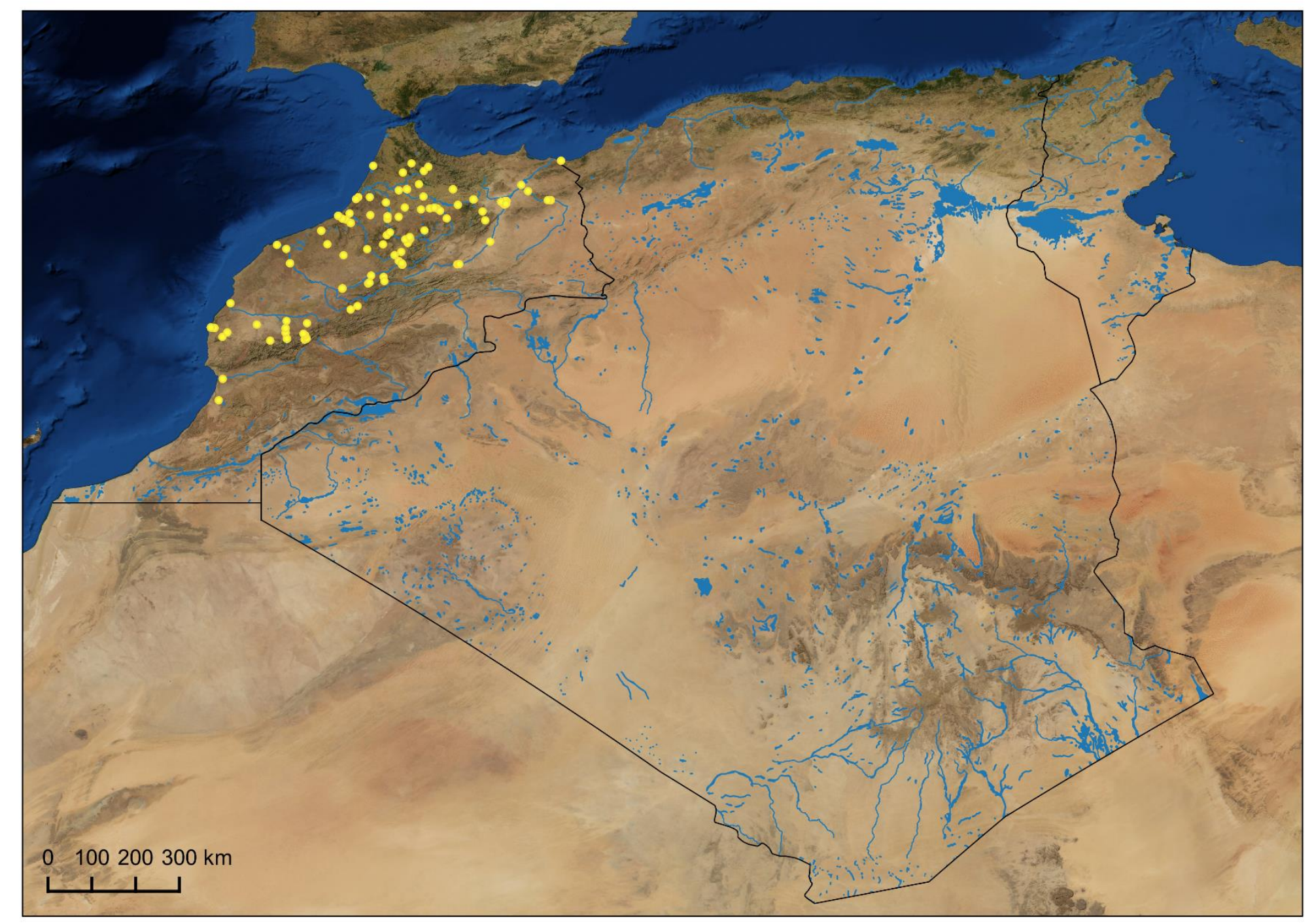

Figure 10: Carasobarbus fritschii. 


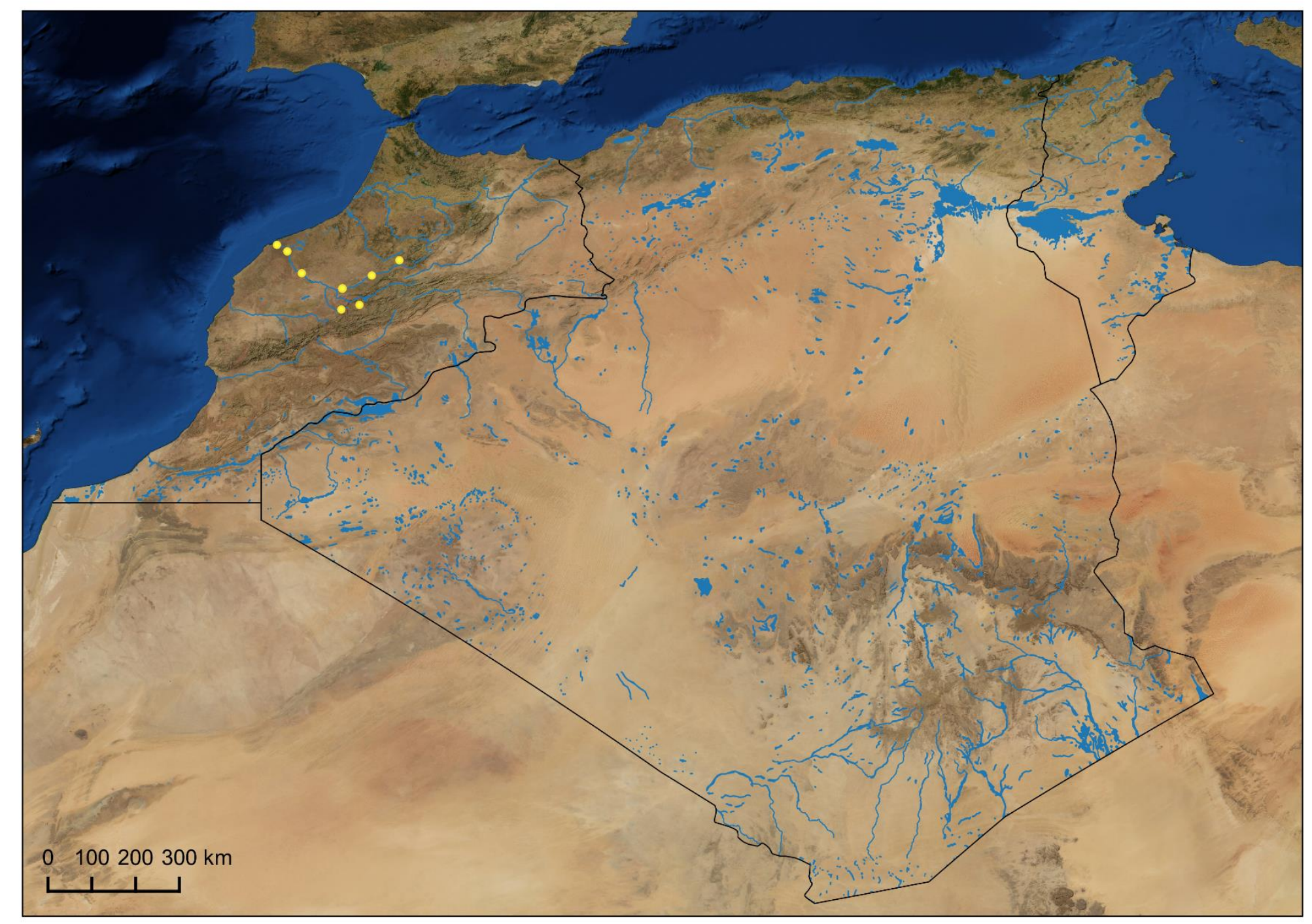

Figure 11: Carasobarbus harterti. Possibly extirpated from several former locations, but further field research is needed. 


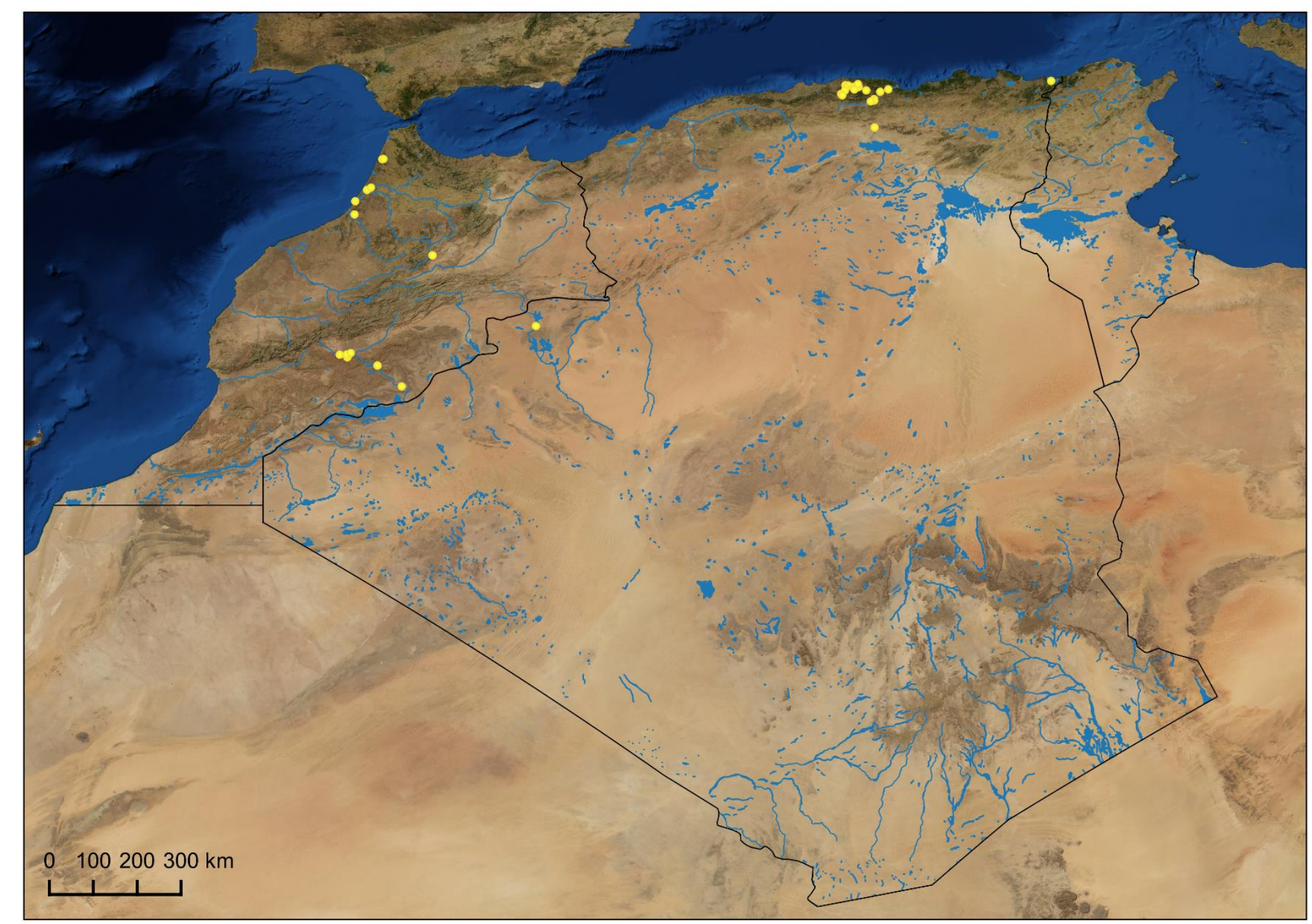

Figure 12: Cyprinus carpio. Known to be widespread in the region but few precise site-scale records exist. 


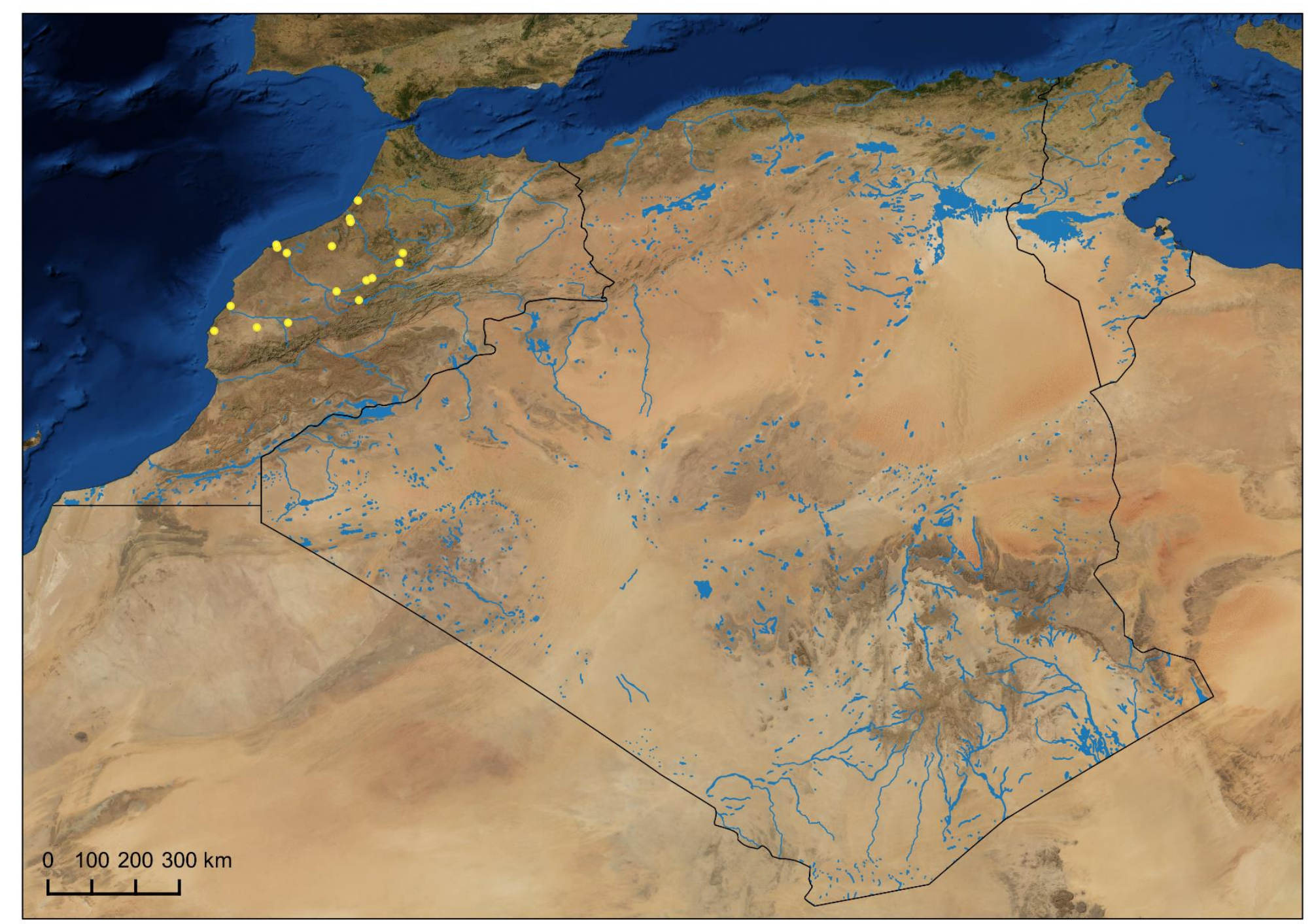

Figure 13: Labeobarbus reinii. All records are at least 18 years old. 


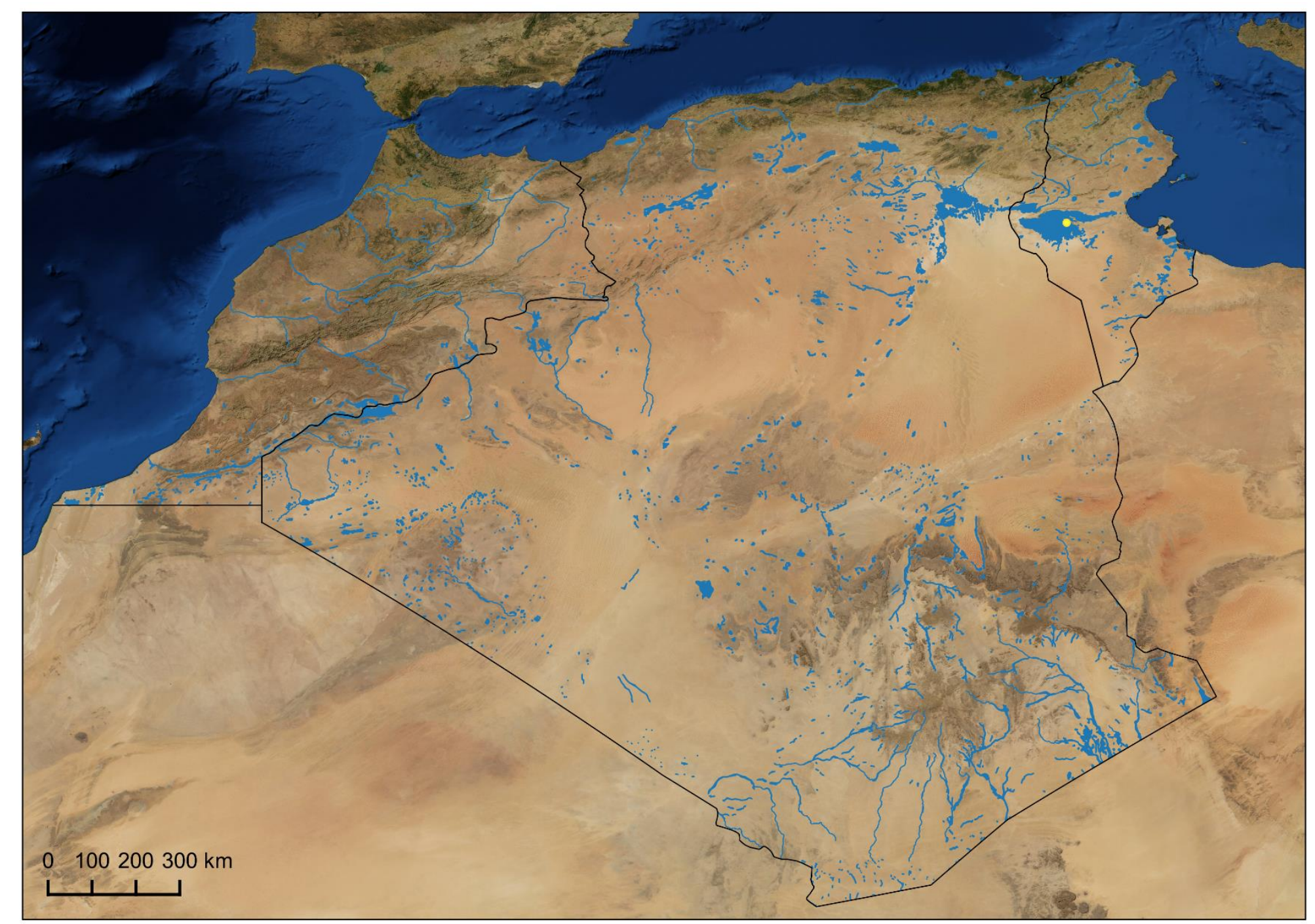

Figure 14: Luciobarbus antinorii. Extinct since the late 1980s. 


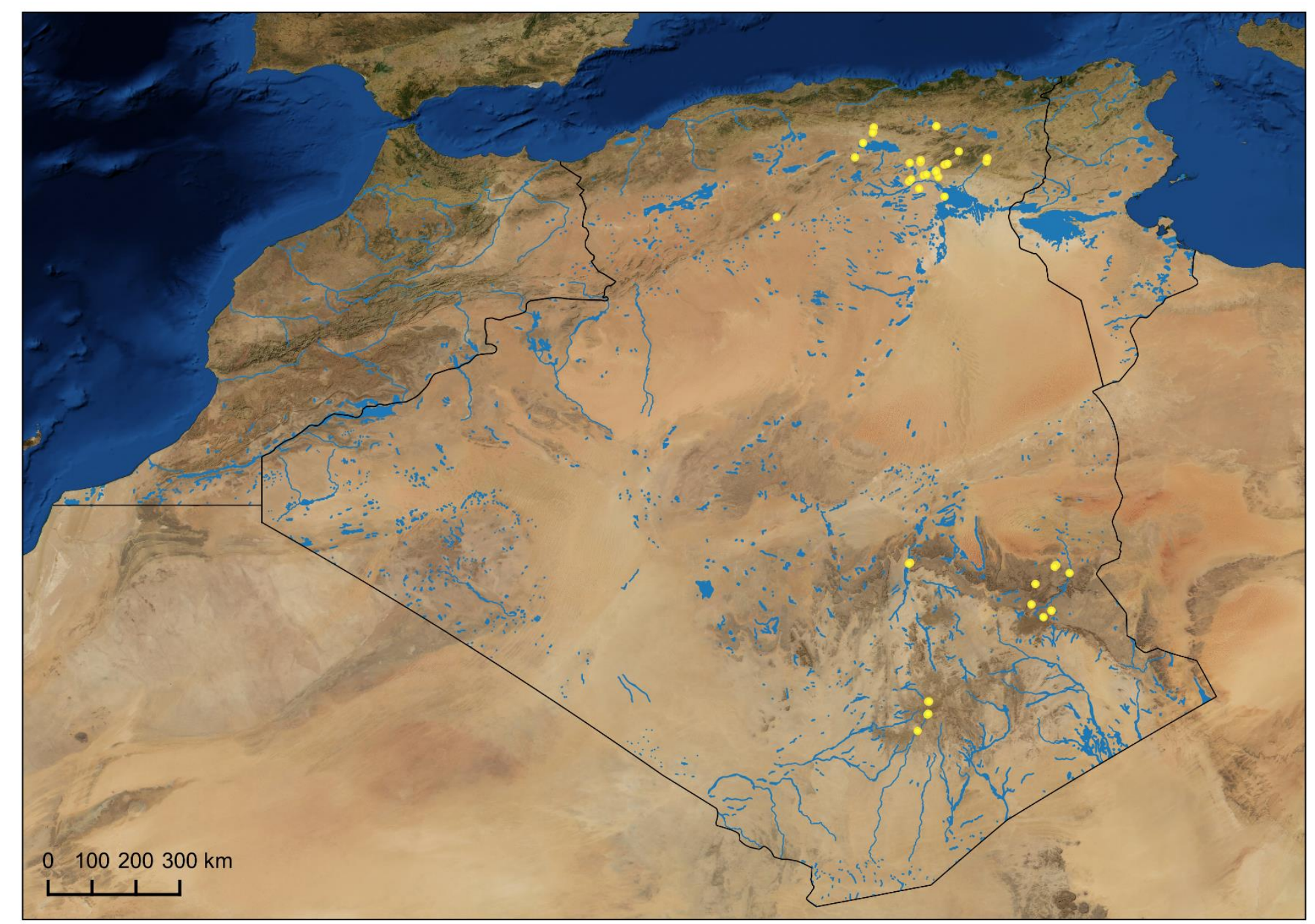

Figure 15: Luciobarbus biscarensis. 


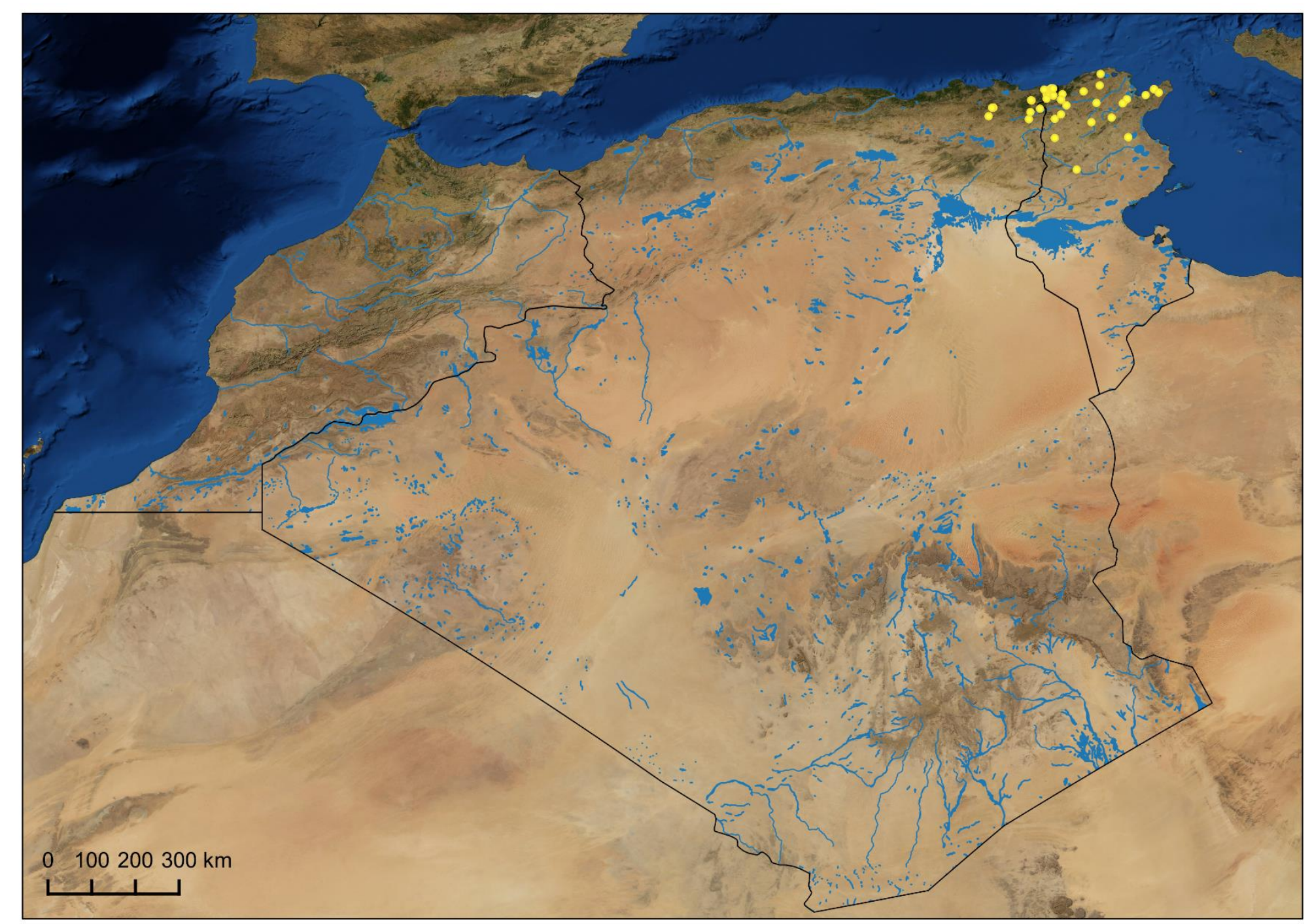

Figure 16: Luciobarbus callensis. 


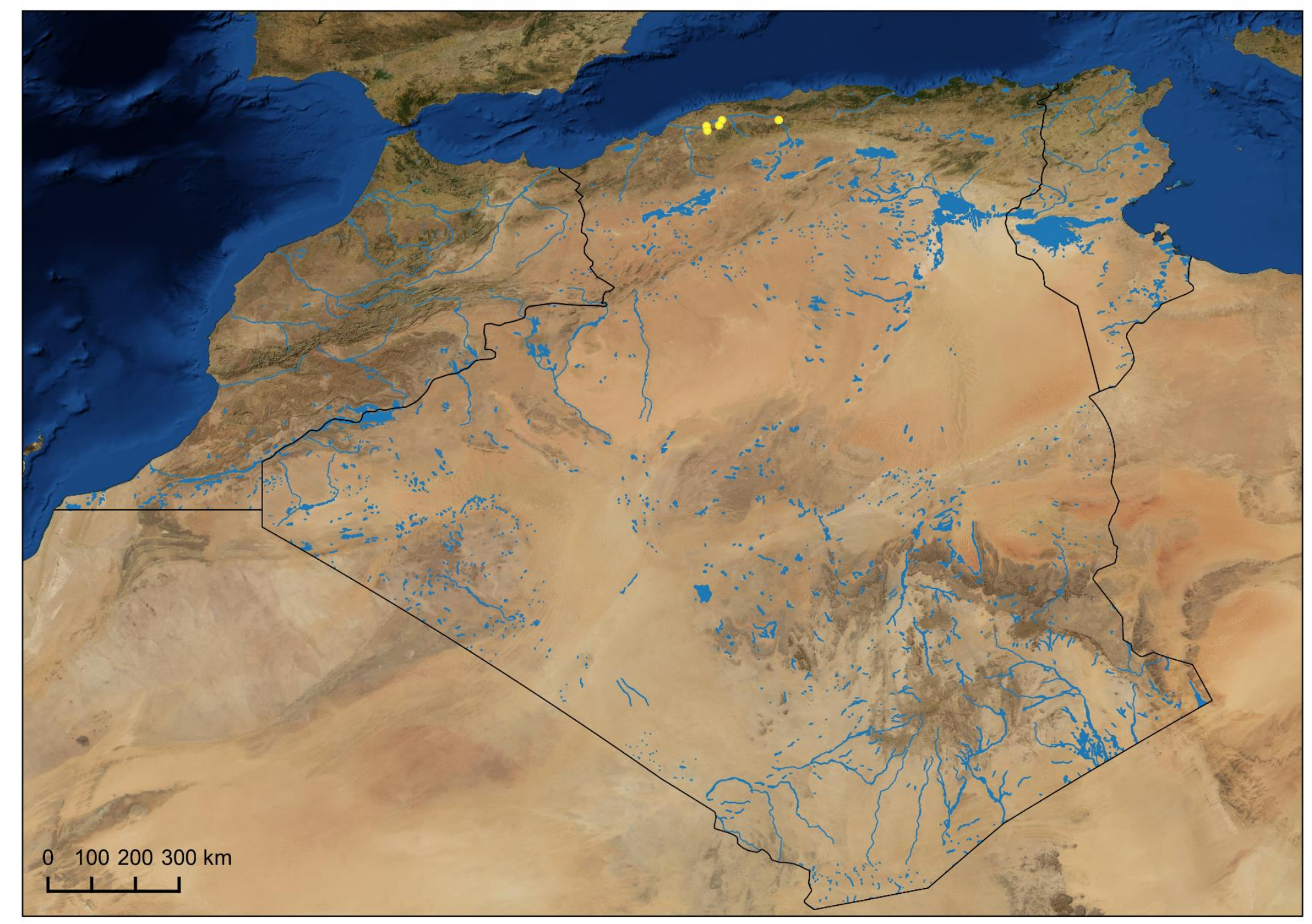

Figure 17: Luciobarbus chelifensis. 


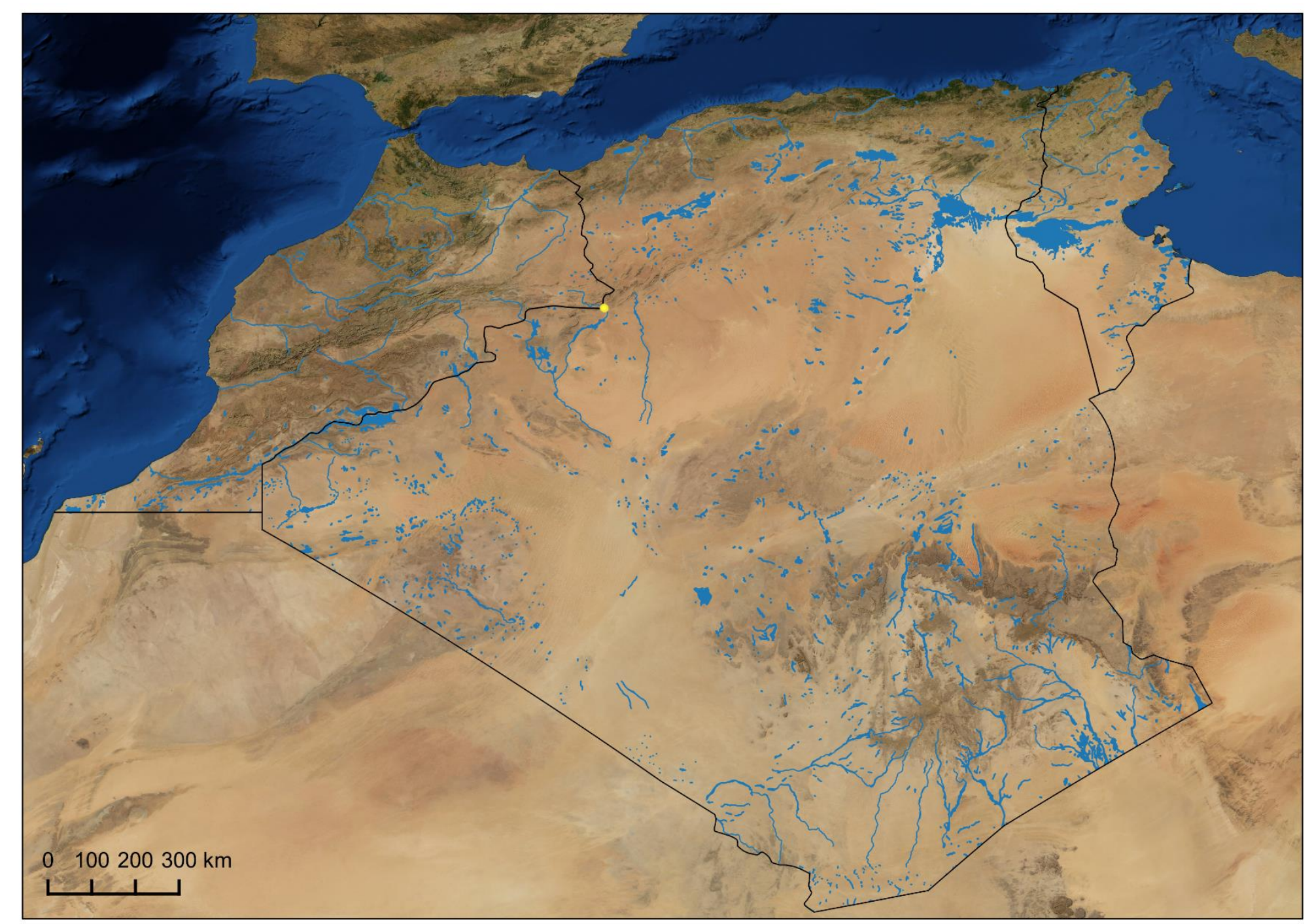

Figure 18: Luciobarbus figuigensis. 


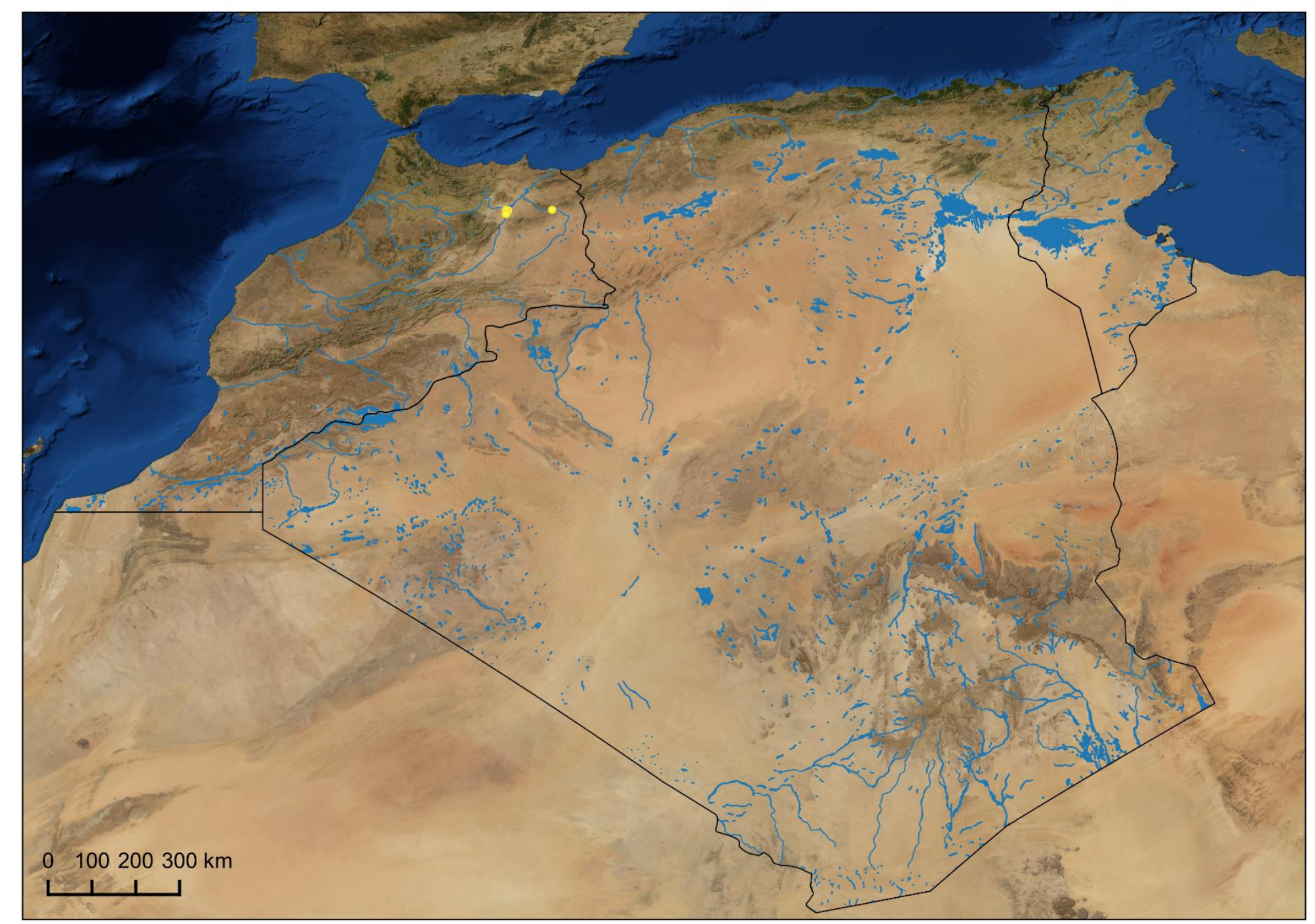

Figure 19: Luciobarbus guercifensis. 


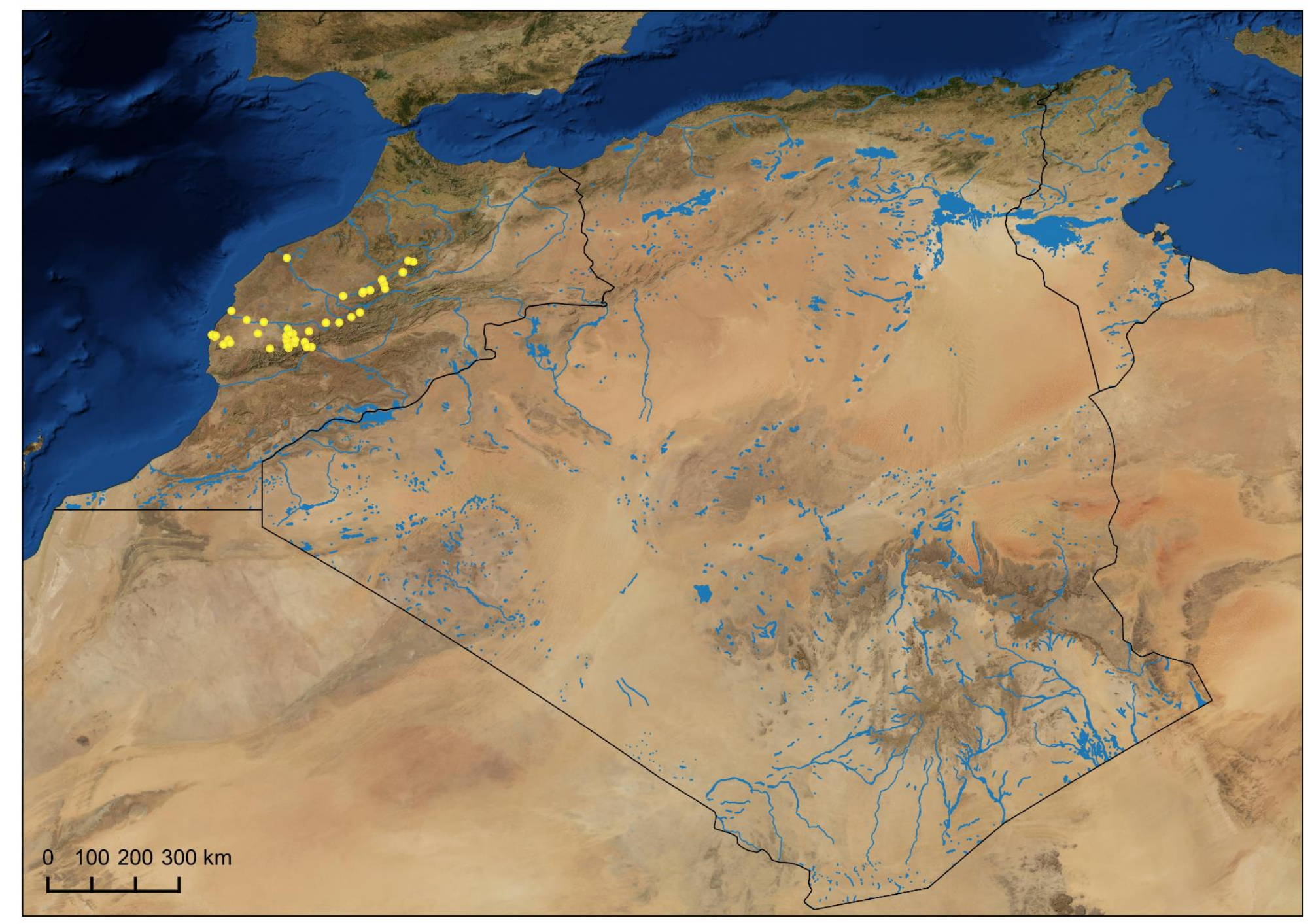

Figure 20: Luciobarbus ksibi. 


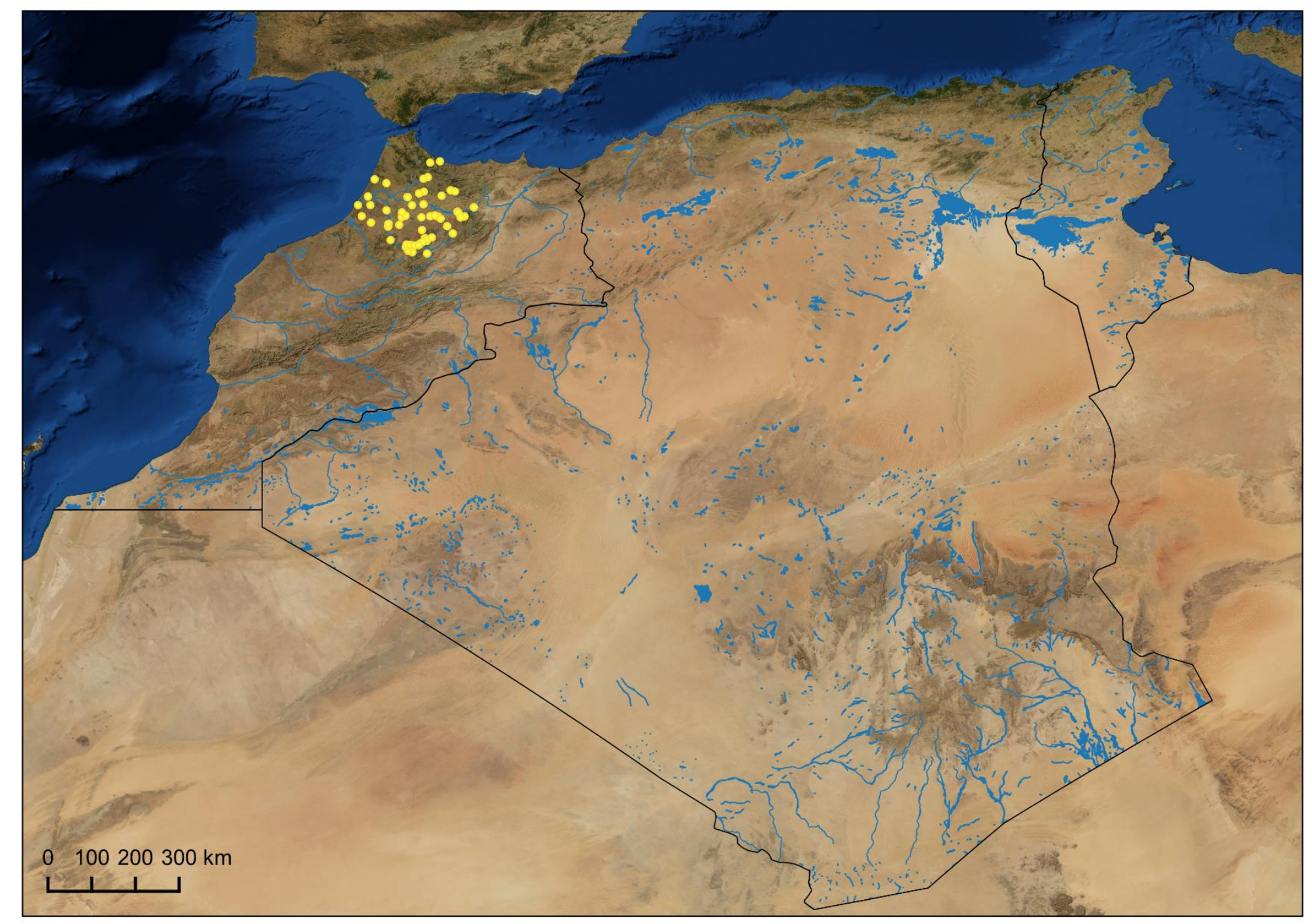

Figure 21: Luciobarbus labiosa. 


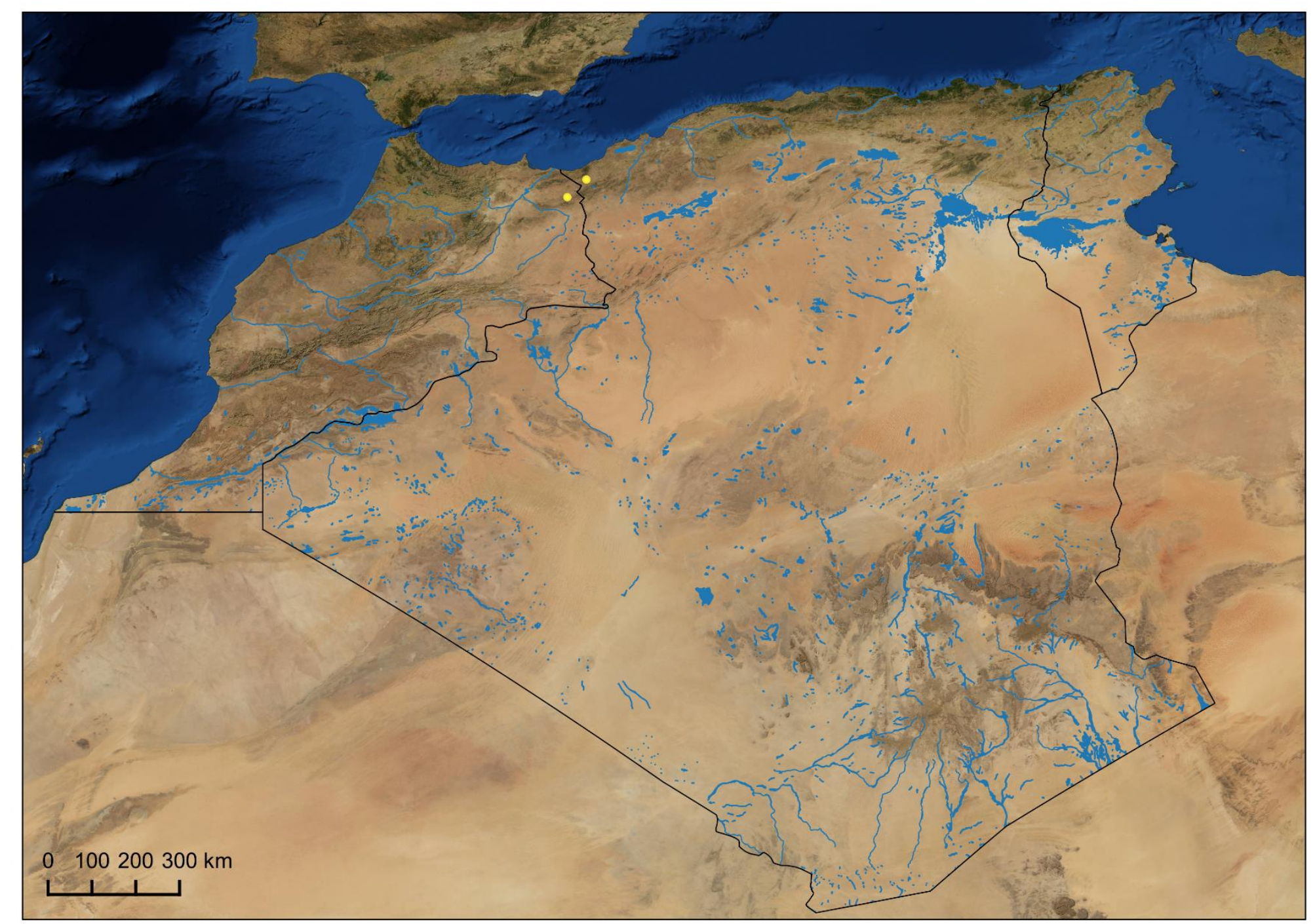

Figure 22: Luciobarbus lanigarensis. 


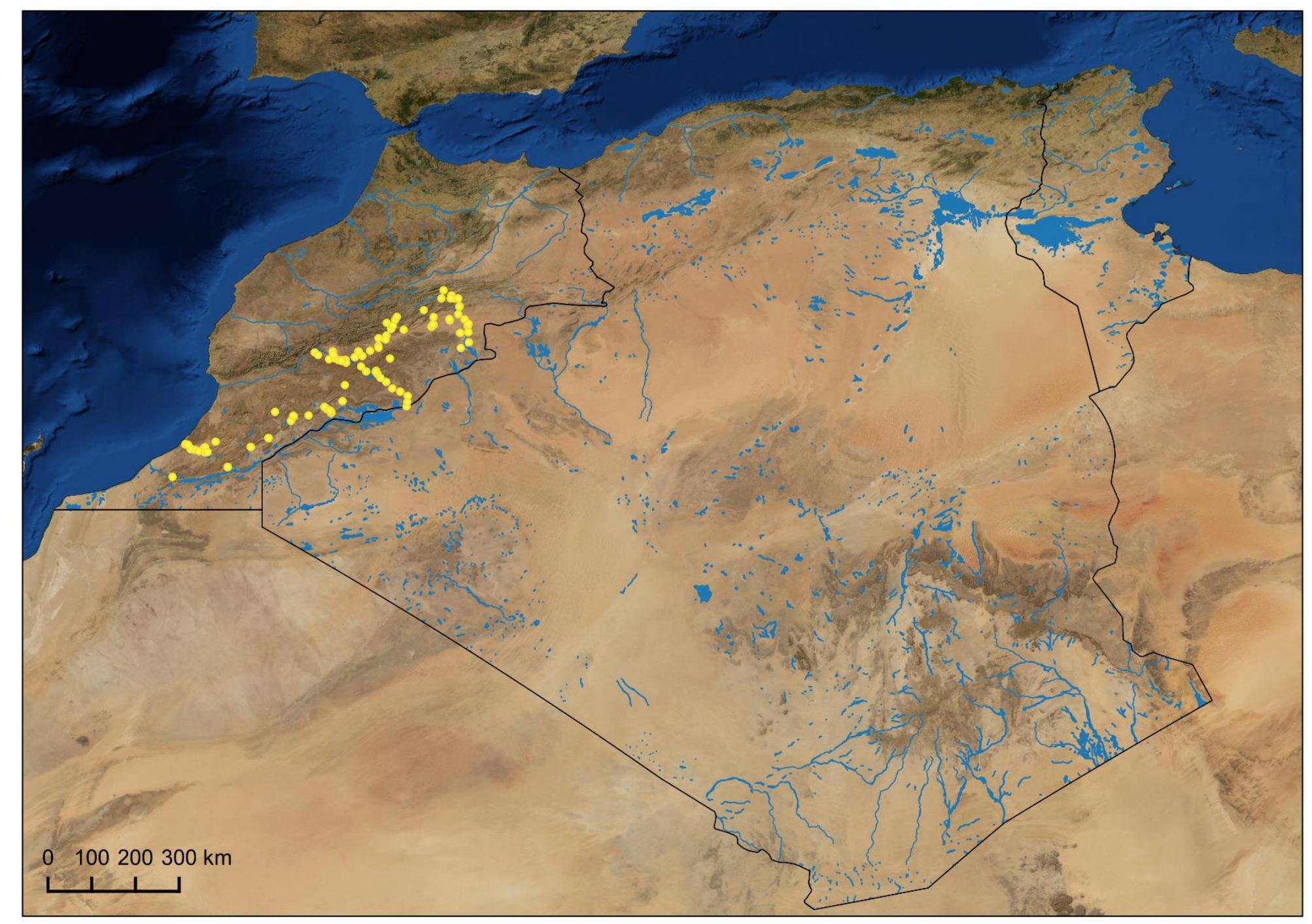

Figure 23: Luciobarbus lepineyi. 


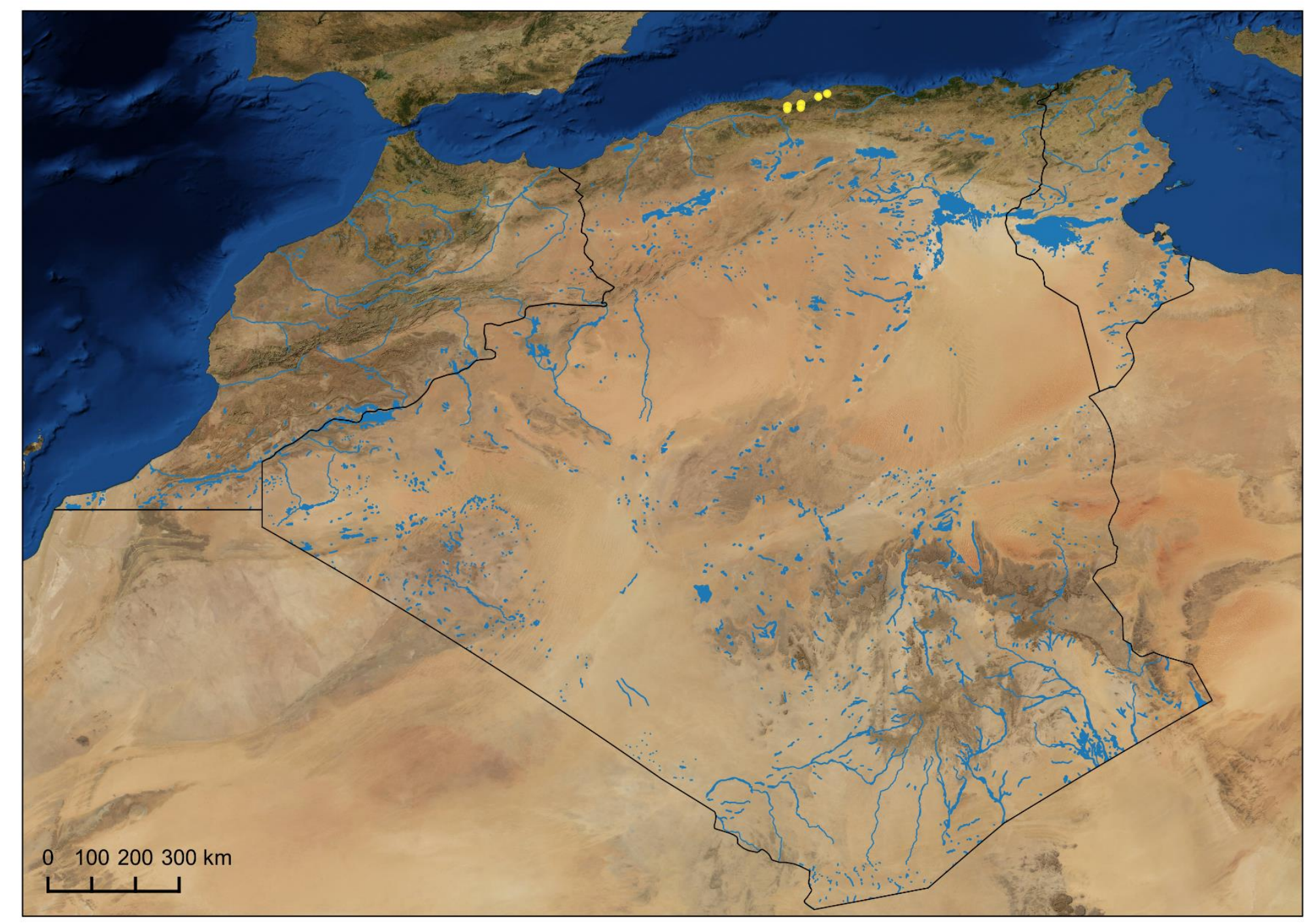

Figure 24: Luciobarbus leptopogon. 


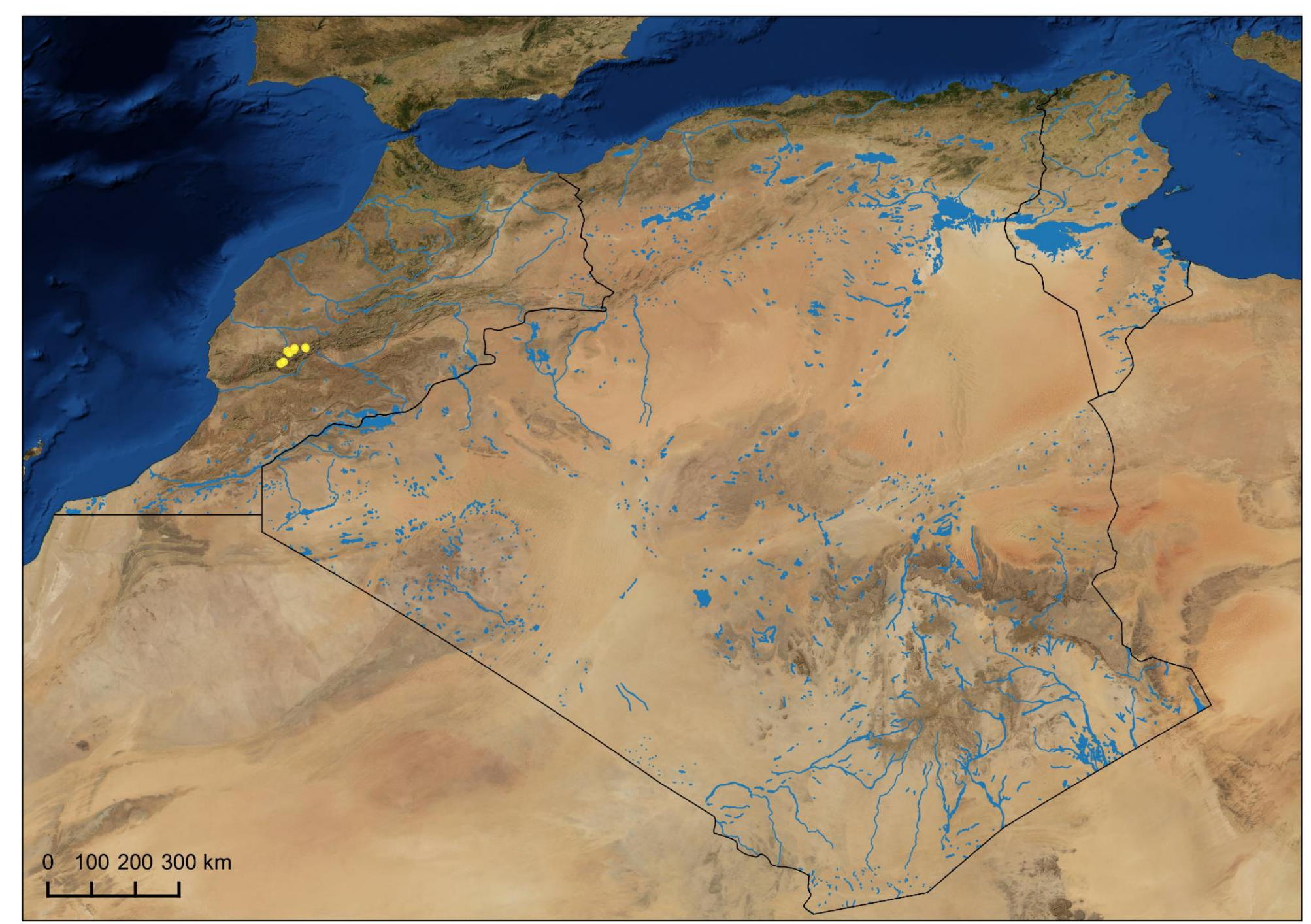

Figure 25: Luciobarbus magniatlantis. 


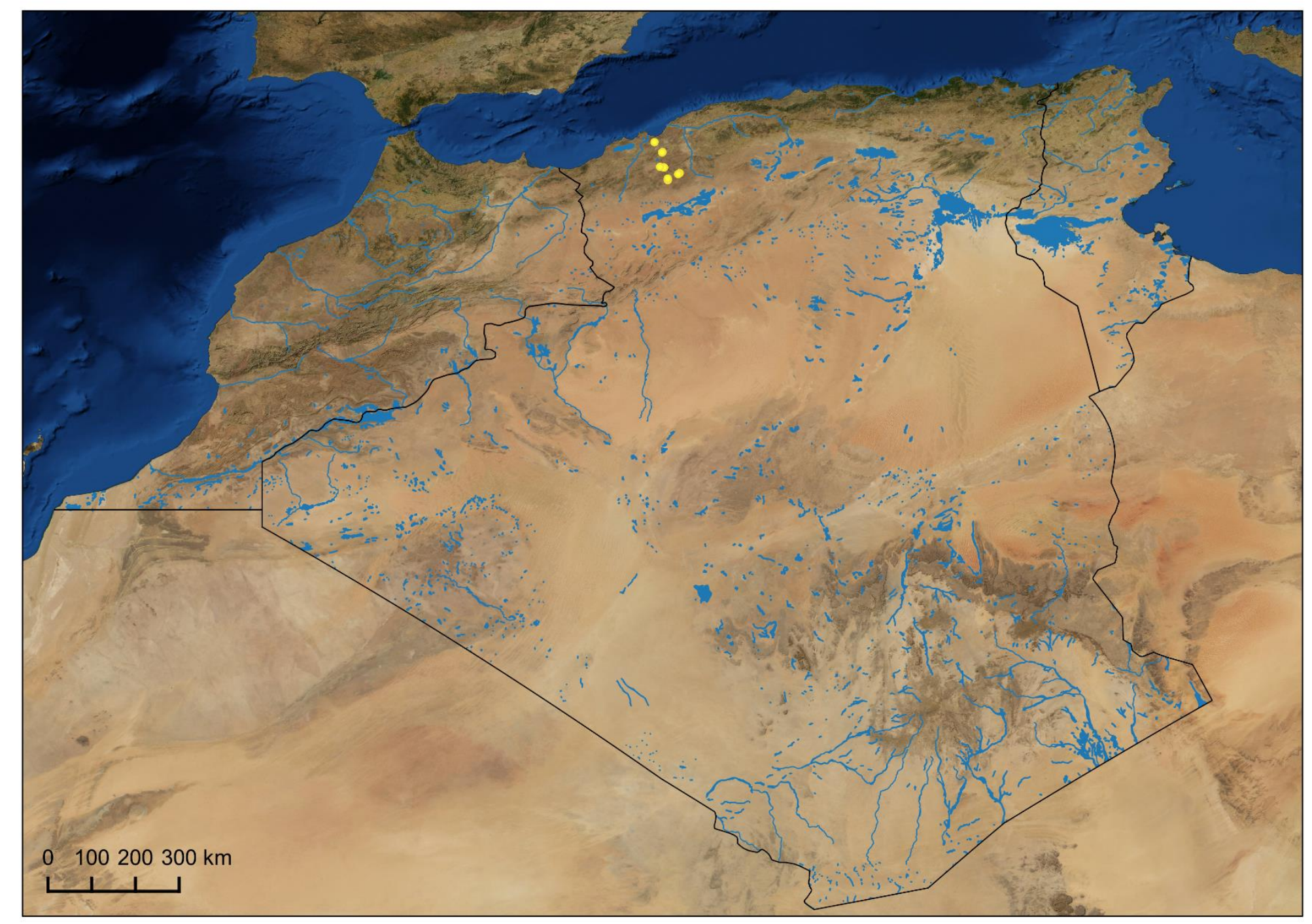

Figure 26: Luciobarbus mascarensis. 


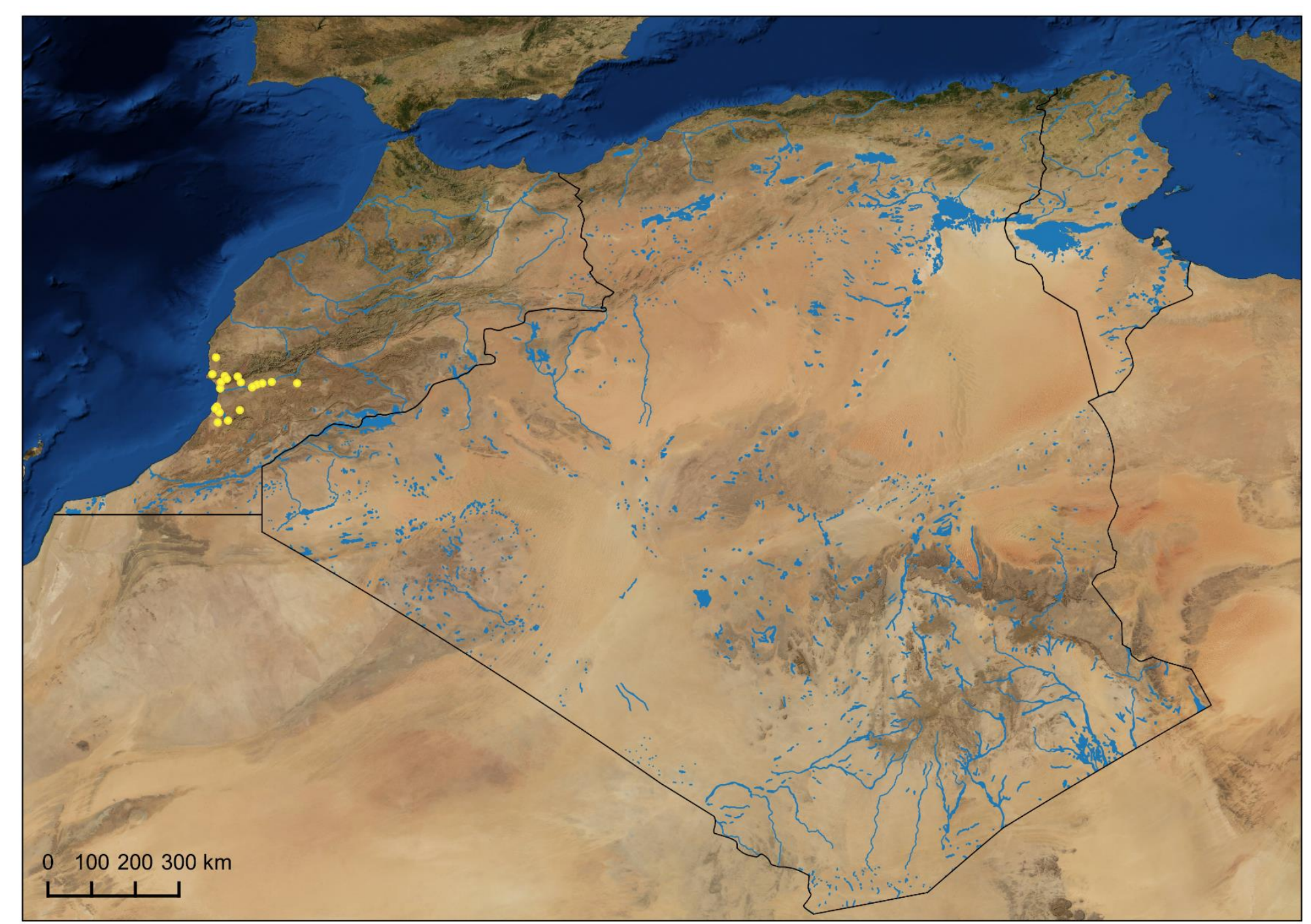

Figure 27: Luciobarbus massaensis. 


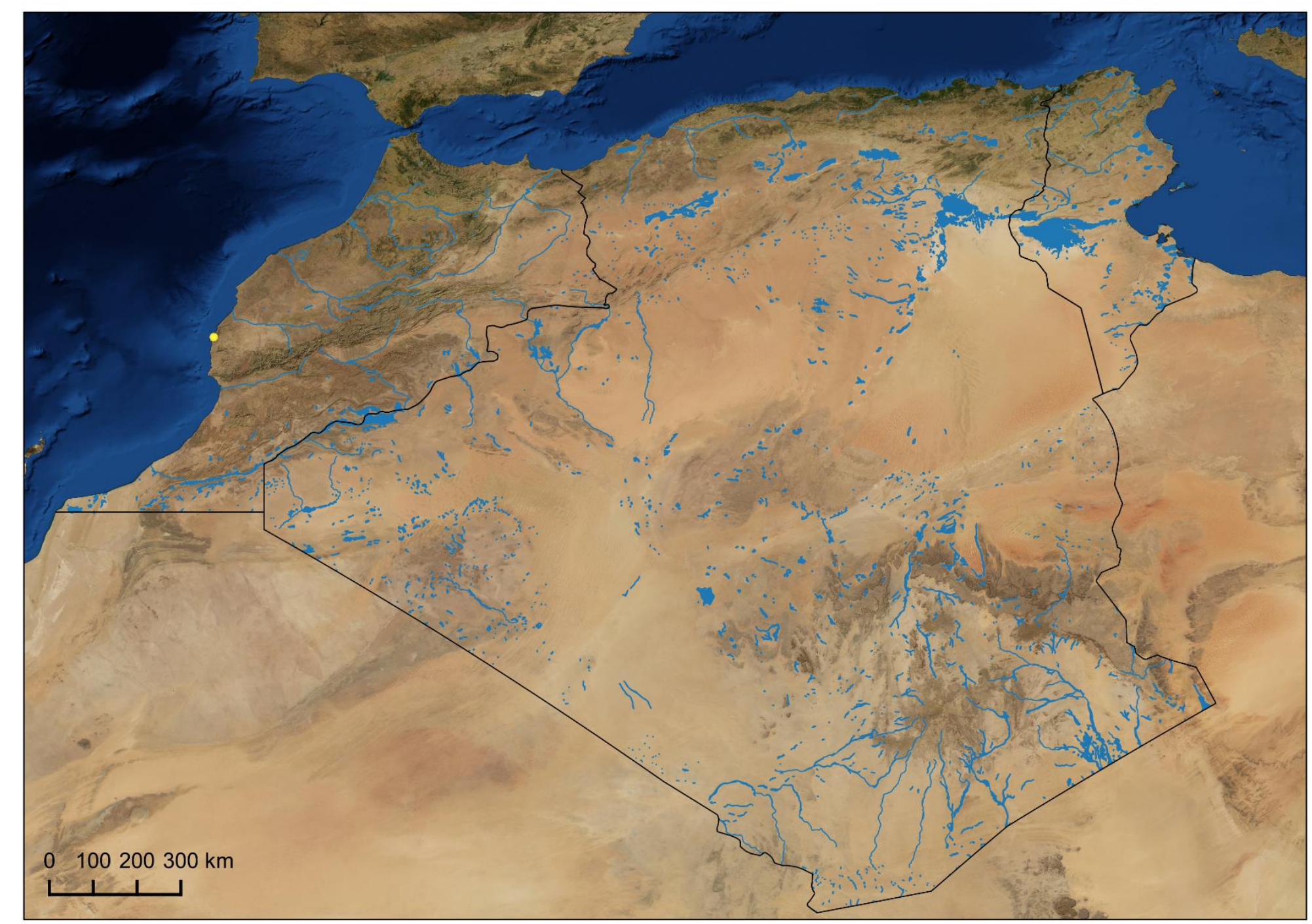

Figure 28: Luciobarbus nasus. Known only from the type series. 


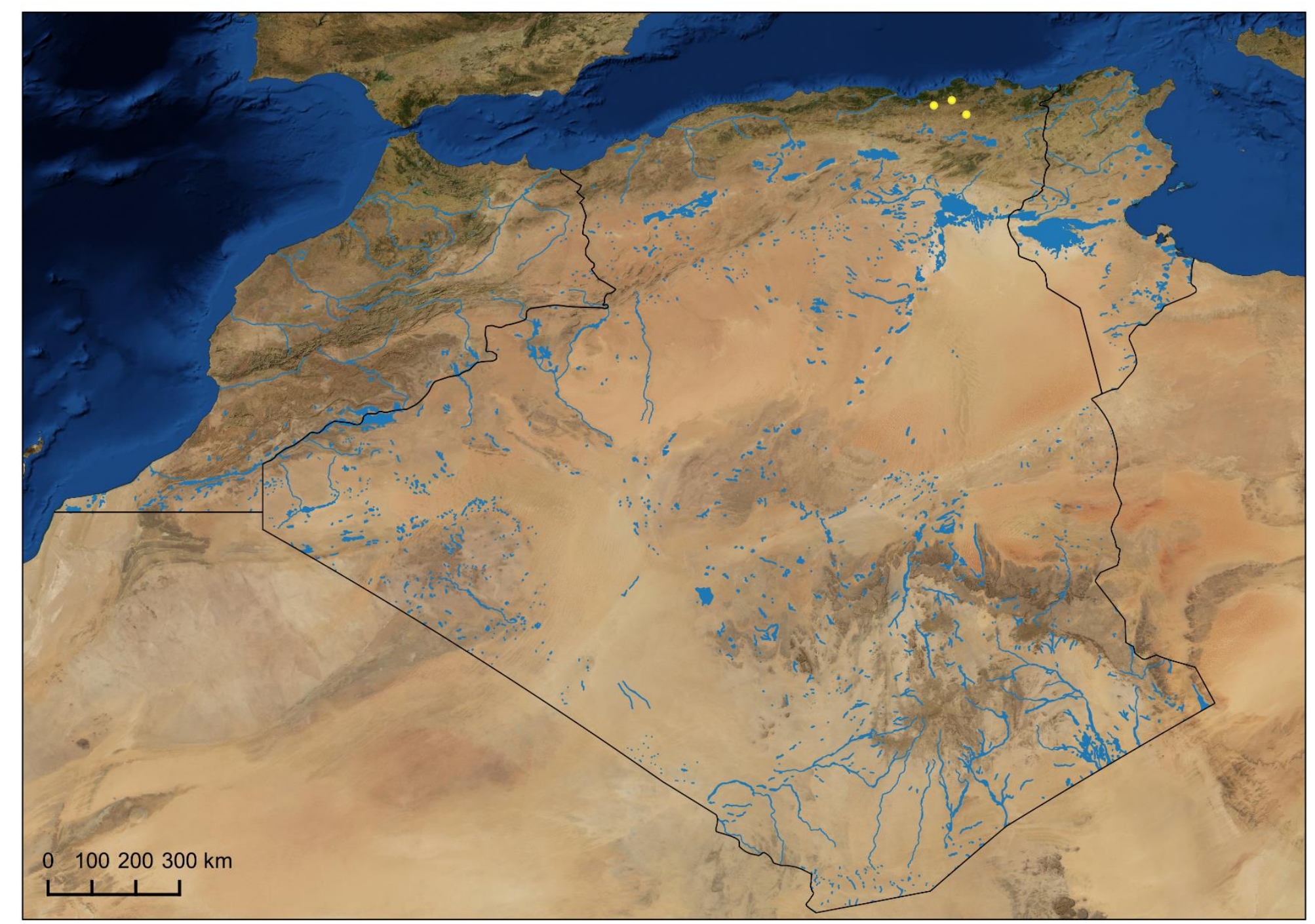

Figure 29: Luciobarbus numidiensis. 


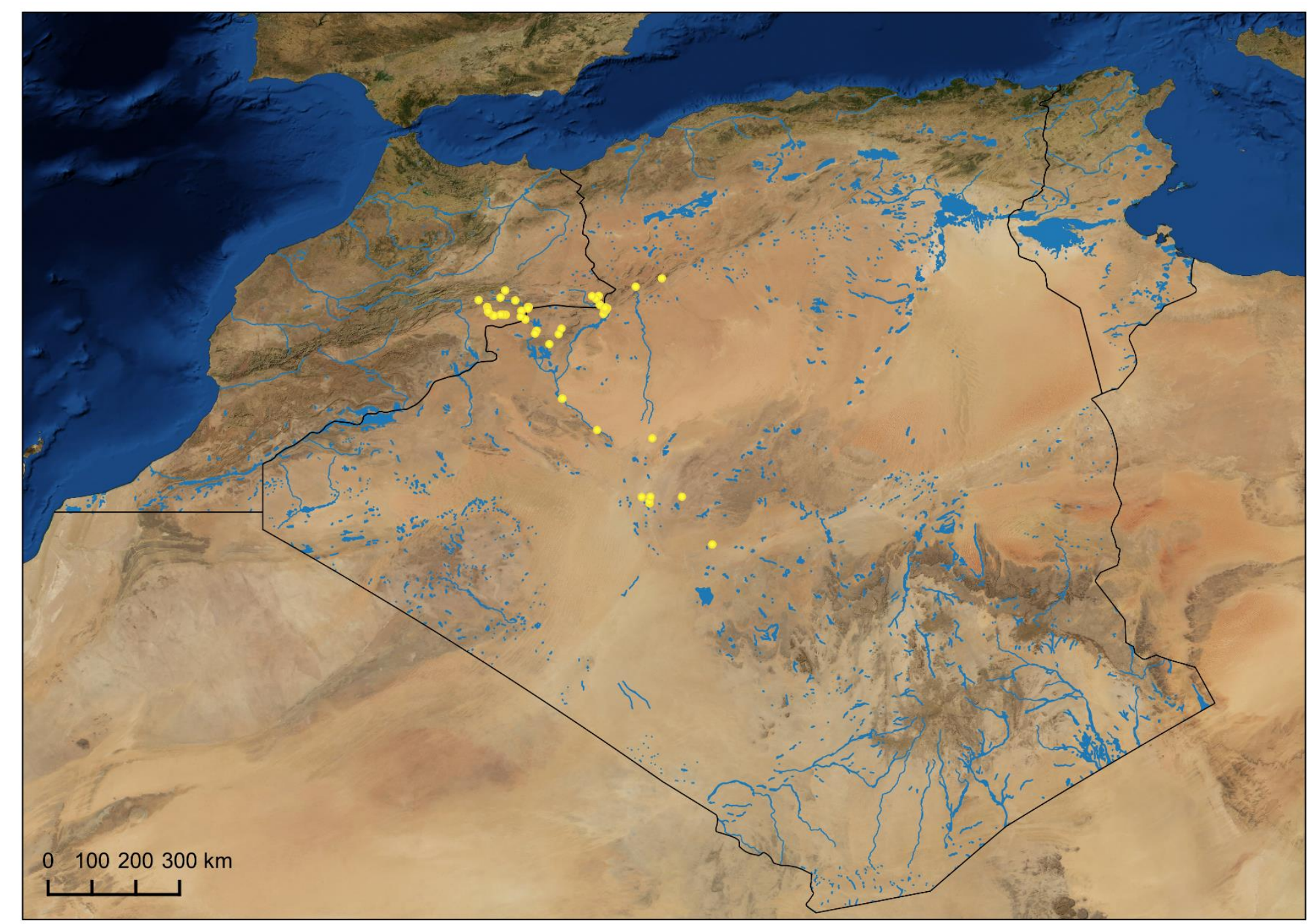

Figure 30: Luciobarbus pallaryi. 


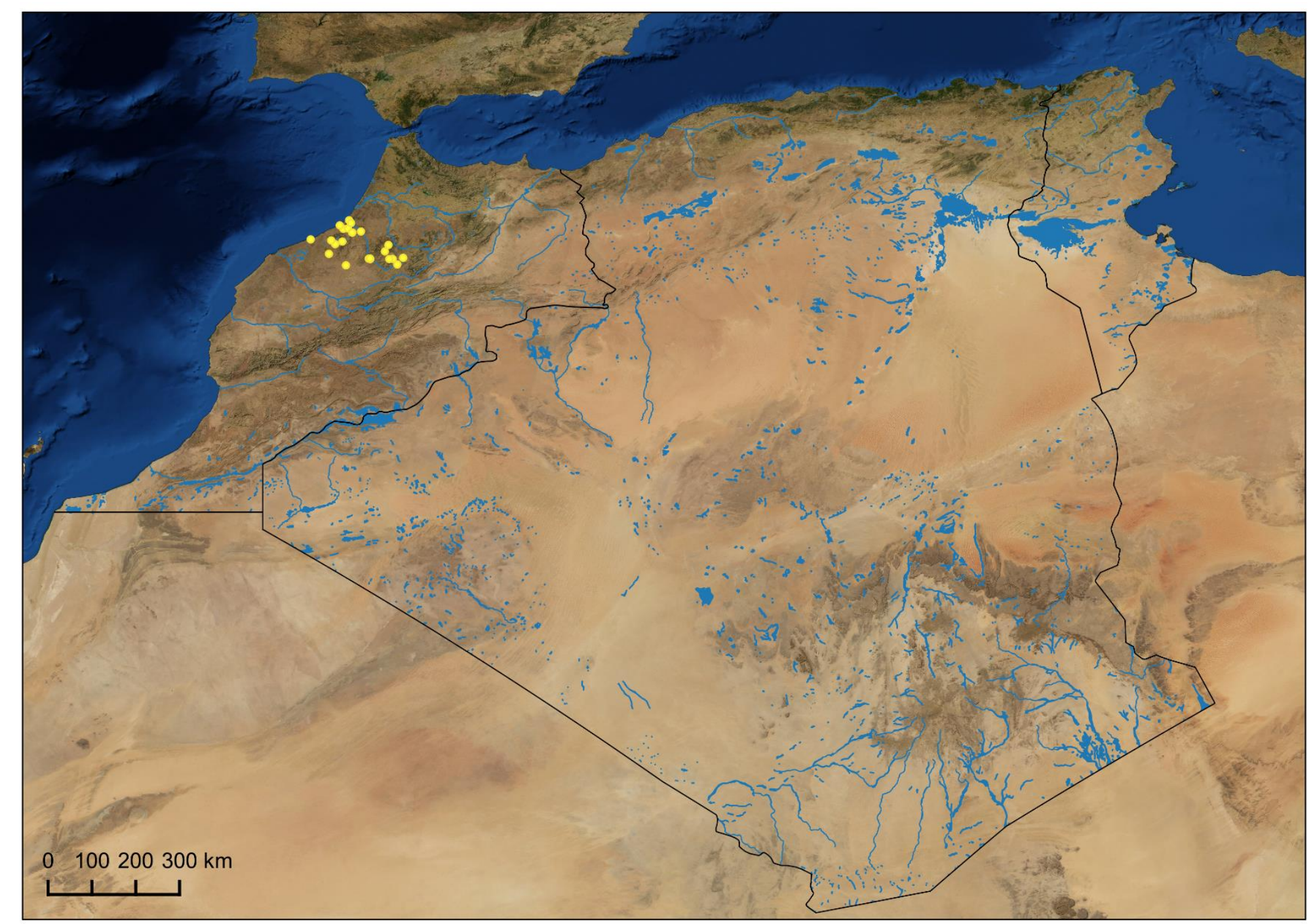

Figure 31: Luciobarbus rabatensis. 


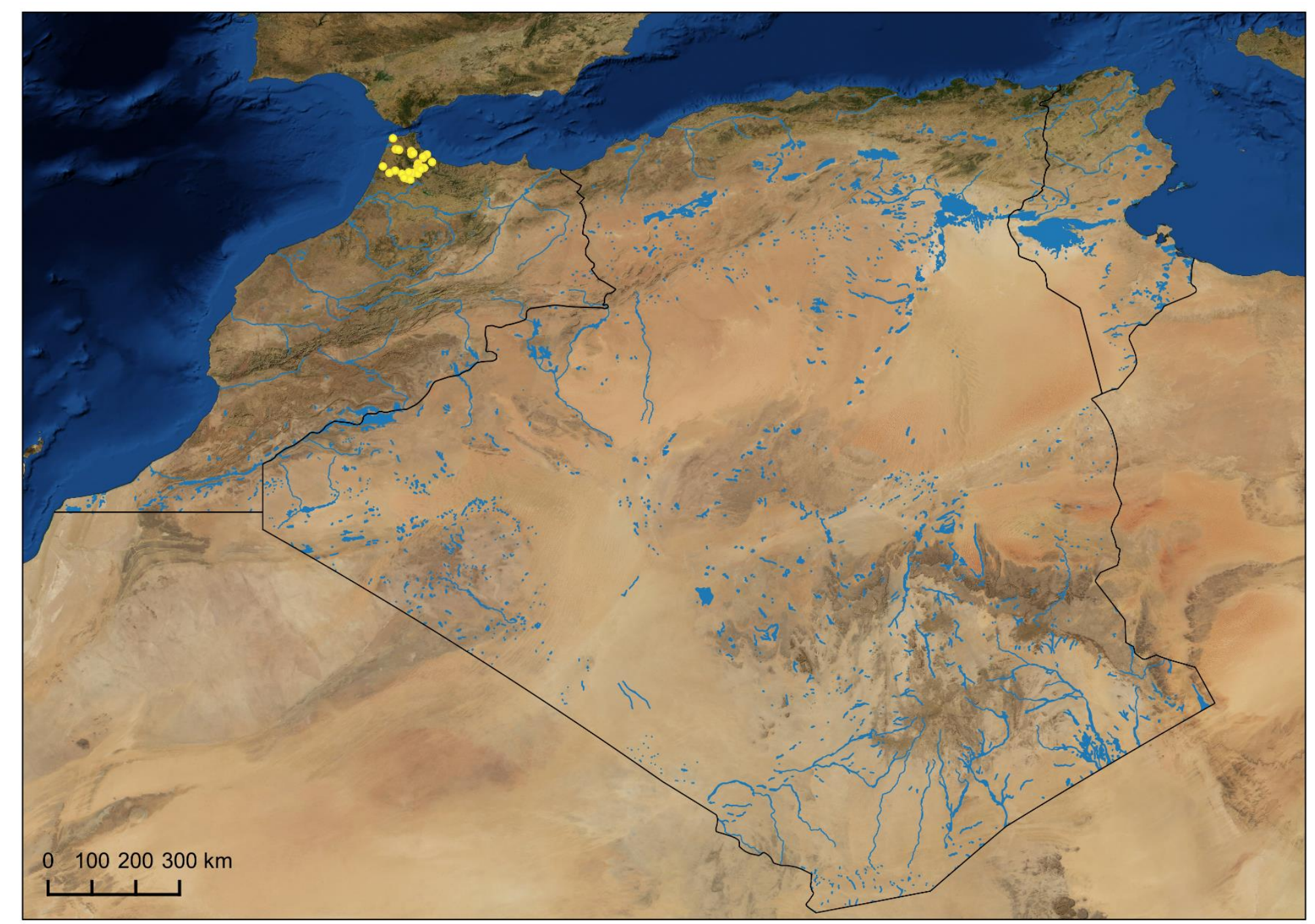

Figure 32: Luciobarbus rifensis. 


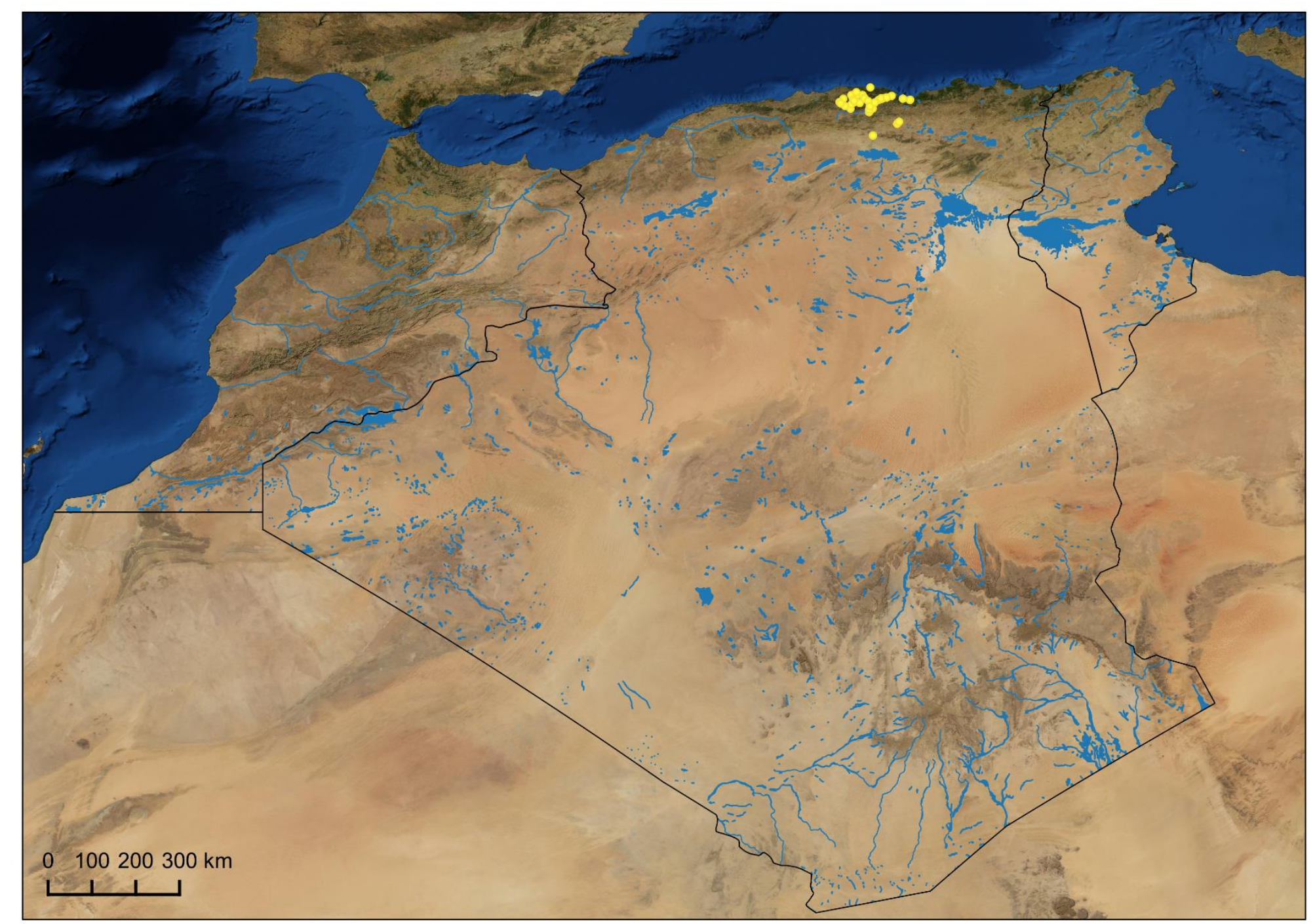

Figure 33: Luciobarbus setivimensis. 


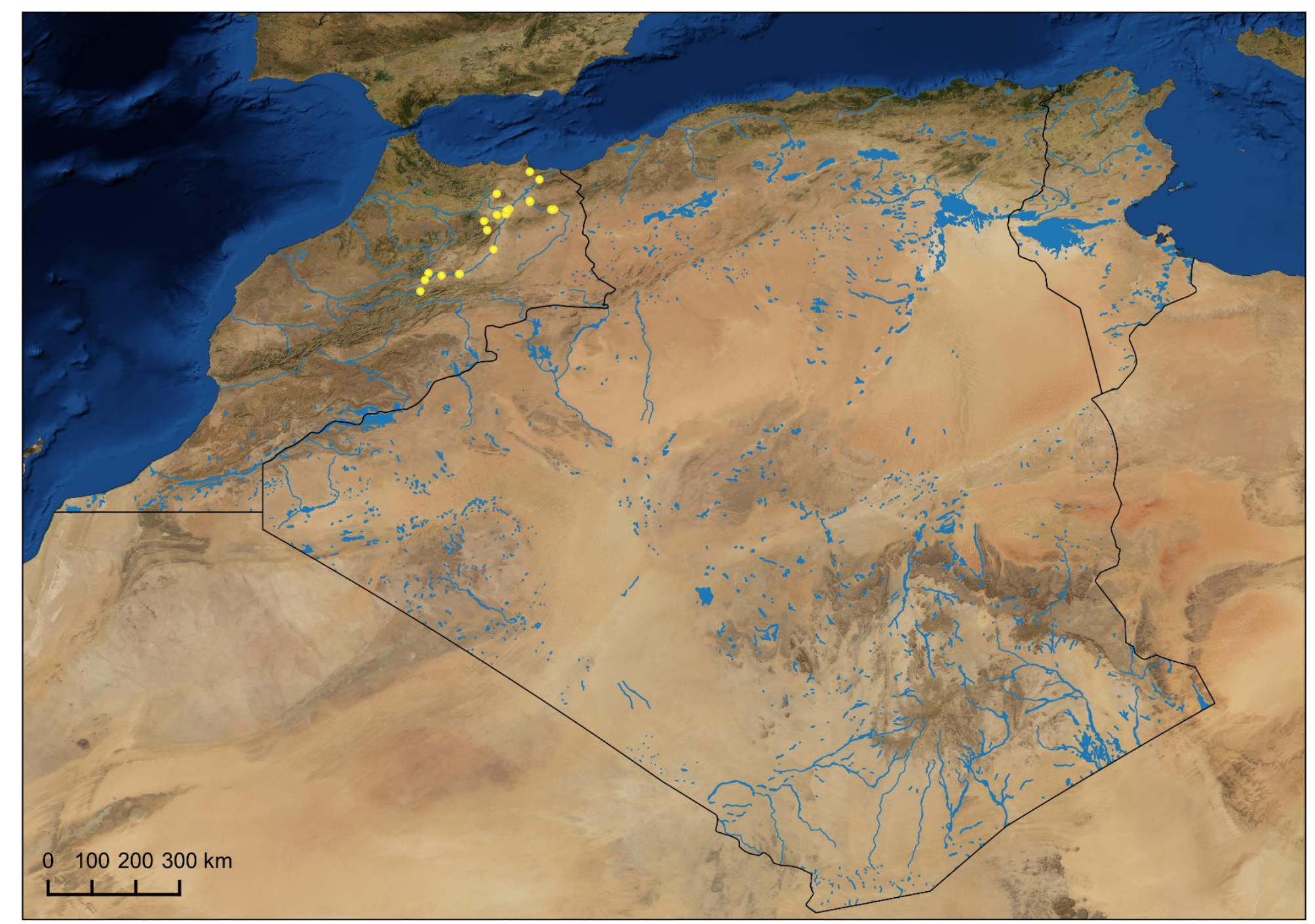

Figure 34: Luciobarbus yahyaouii. 


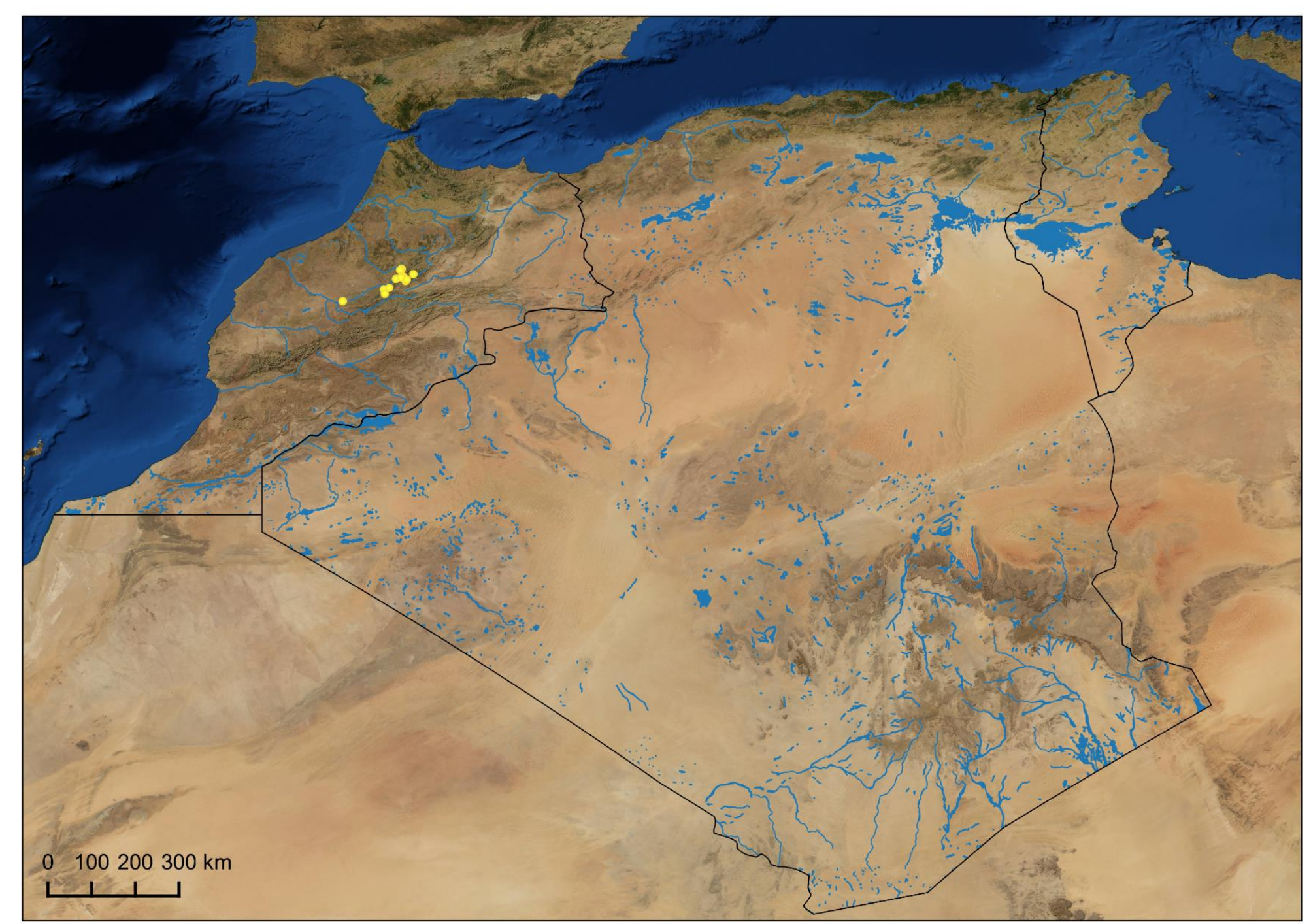

Figure 35: Luciobarbus zayanensis. 


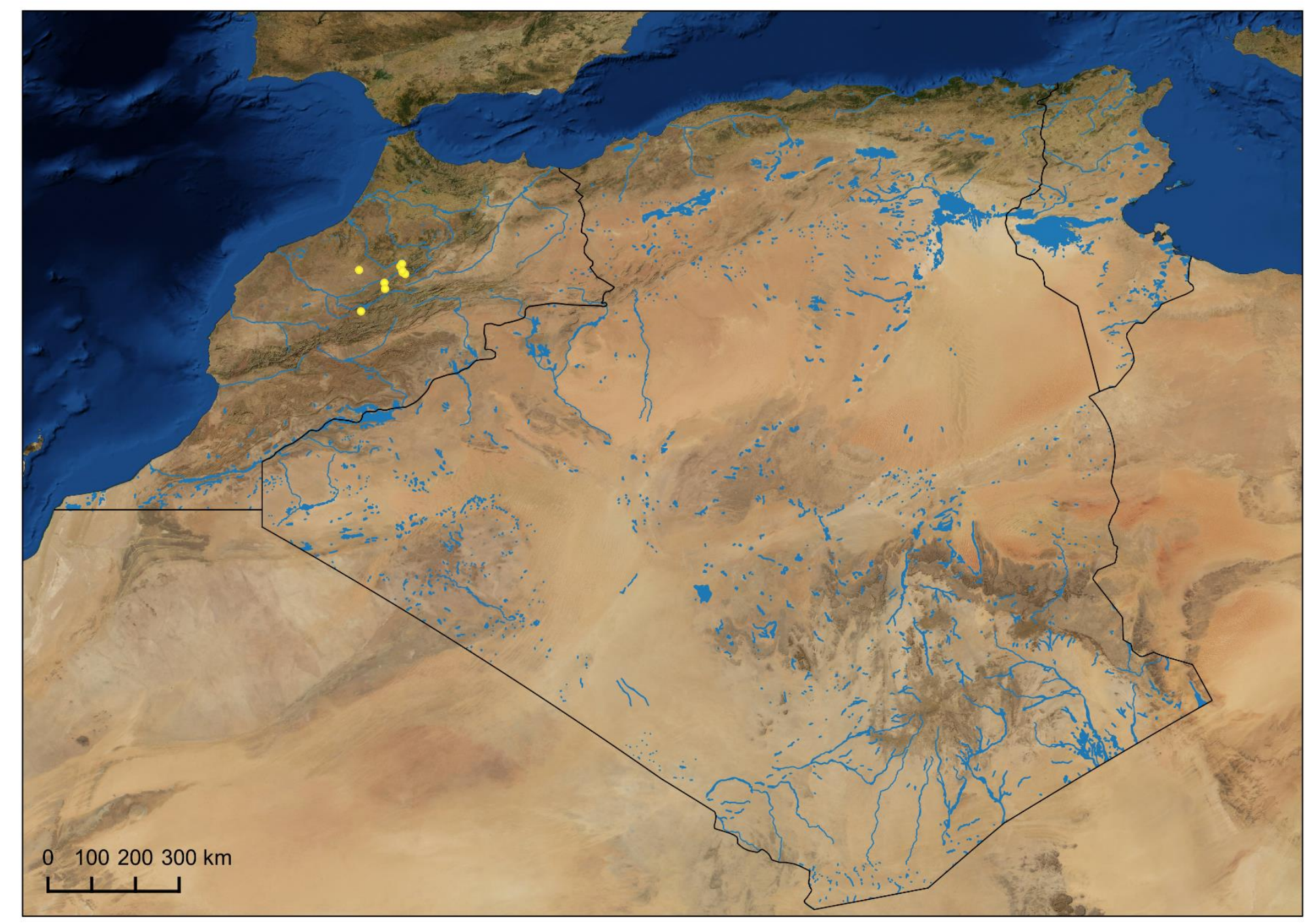

Figure 36: Pterocapoeta maroccana. 


\section{Family: Xenocyprididae}

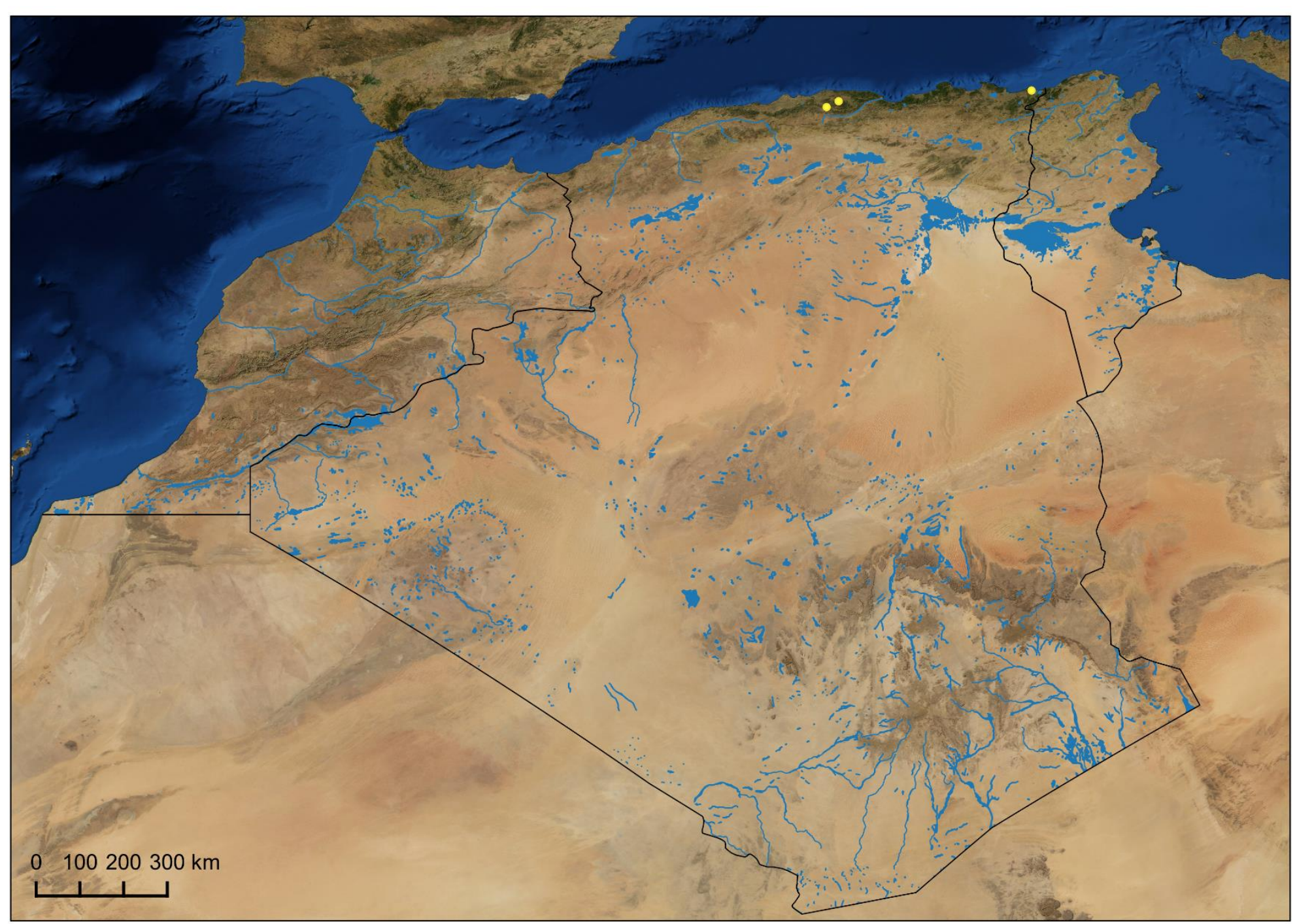

Figure 37: Ctenopharyngodon idella. Introduced throughout much of the region but few precise site-scale records exist. 


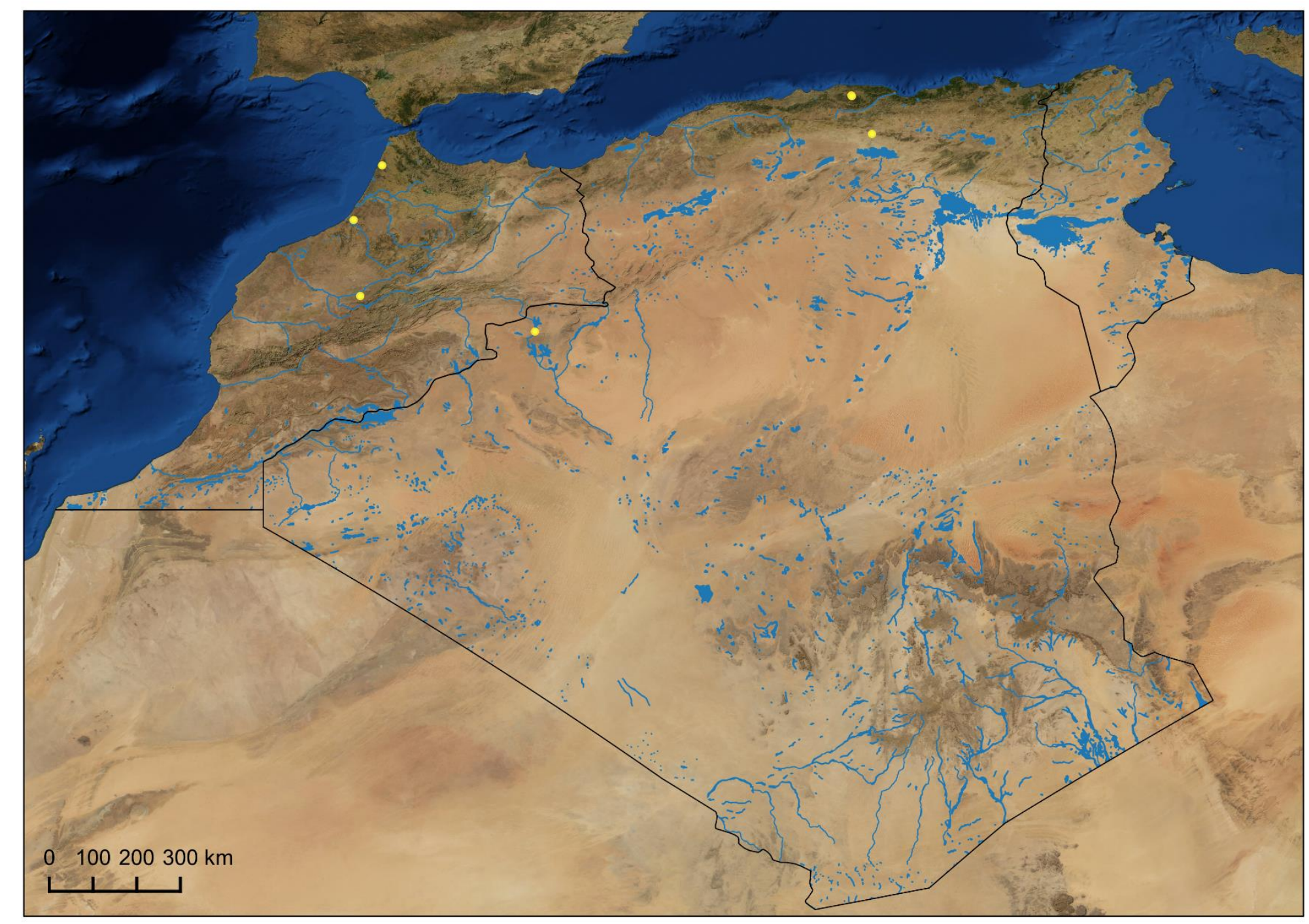

Figure 38: Hypophthalmichthys molitrix. Introduced throughout much of the region but few precise site-scale records exist. 


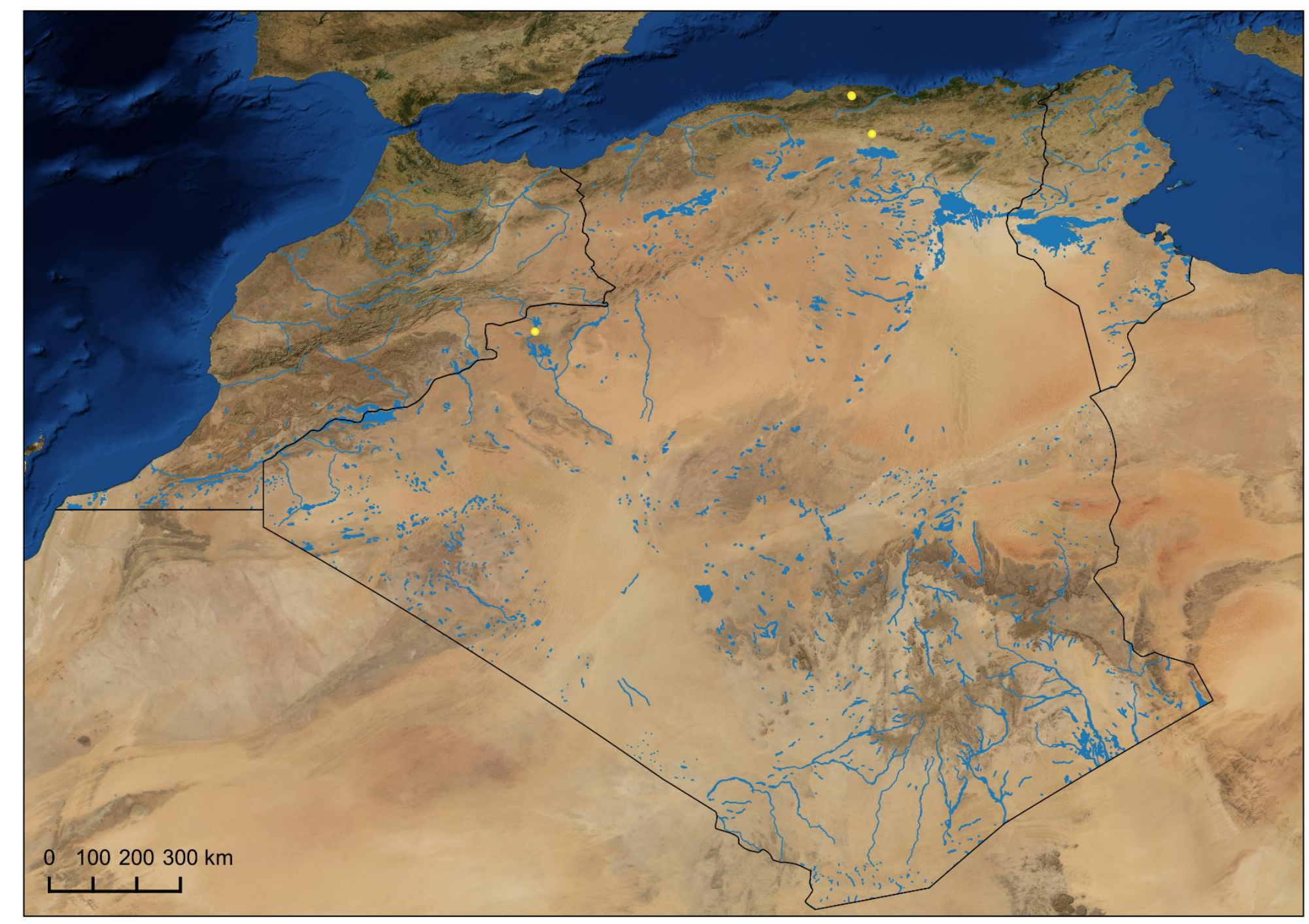

Figure 39: Hypophthalmichthys nobilis. Introduced throughout much of the region but few precise site-scale records exist. 


\section{Family: Leuciscidae}

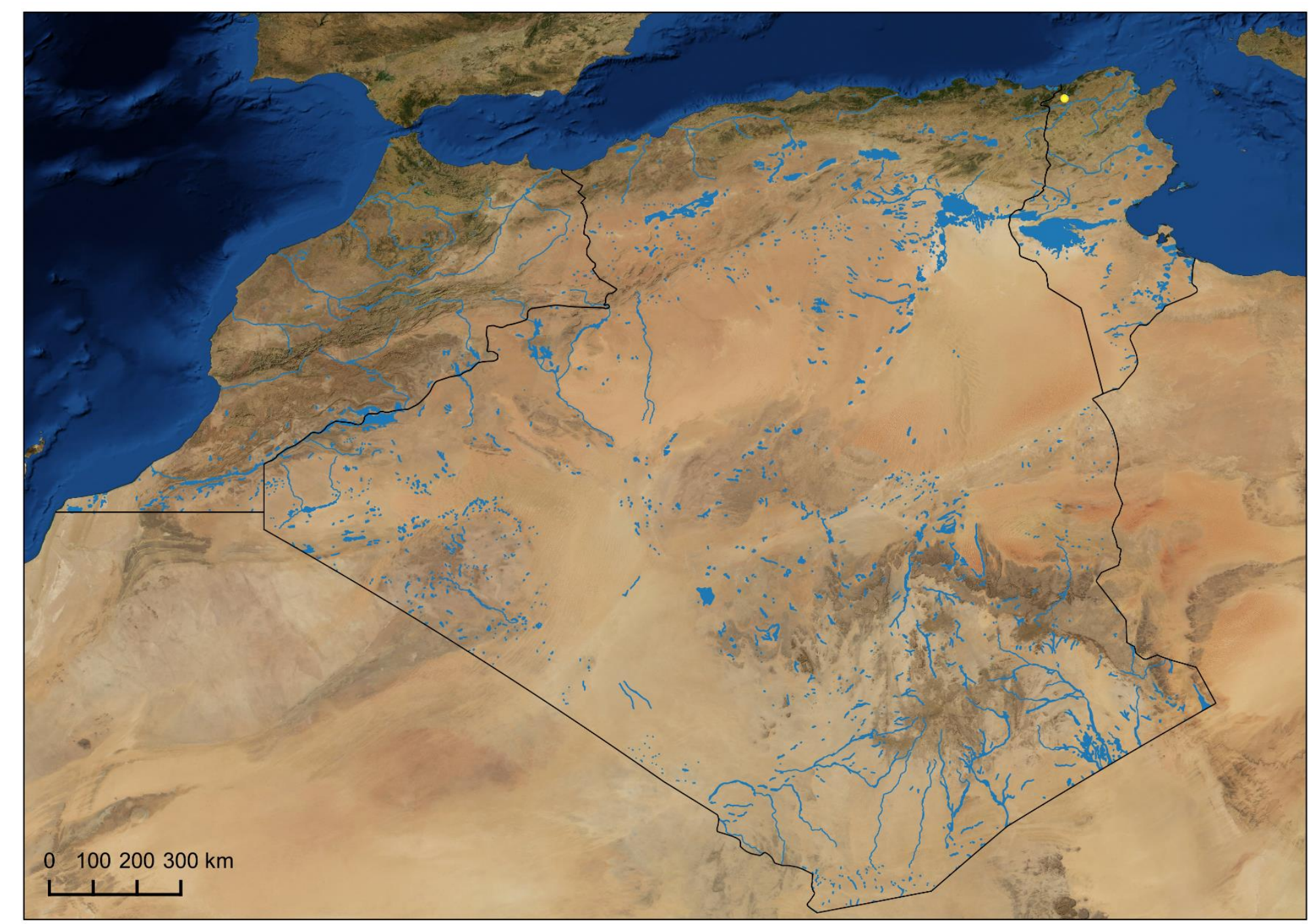

Figure 40: Anaecypris punica. Expected to be more widespread in Tunisia and northeastern Algeria. 


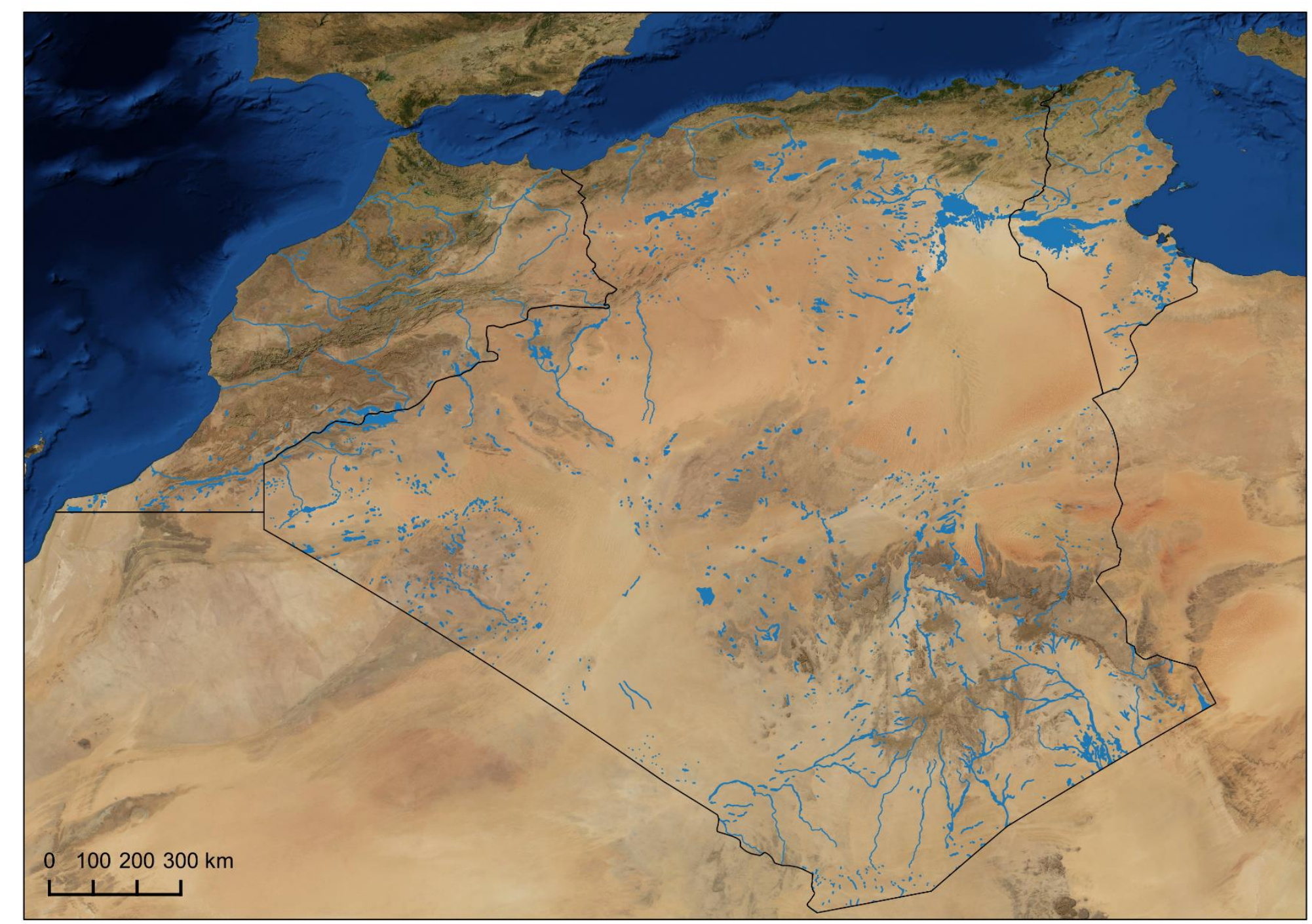

Figure 41: Rutilus rubilio. Reportedly introduced to Tunisia, but no precise site-scale records are available. 


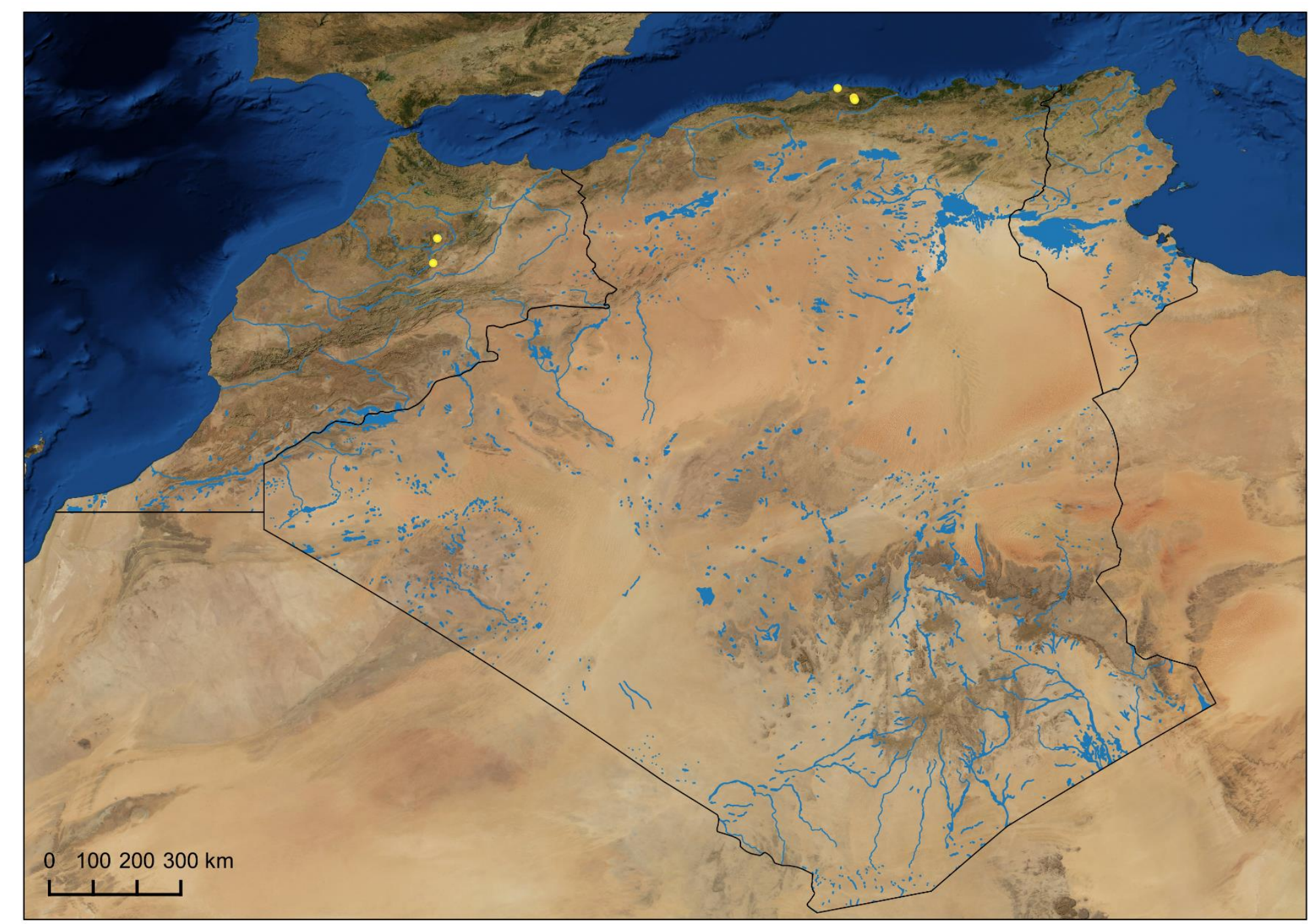

Figure 42: Rutilus rutilus. Recently recorded in the region for the first time and may occur elsewhere. 


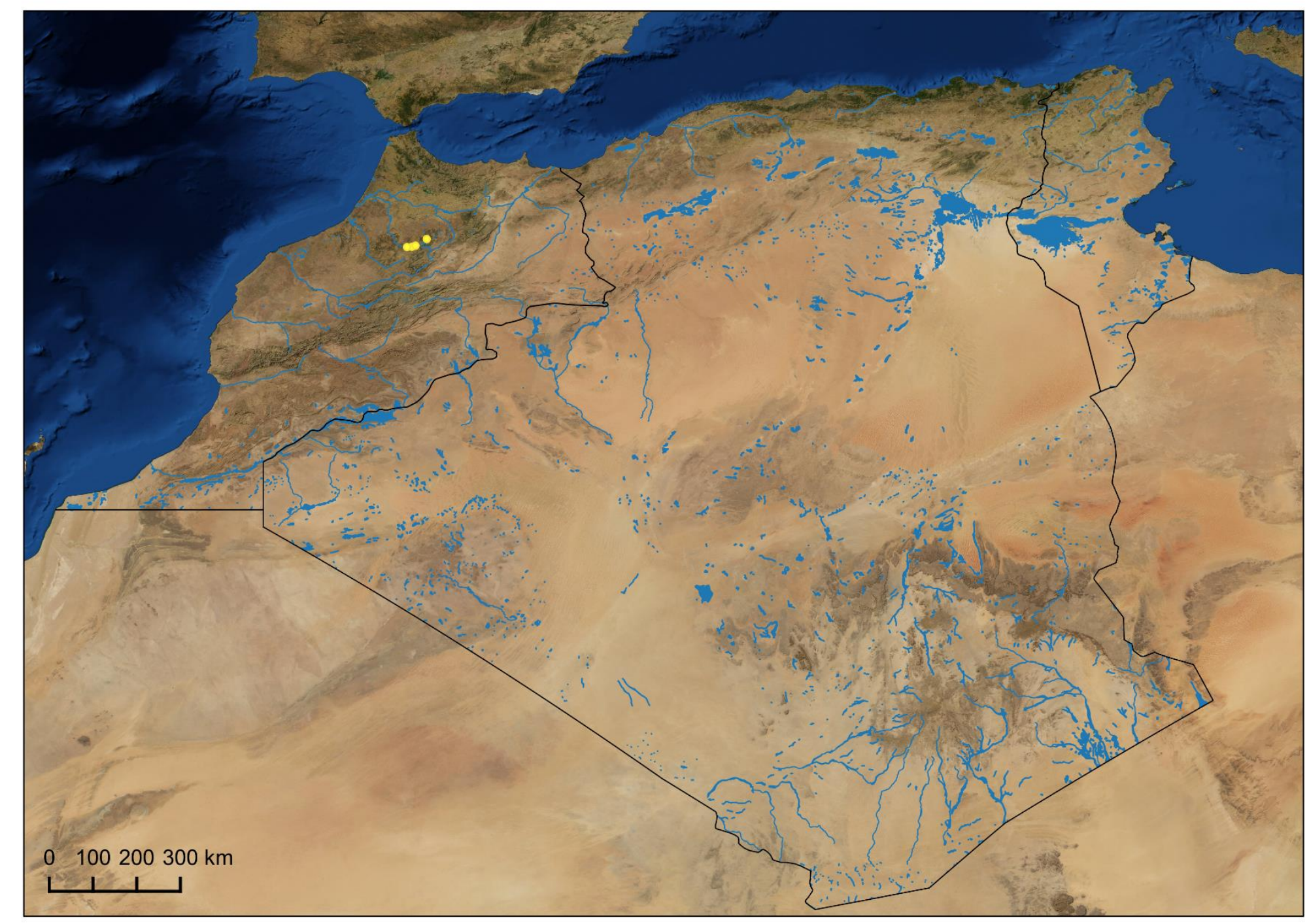

Figure 43: Scardinius erythrophthalmus. Recently recorded in the region for the first time and may occur elsewhere. 


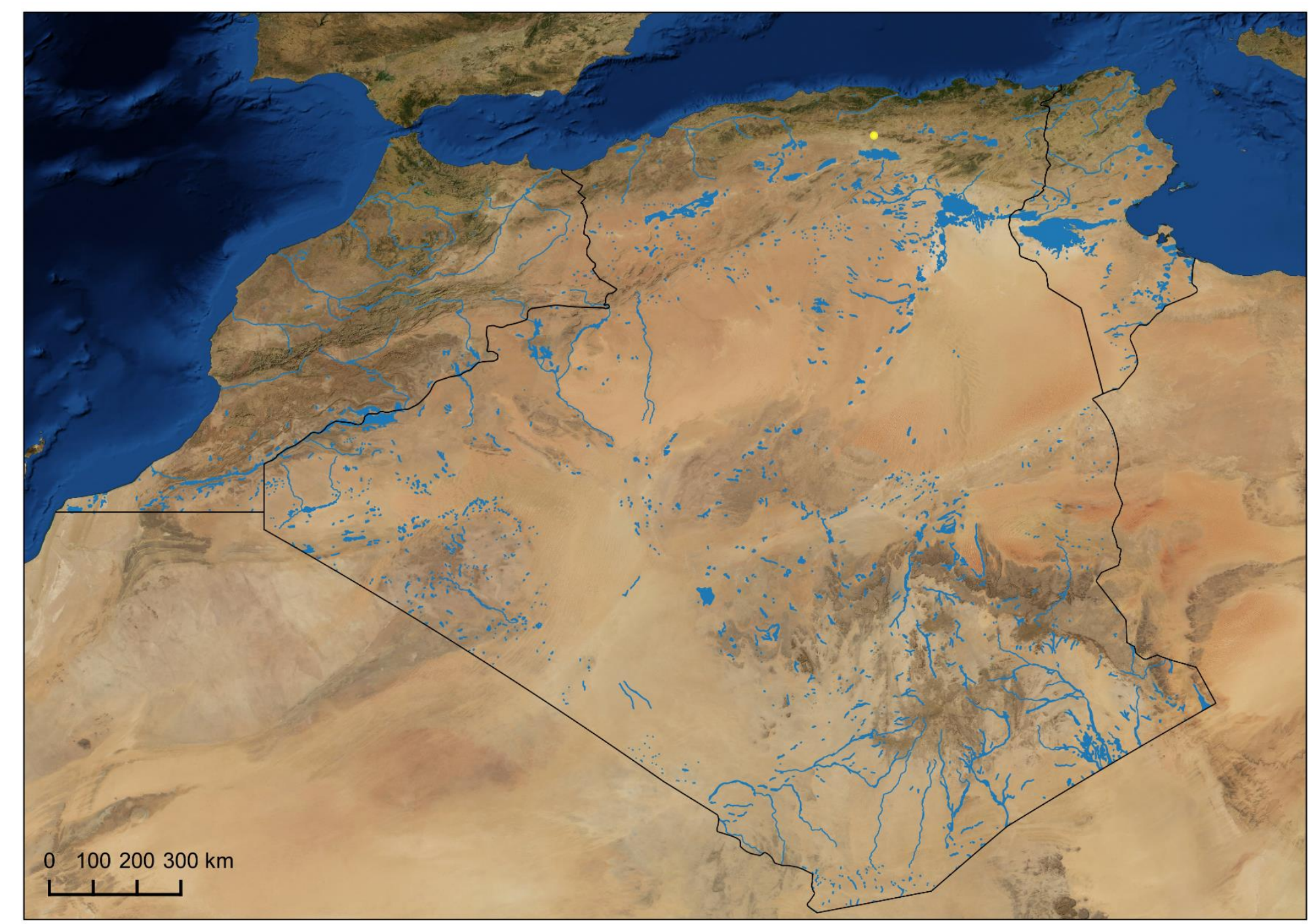

Figure 44: Squalius cephalus. Recently recorded in the region for the first time and may occur elsewhere. 


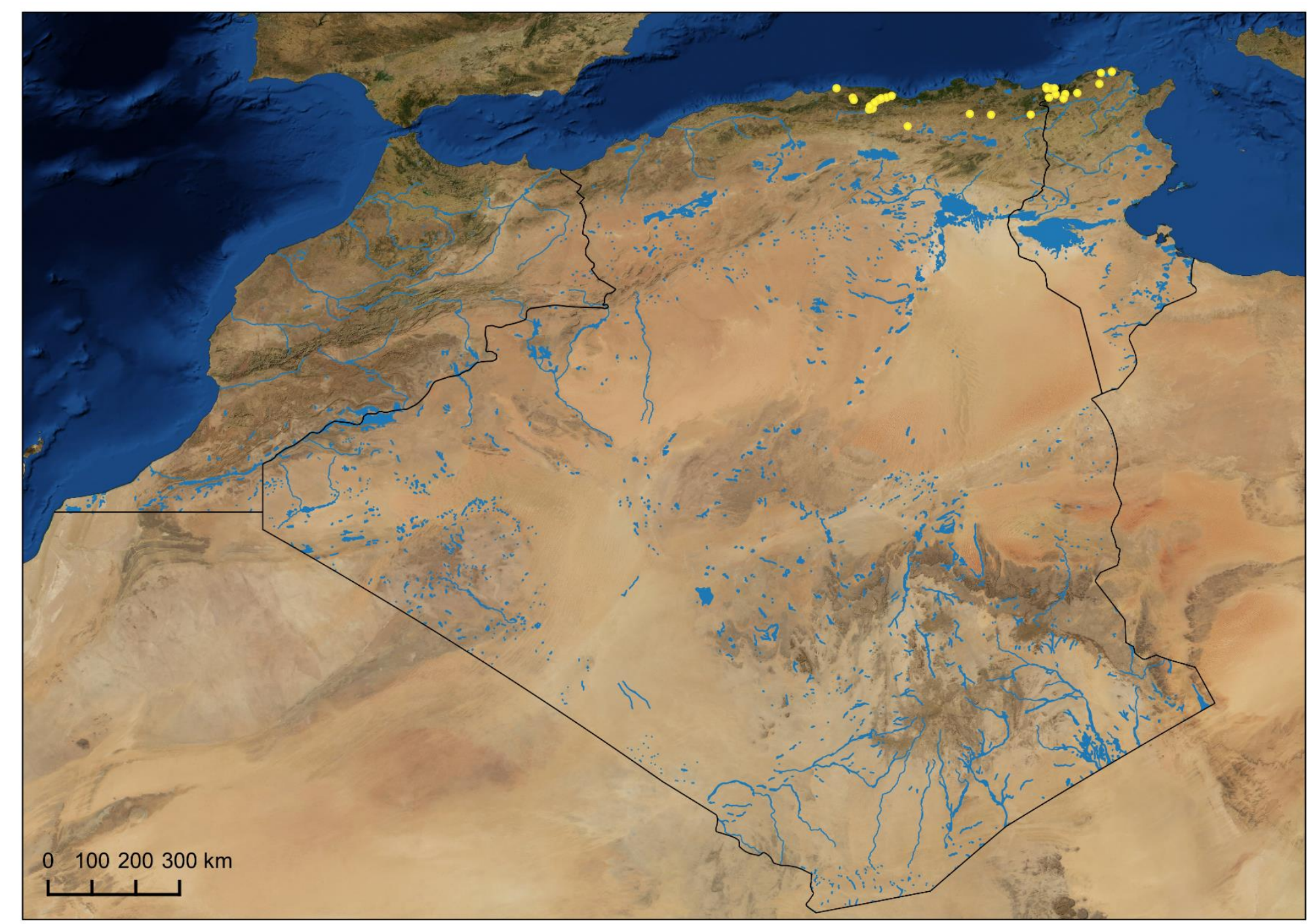

Figure 45: Tropidophoxinellus callensis. 


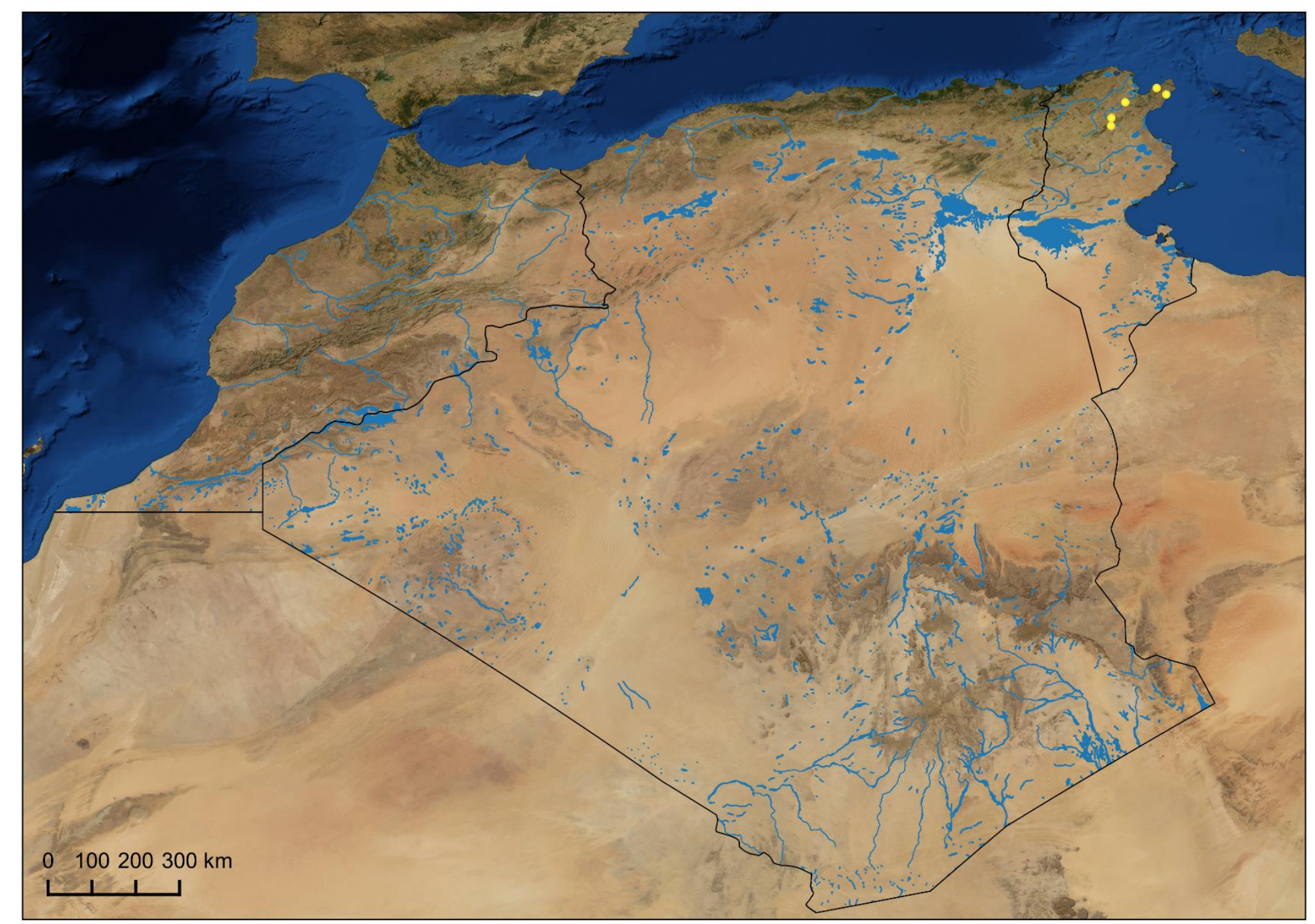

Figure 46: Tropidophoxinellus chaignoni. 


\section{Family: Tincidae}

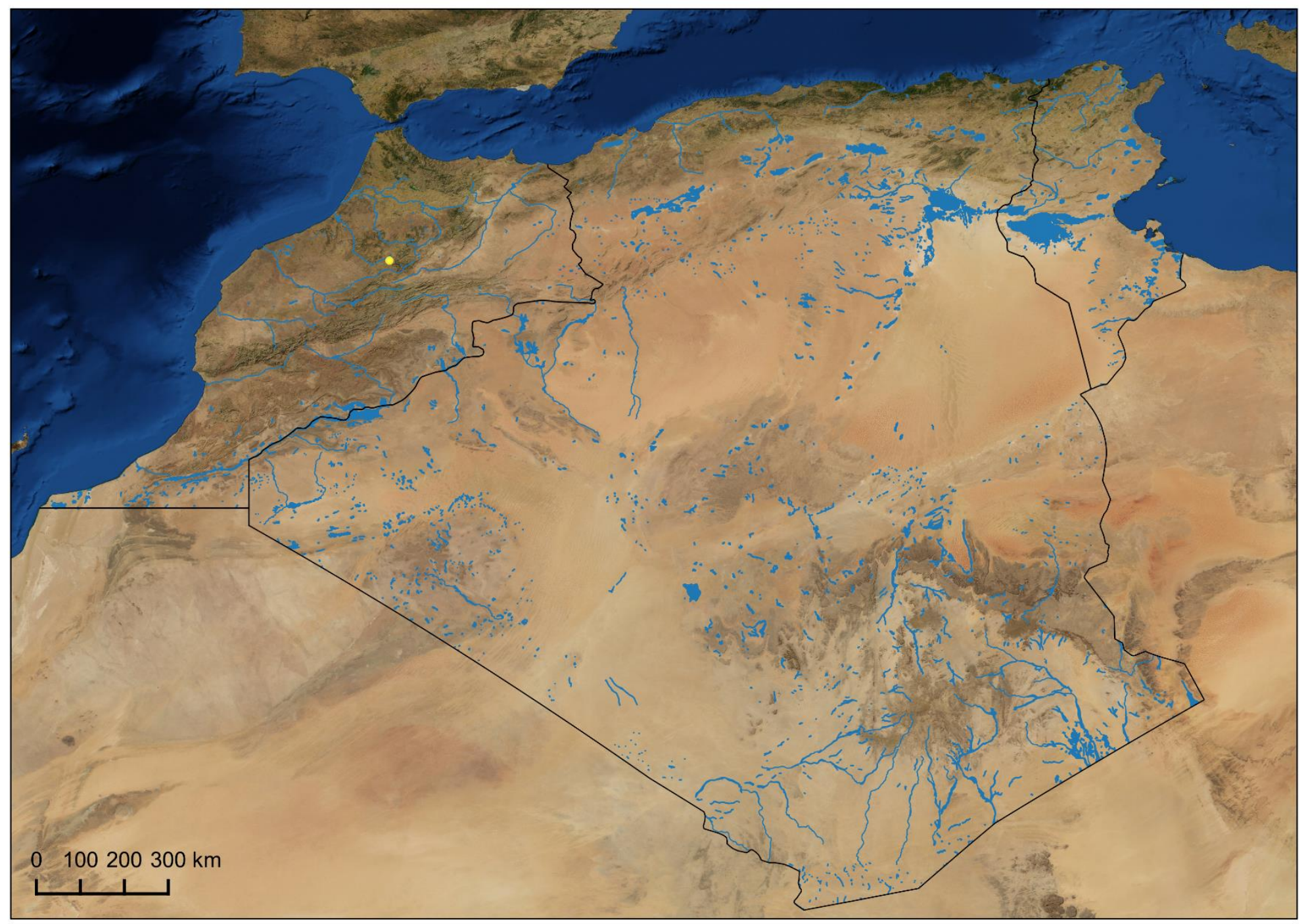

Figure 47: Tinca tinca. Introduced throughout a substantial portion of the region but few precise site-scale records exist. 


\section{Family: Cobitidae}

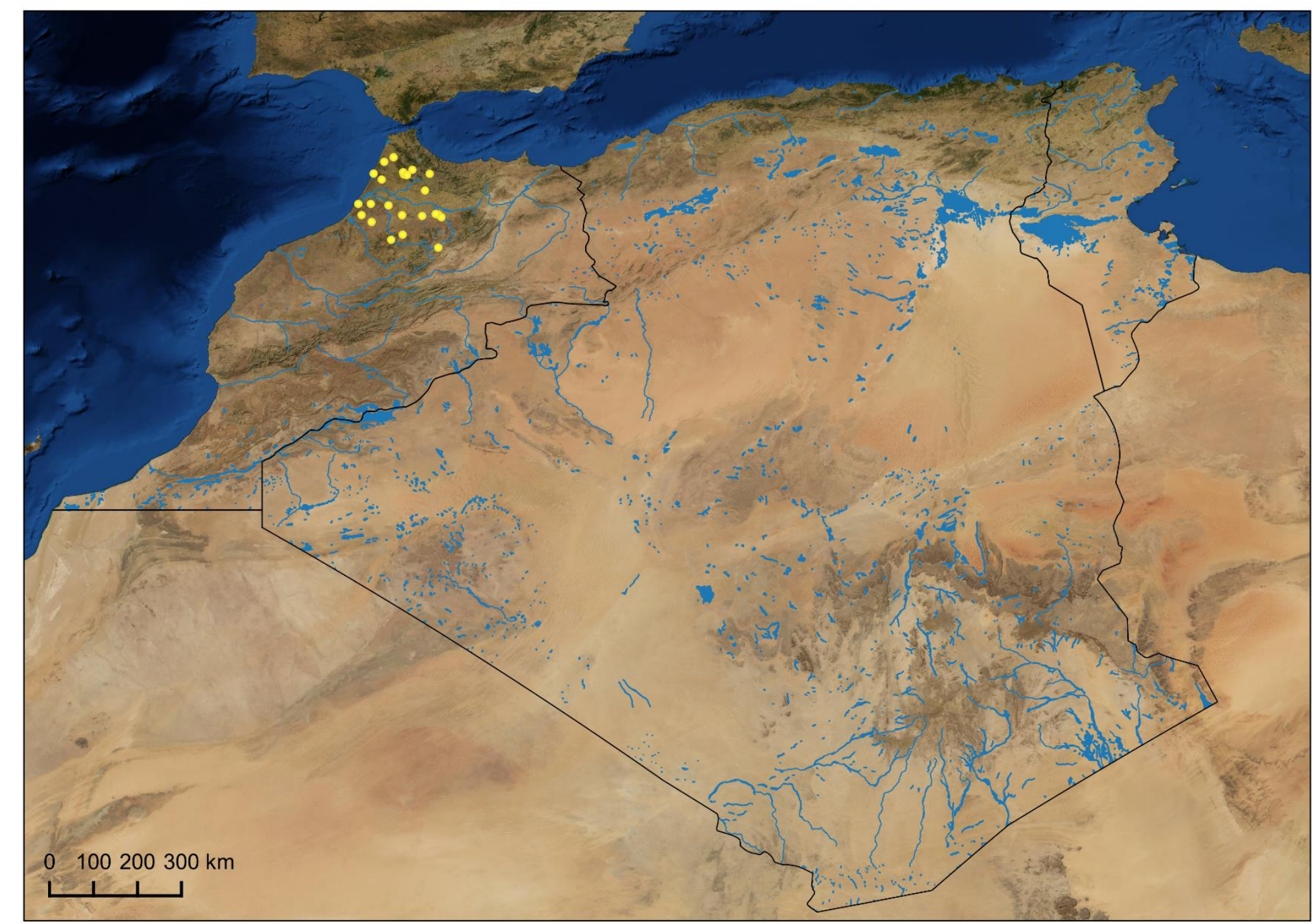

Figure 48: Cobitis maroccana. 


\section{Family: Gobionidae}

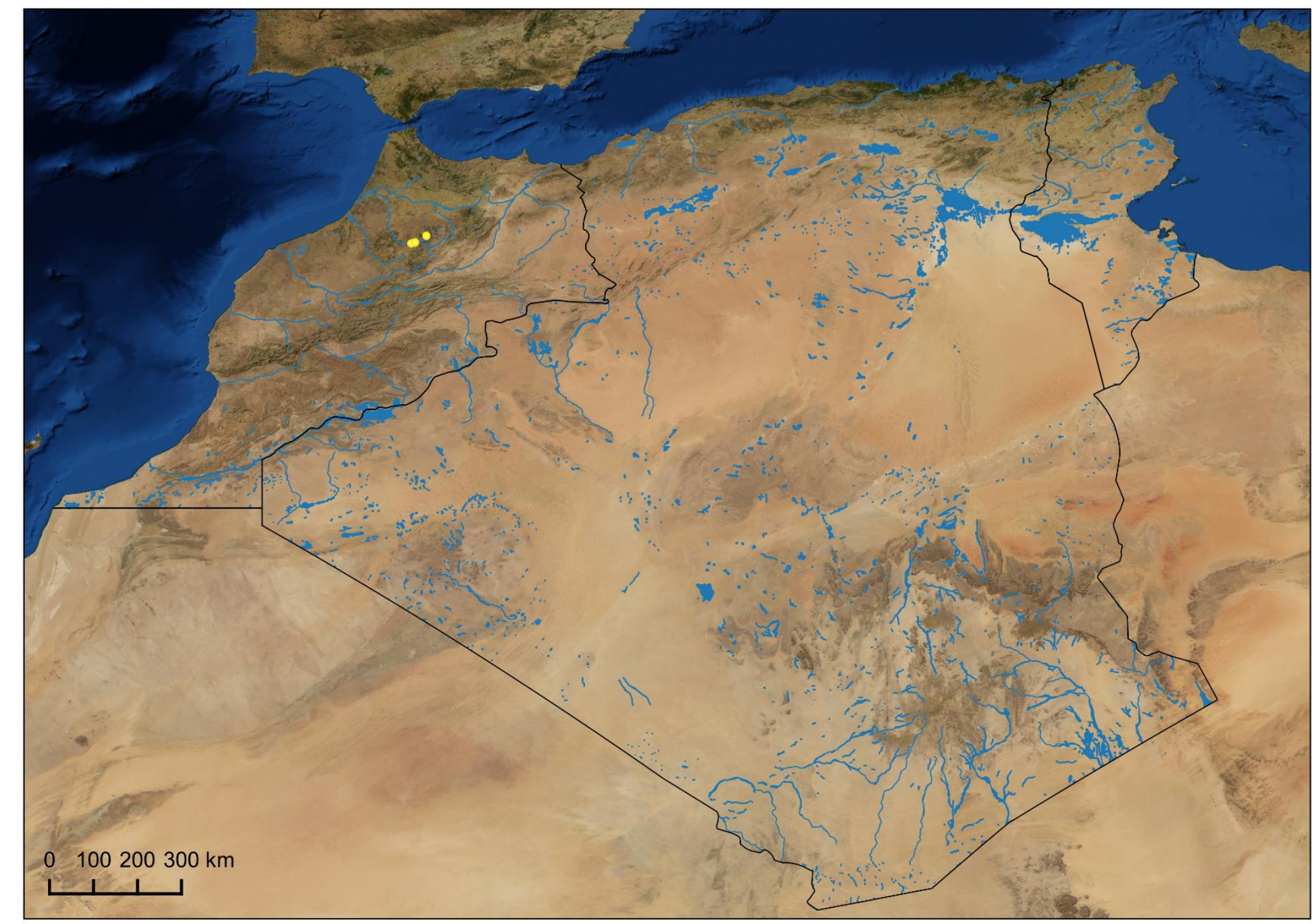

Figure 49: Gobio gobio. Recently recorded in the region for the first time and may occur elsewhere. 


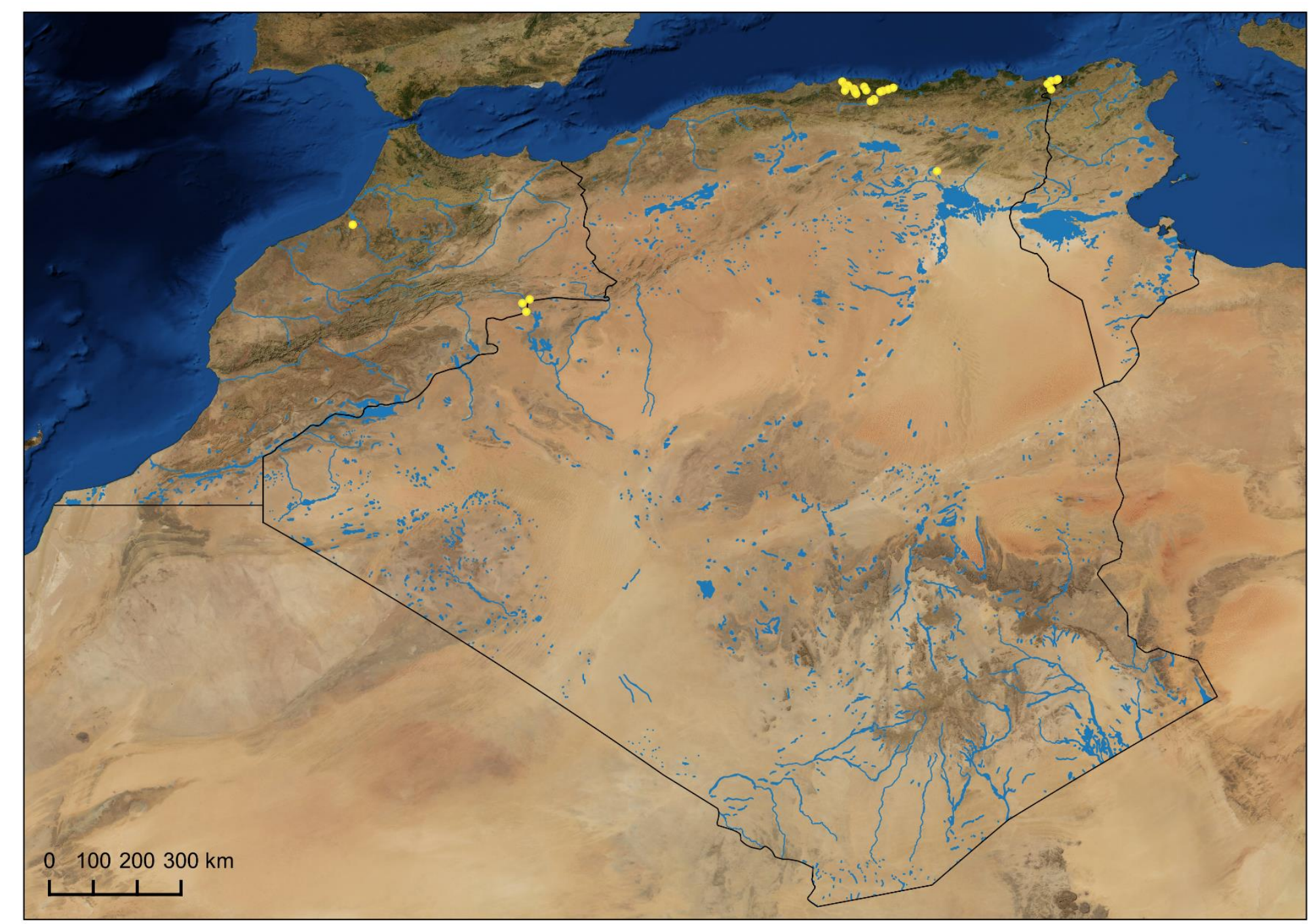

Figure 50: Pseudorasbora parva. Introduced throughout a substantial portion of the region but few site-scale records exist. 


\section{Family: Clariidae}

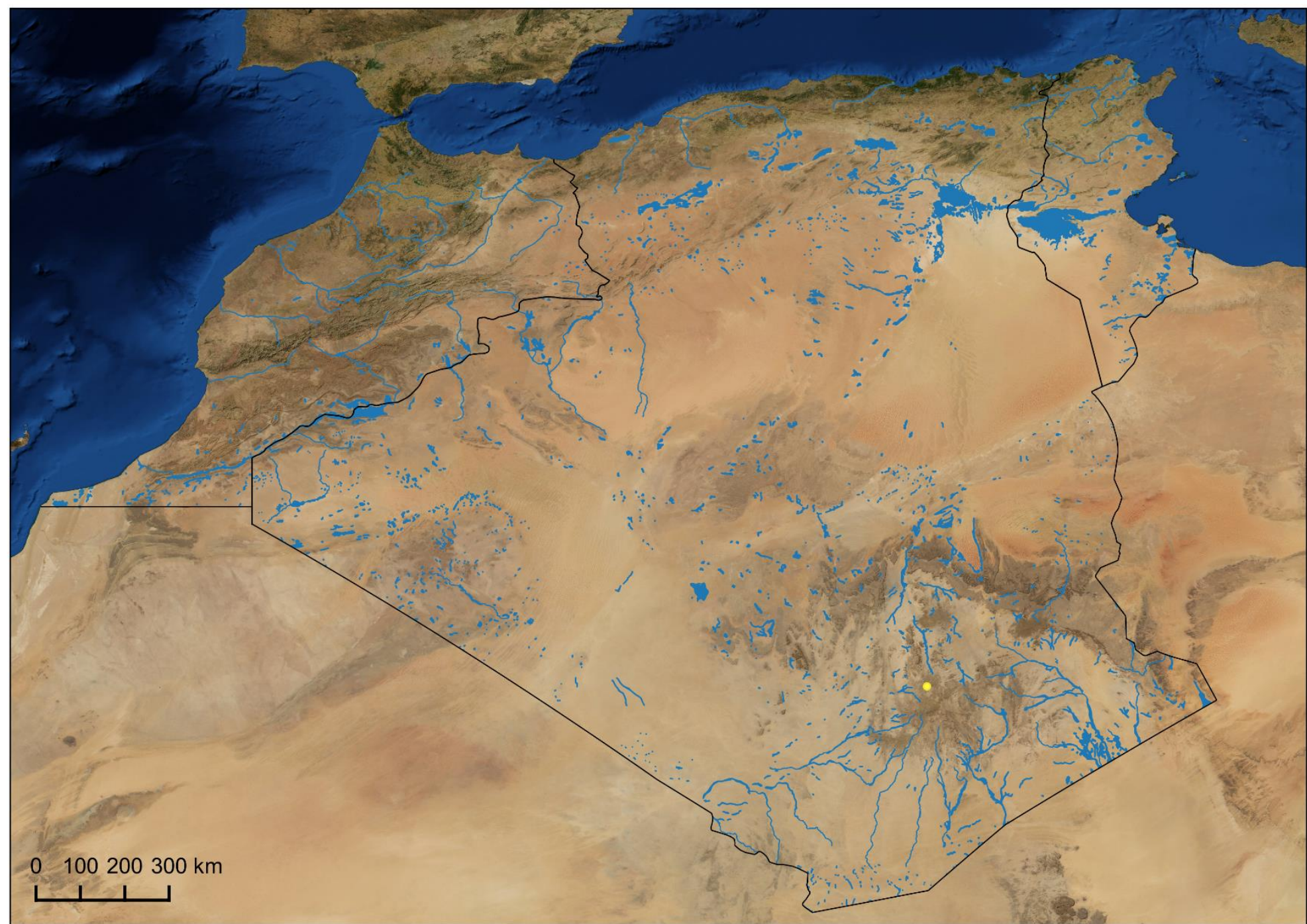

Figure 51: Clarias anguillaris. Also occurs along the northern margin of the Sahara Desert. 


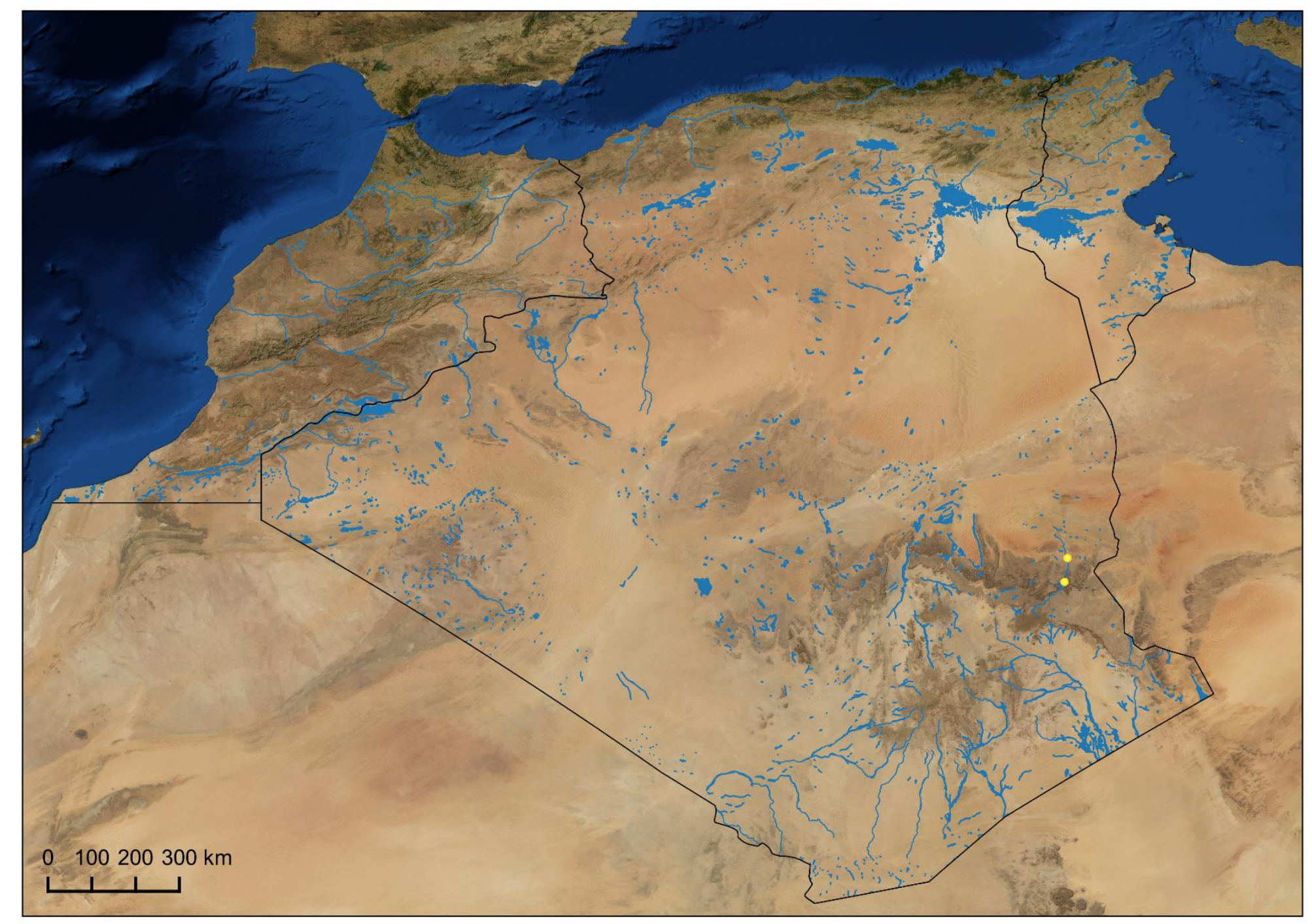

Figure 52: Clarias gariepinus. Also occurs along the northern margin of the Sahara Desert. 


\section{Family: Esocidae}

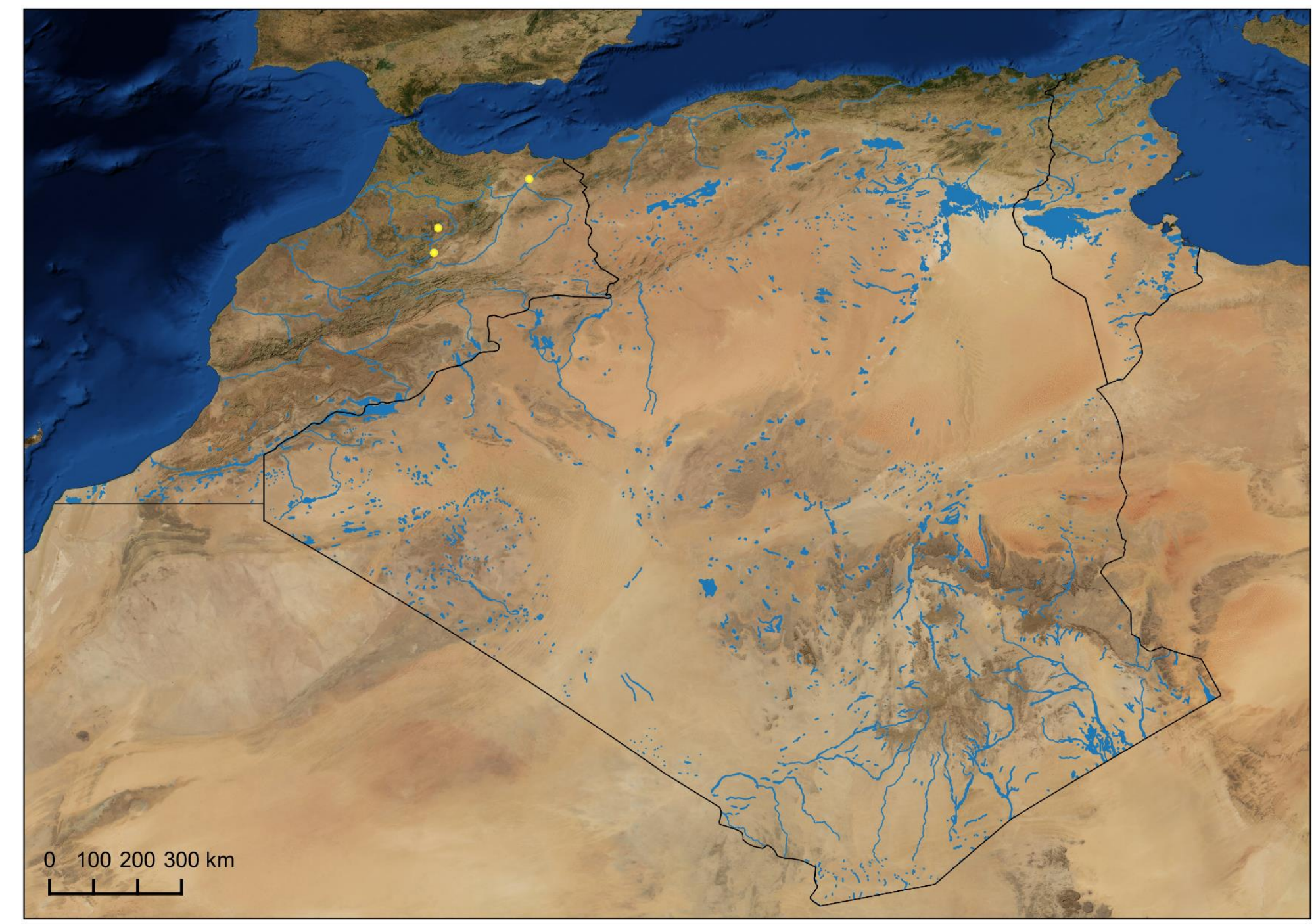

Figure 53: Esox lucius. Recently recorded in the region for the first time and may occur elsewhere. 


\section{Family: Salmonidae}

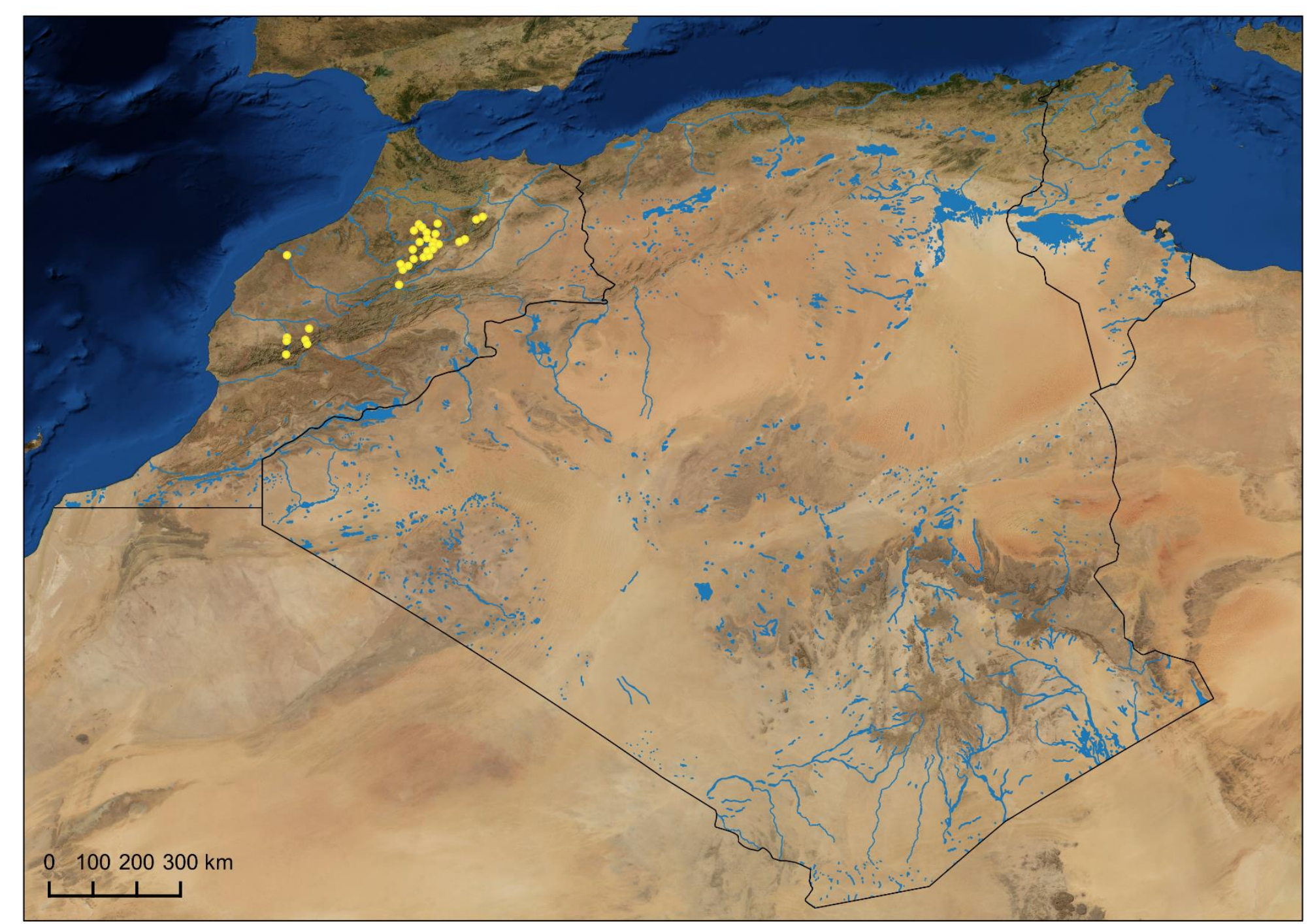

Figure 54: Oncorhynchus mykiss. 


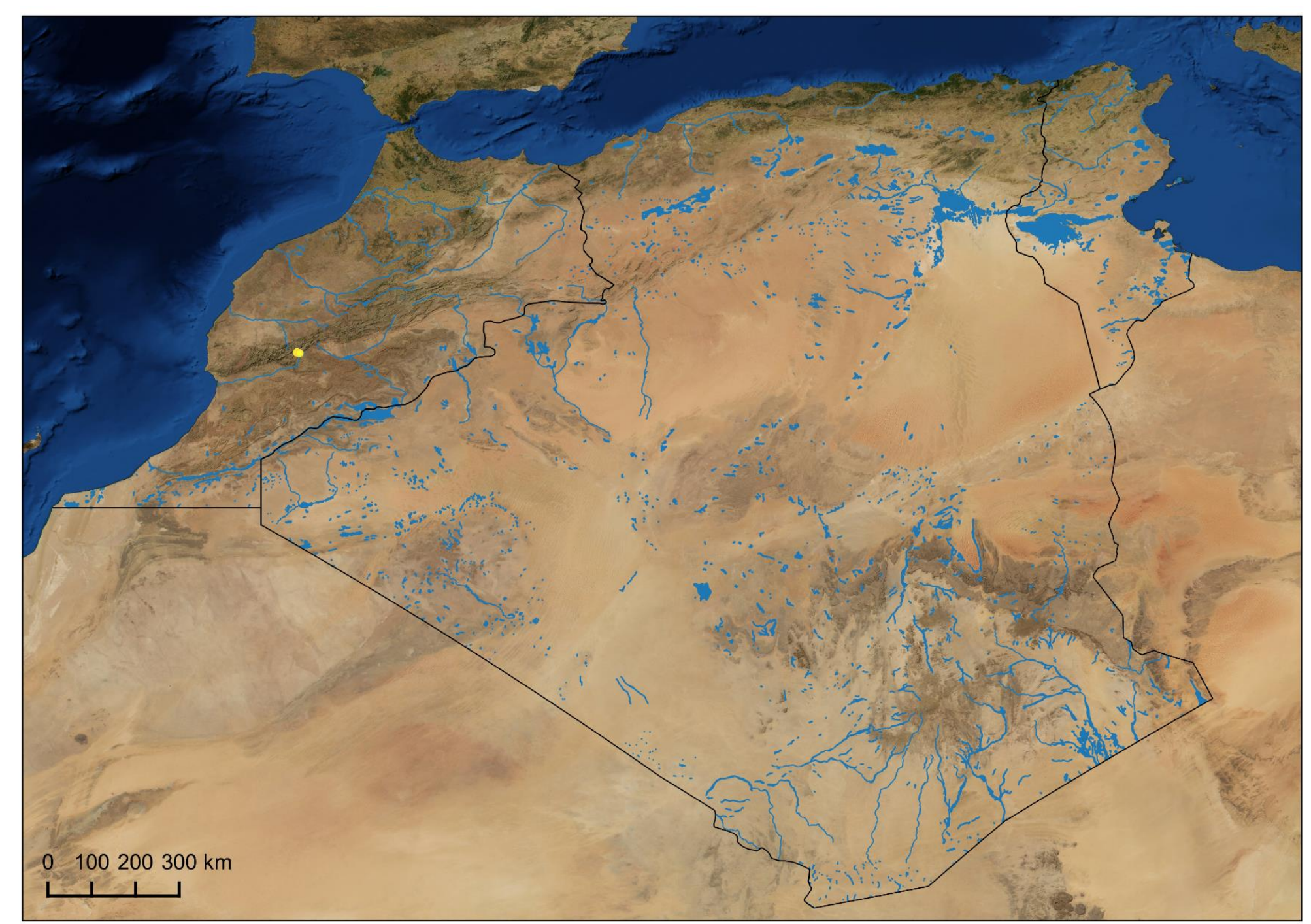

Figure 55: Salmo akairos. 


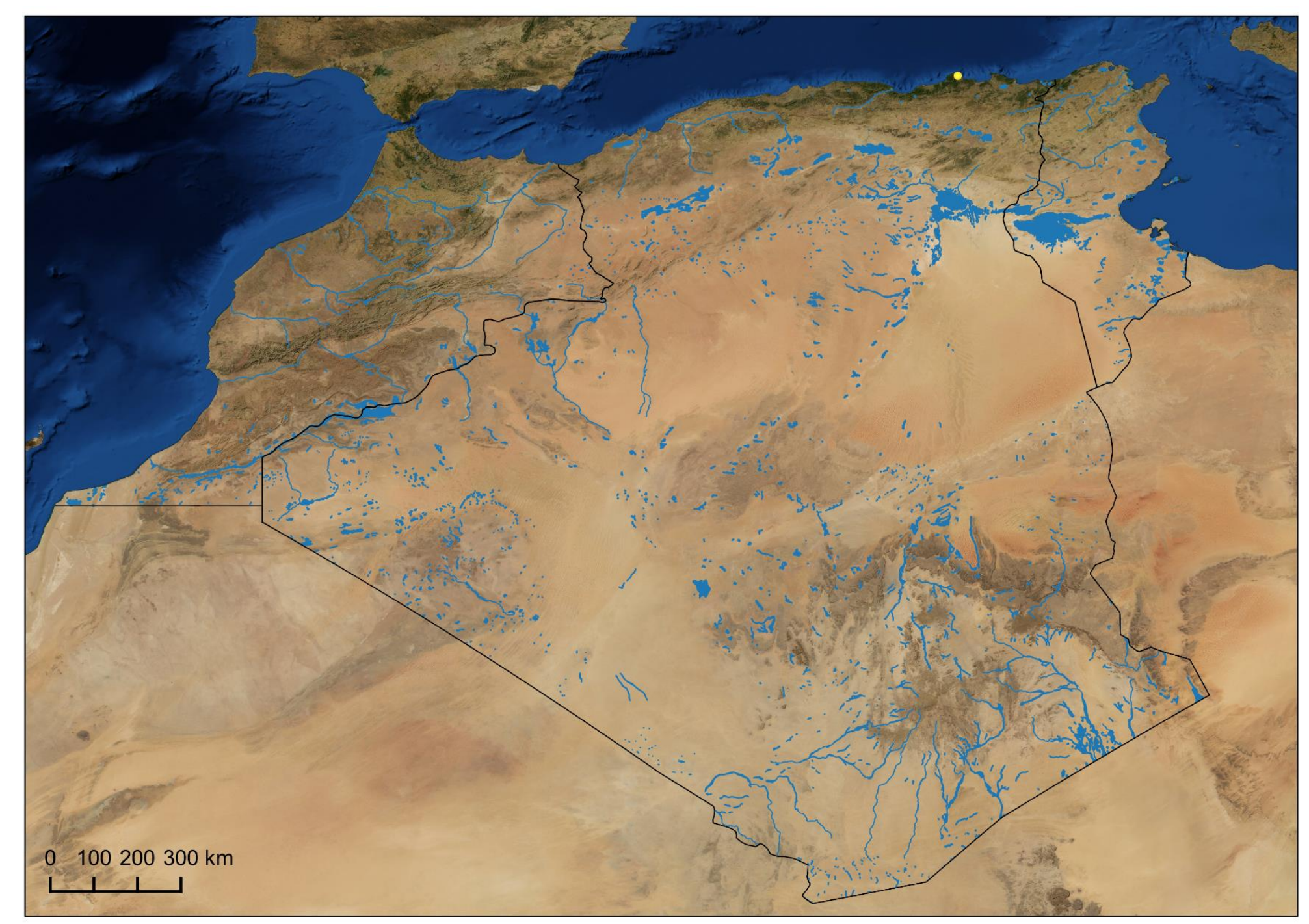

Figure 56: Salmo cettii. 


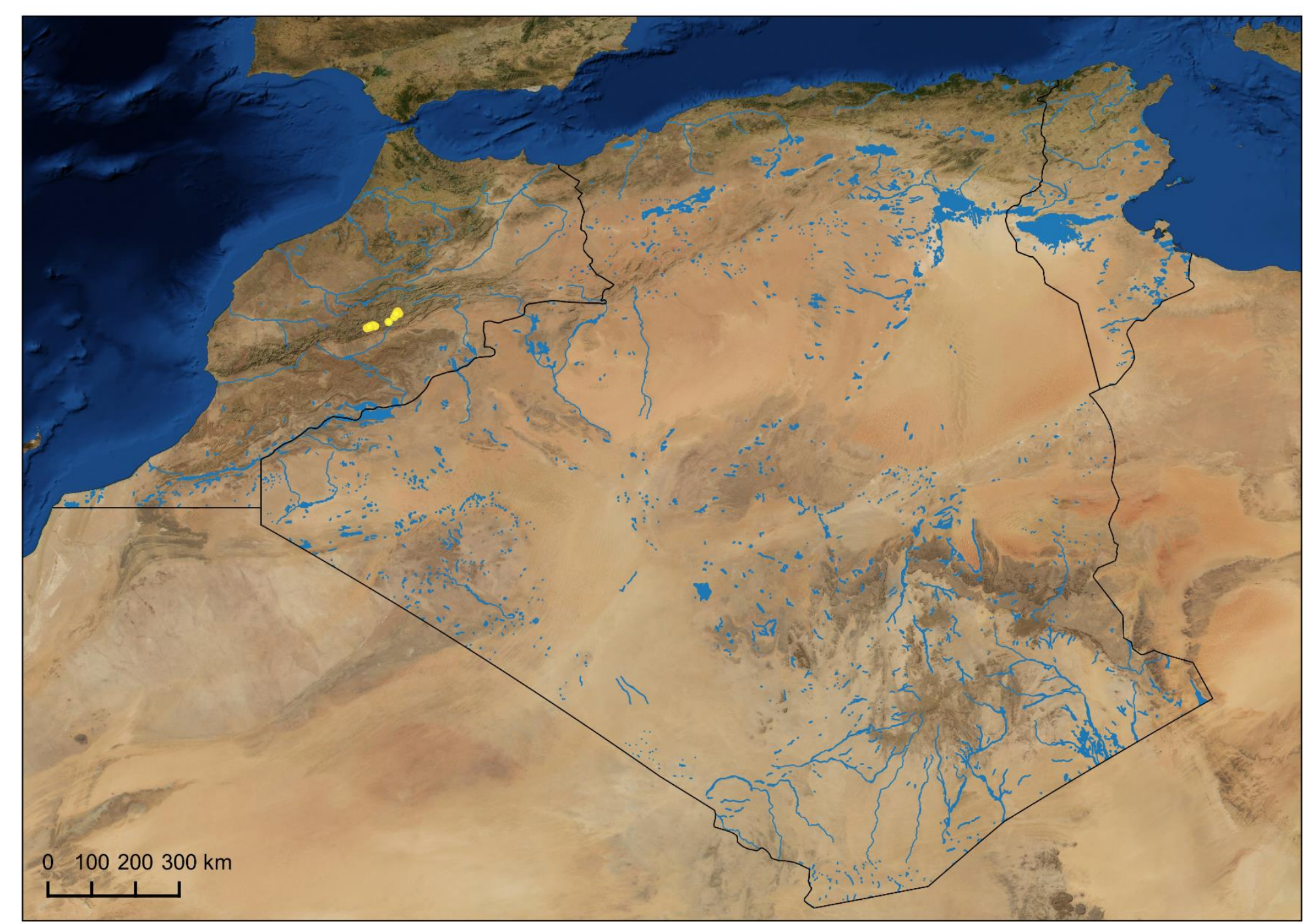

Figure 57: Salmo multipunctata. 


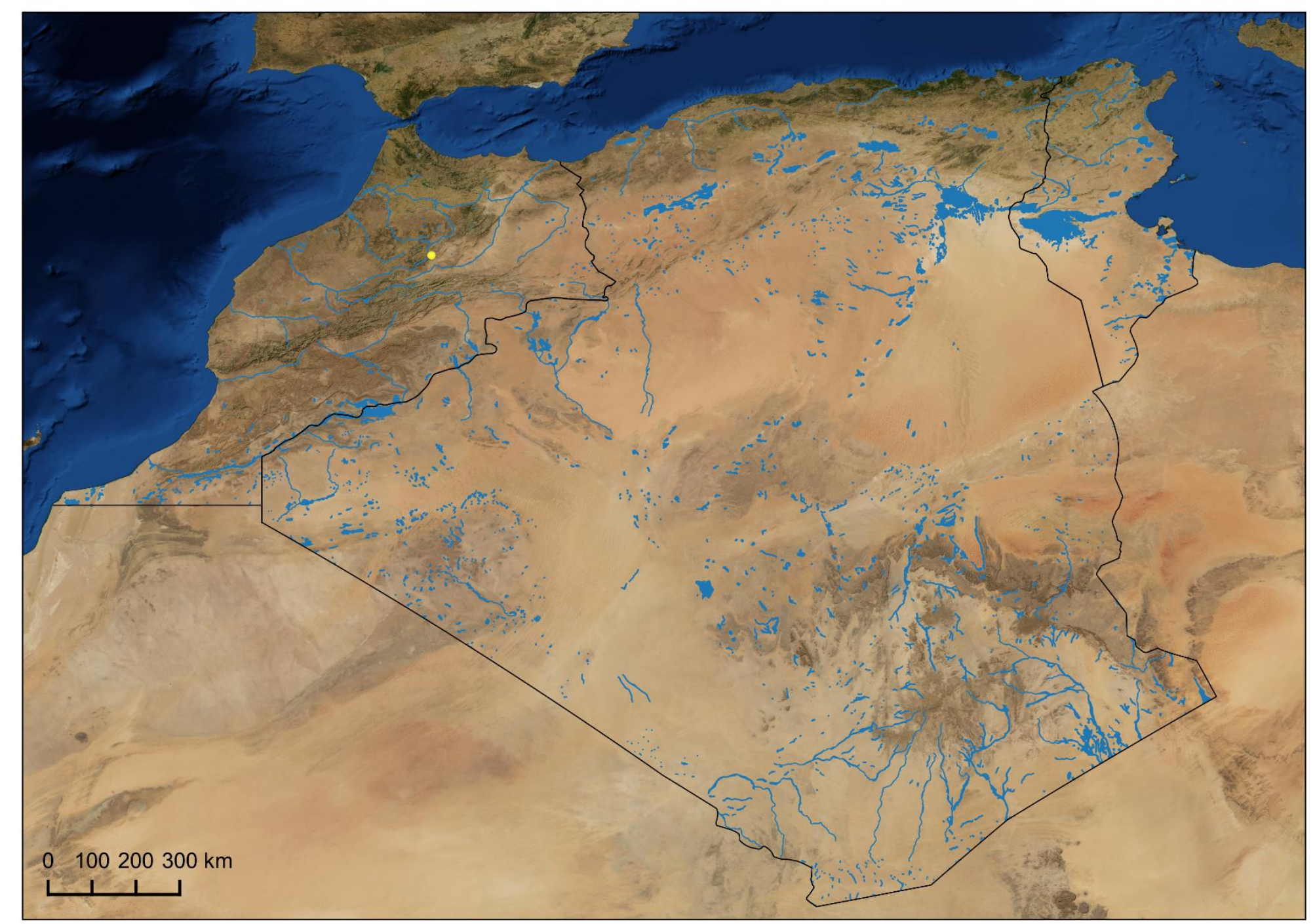

Figure 58: Salmo pallaryi. Extinct since the late 1930s. 


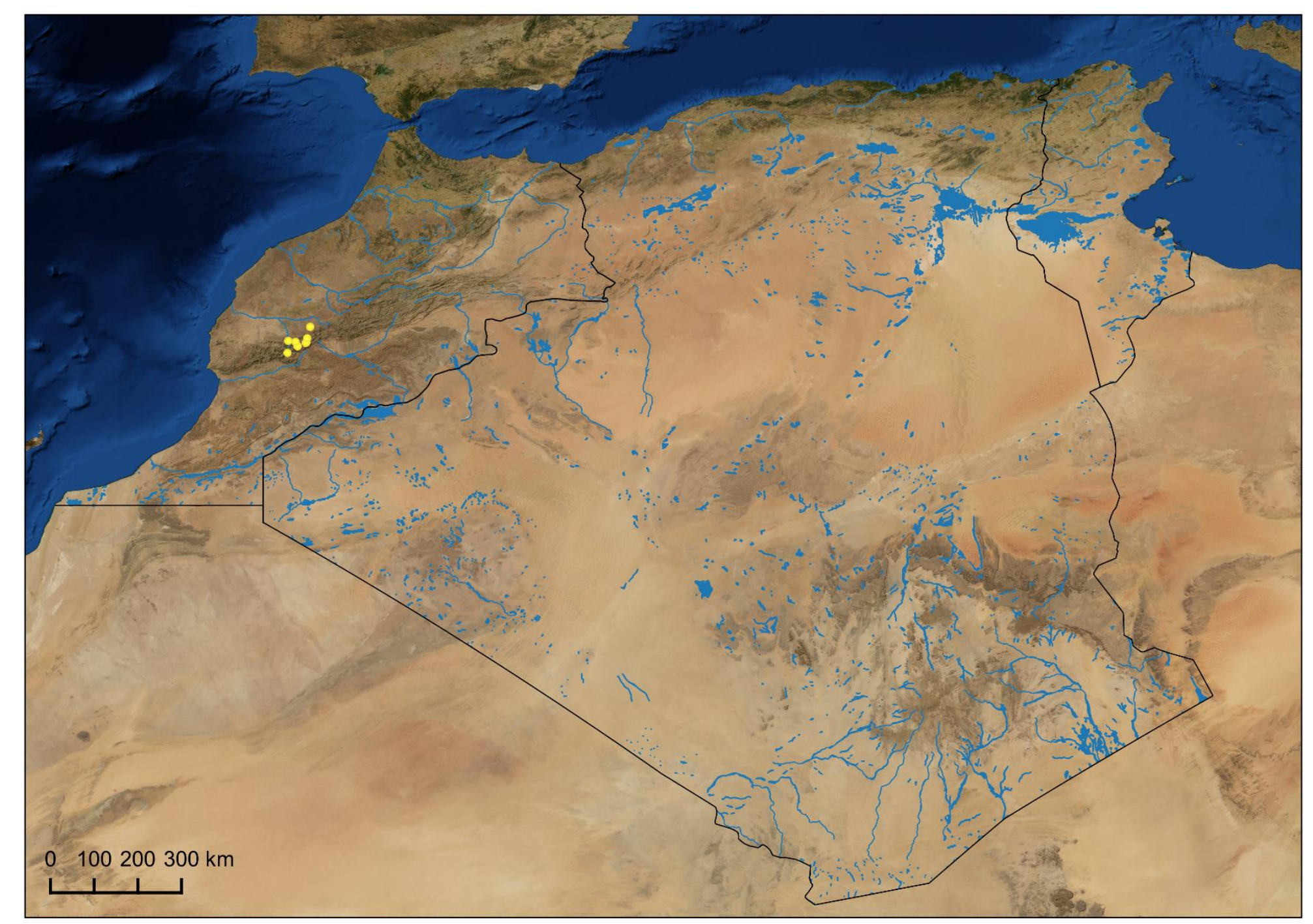

Figure 59: Salmo pellegrini. 


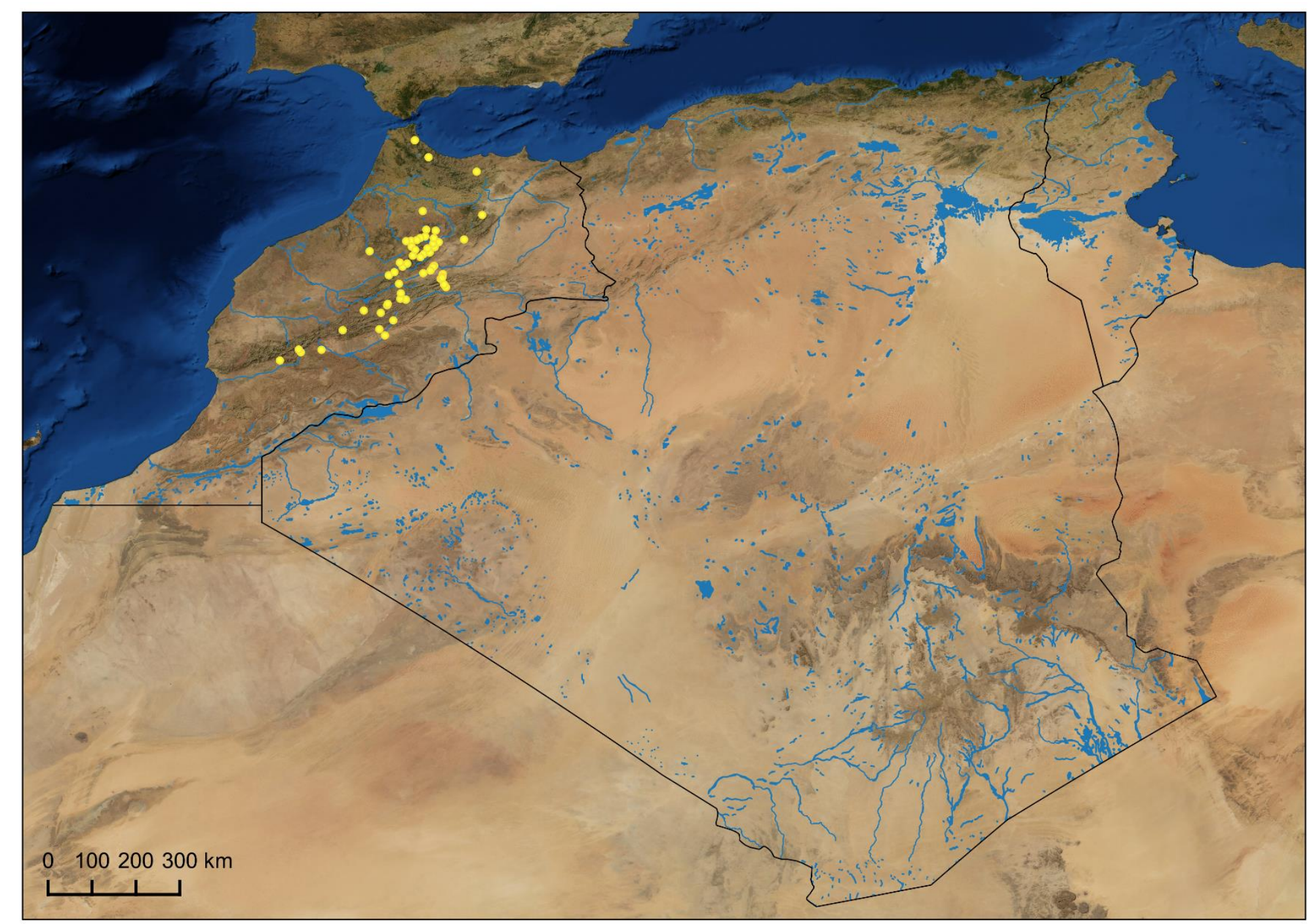

Figure 60: Salmo cf. trutta. 


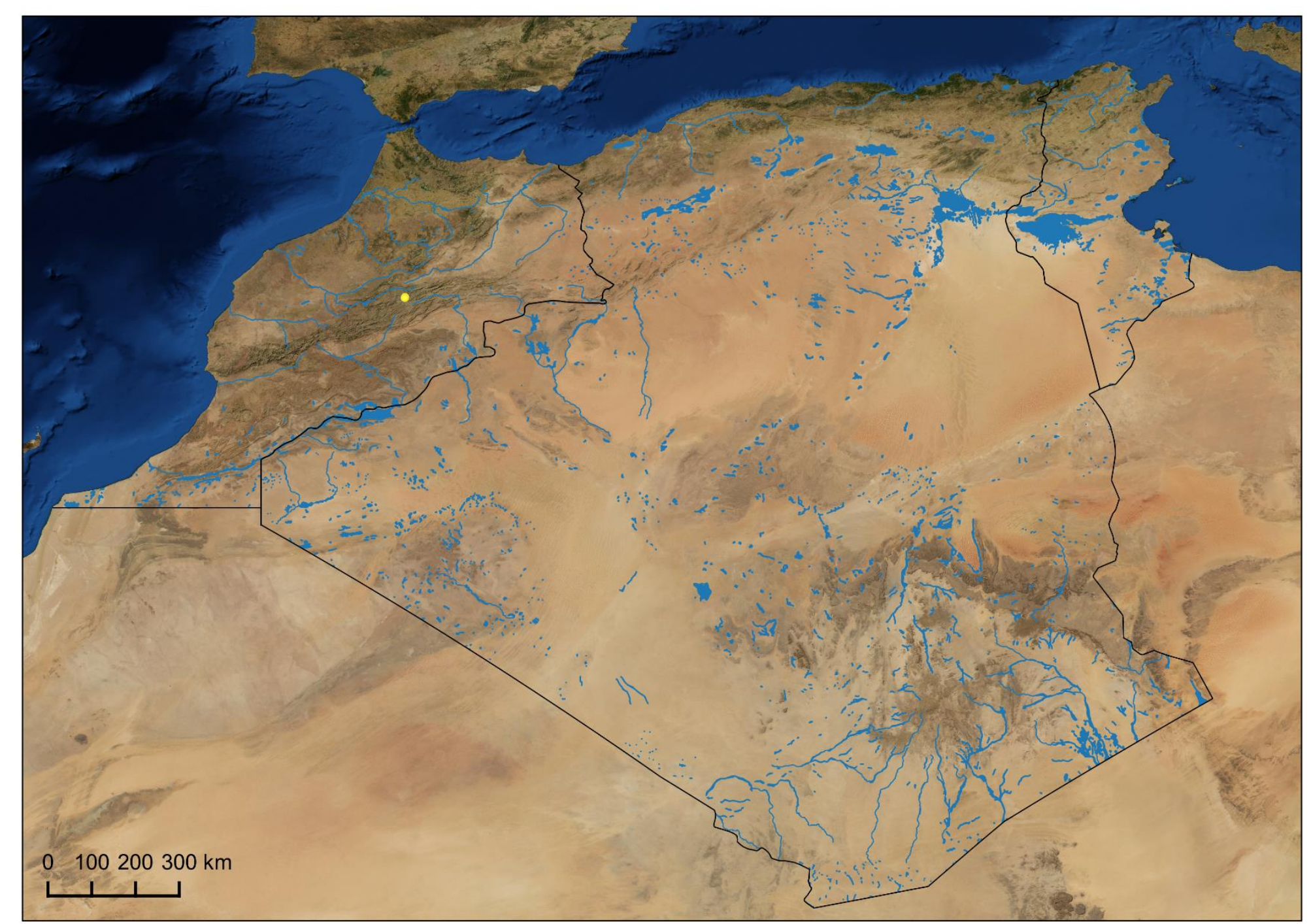

Figure 61: Salmo viridis. 


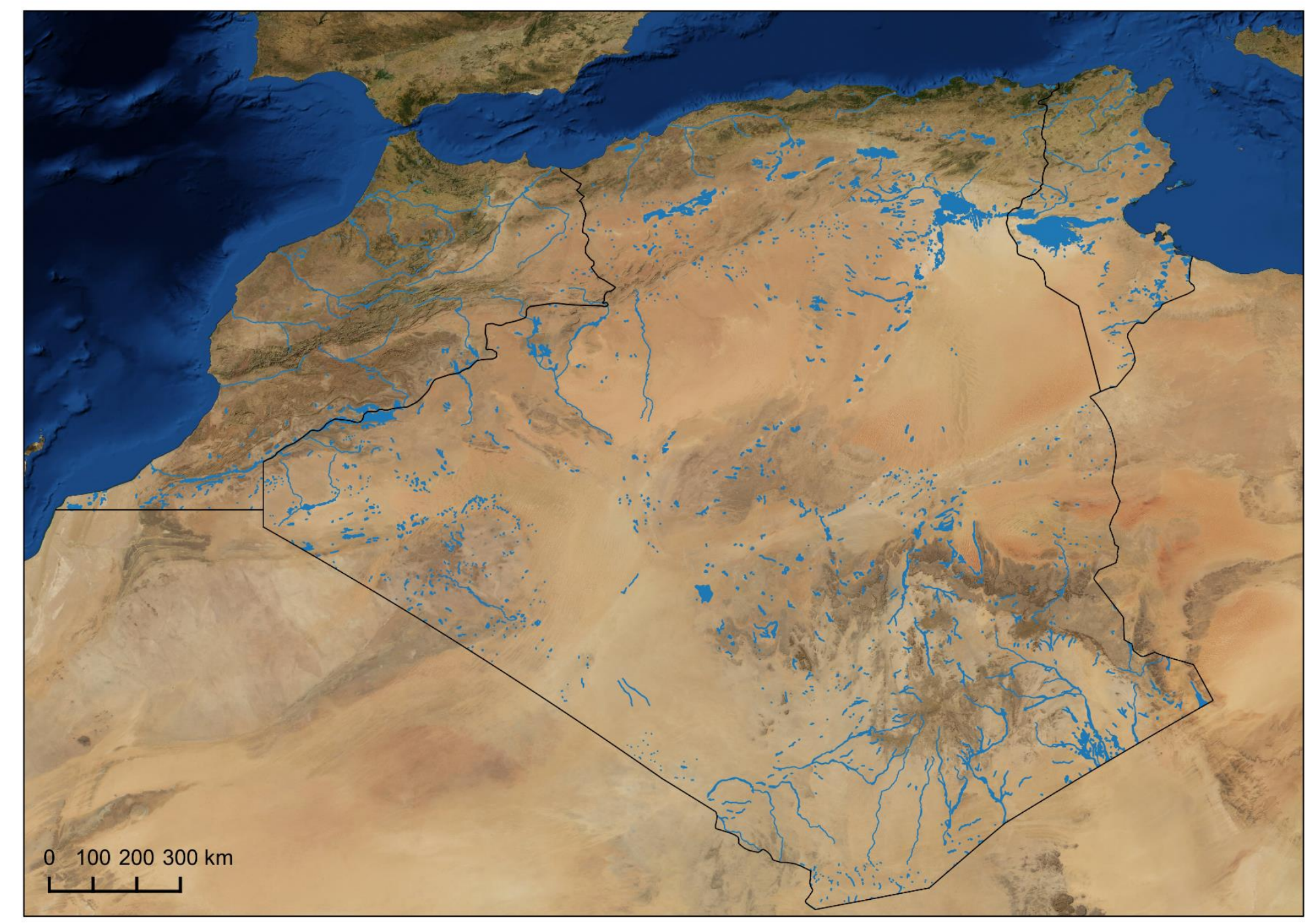

Figure 62: Salvelinus fontinalis. Introduced at a number of locations but no precise site-scale records are available. 


\section{Family: Mugilidae}

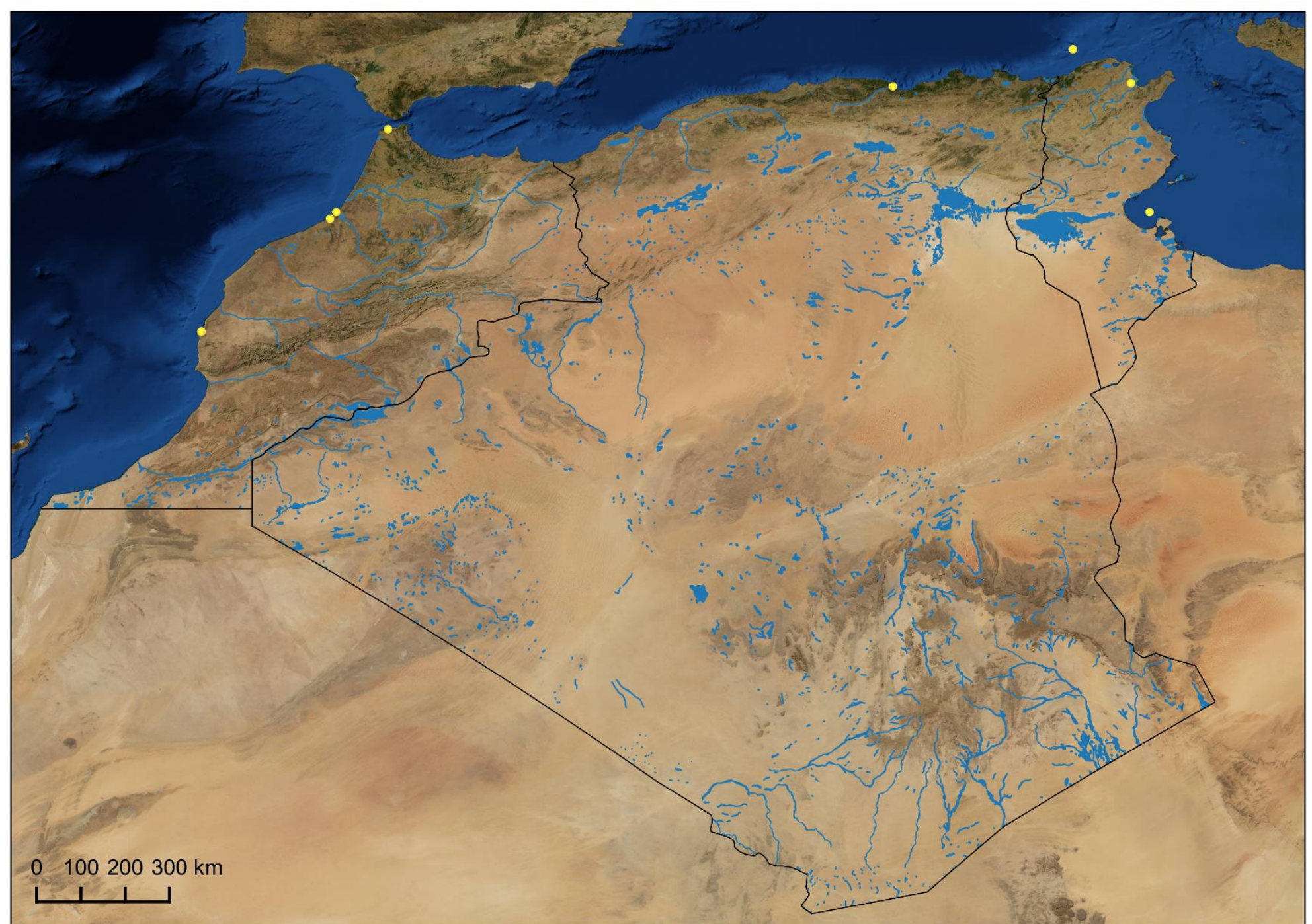

Figure 63: Chelon auratus. Widespread along all coastlines but few precise site-scale records are available. 


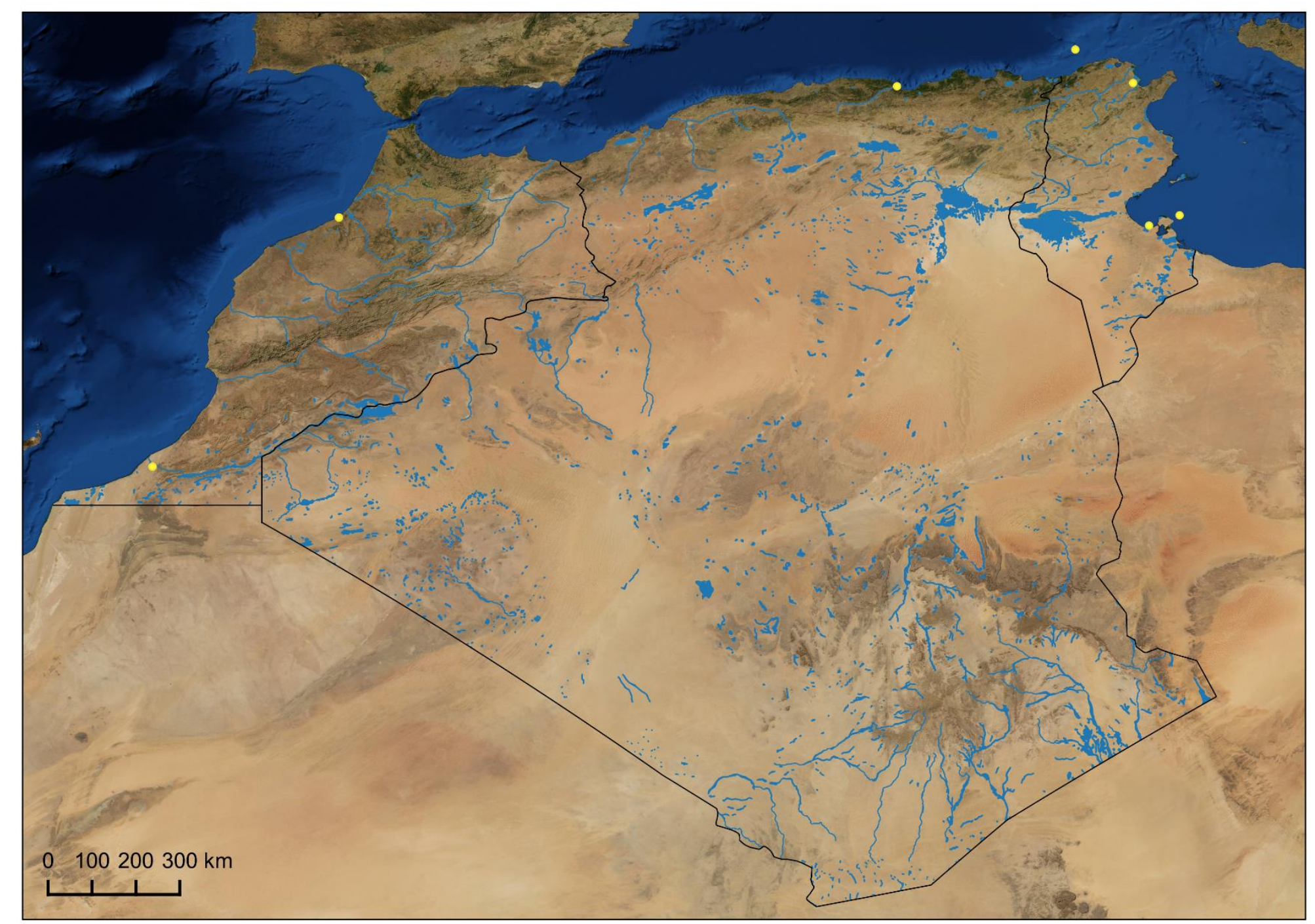

Figure 64: Chelon labrosus. Widespread along all coastlines but few precise site-scale records are available. 


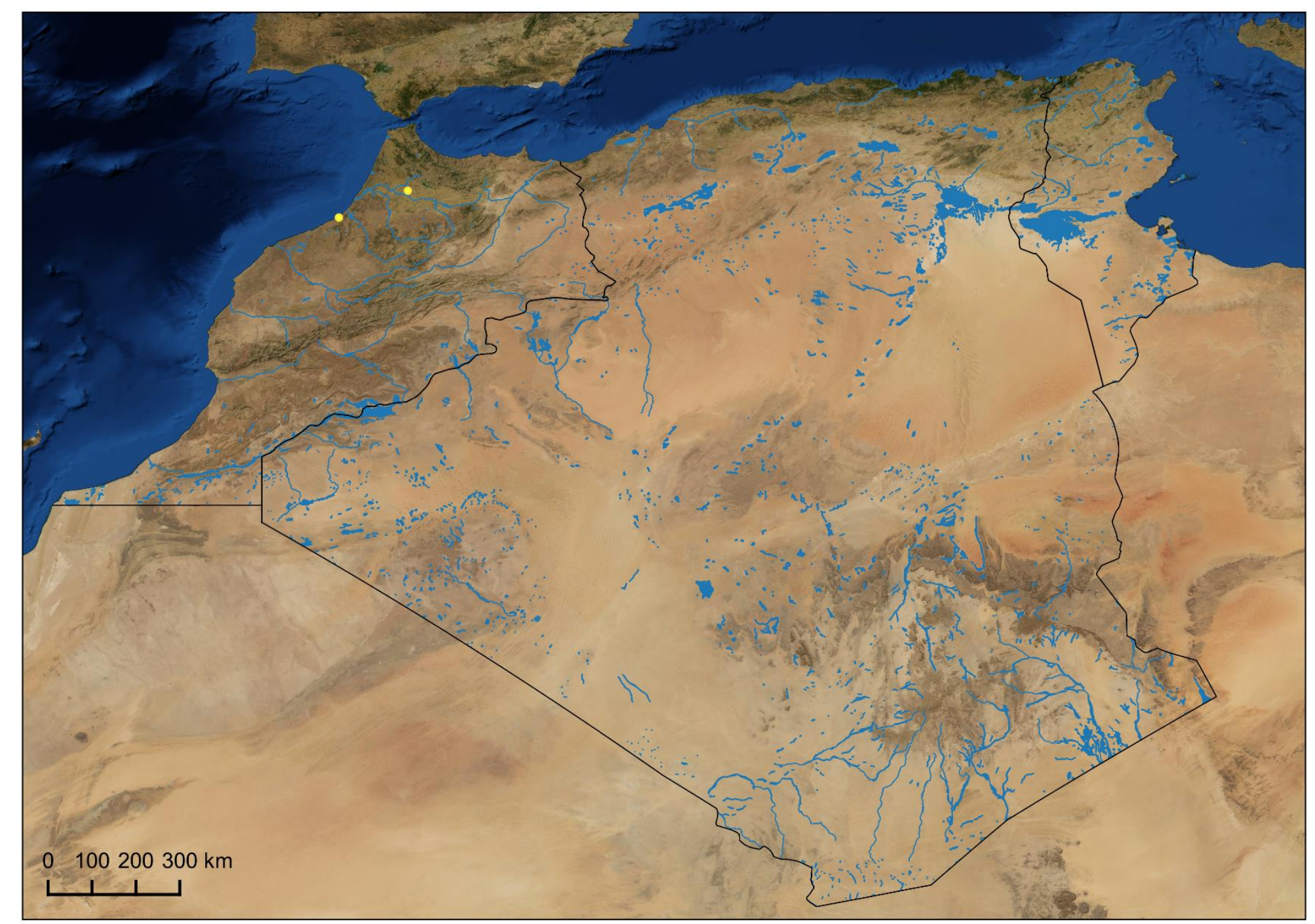

Figure 65: Chelon ramada. Widespread along all coastlines but few precise site-scale records are available. 


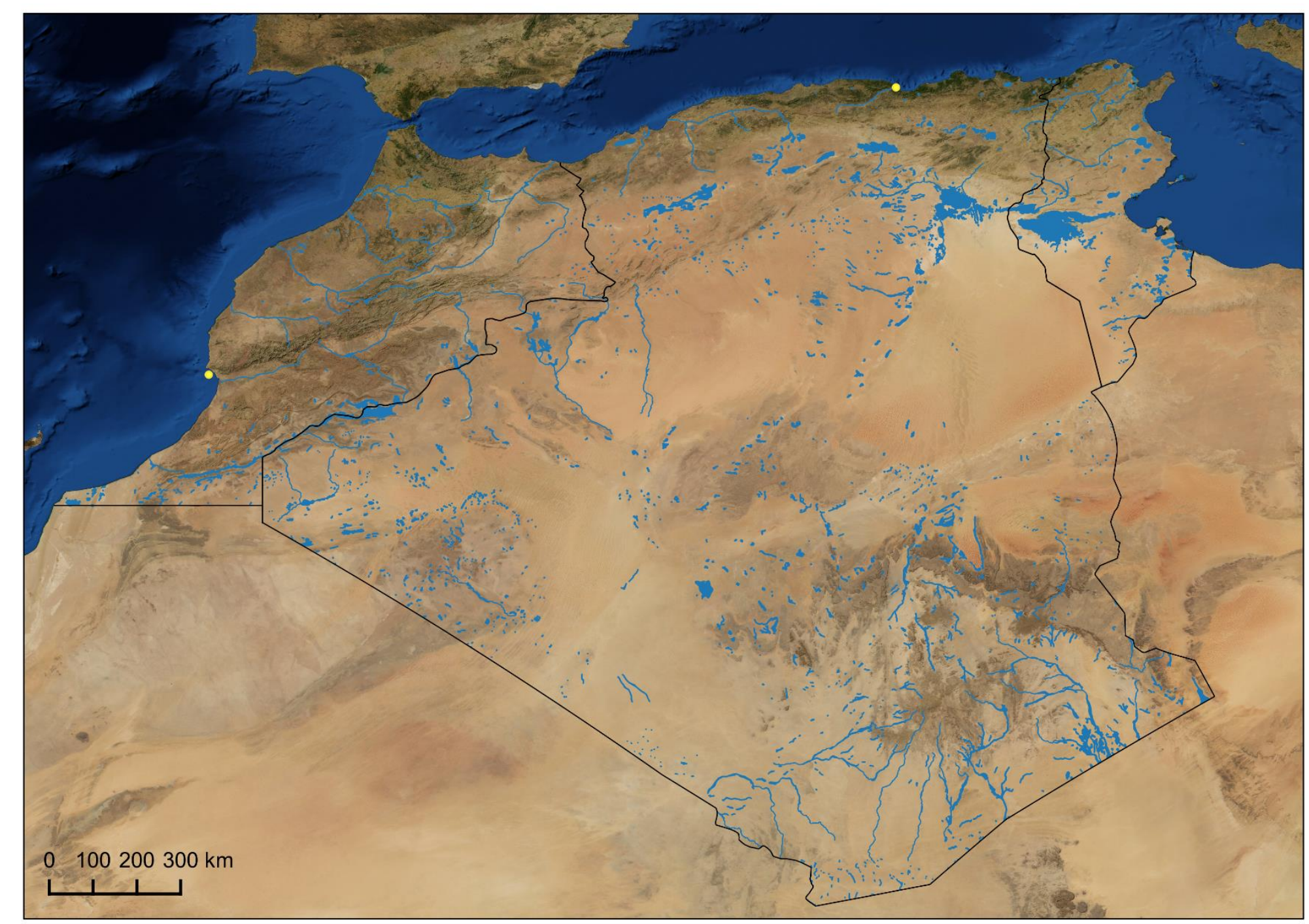

Figure 66: Chelon saliens. Widespread along all coastlines but few precise site-scale records are available. 


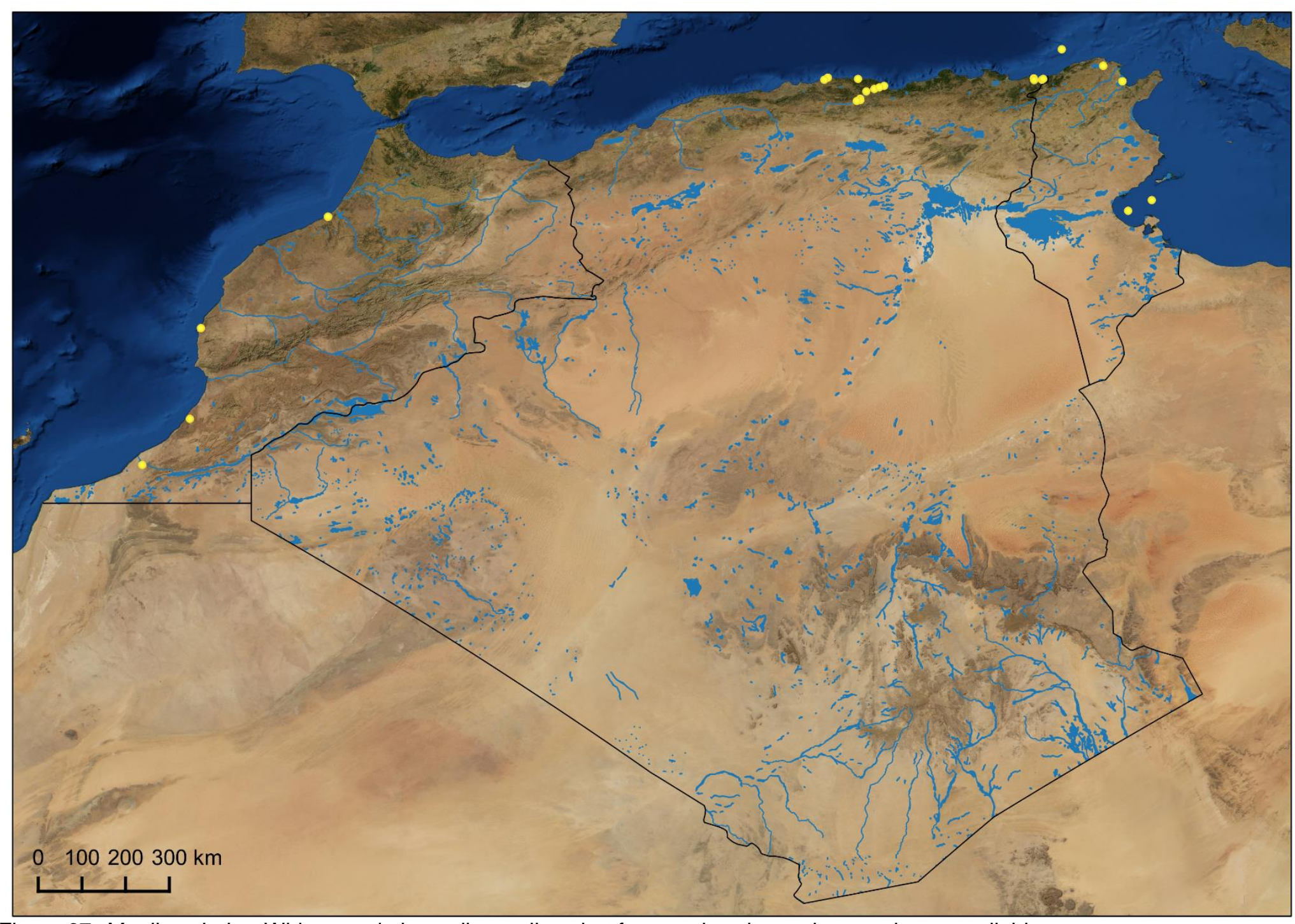

Figure 67: Mugil cephalus. Widespread along all coastlines but few precise site-scale records are available. 


\section{Family: Atherinidae}

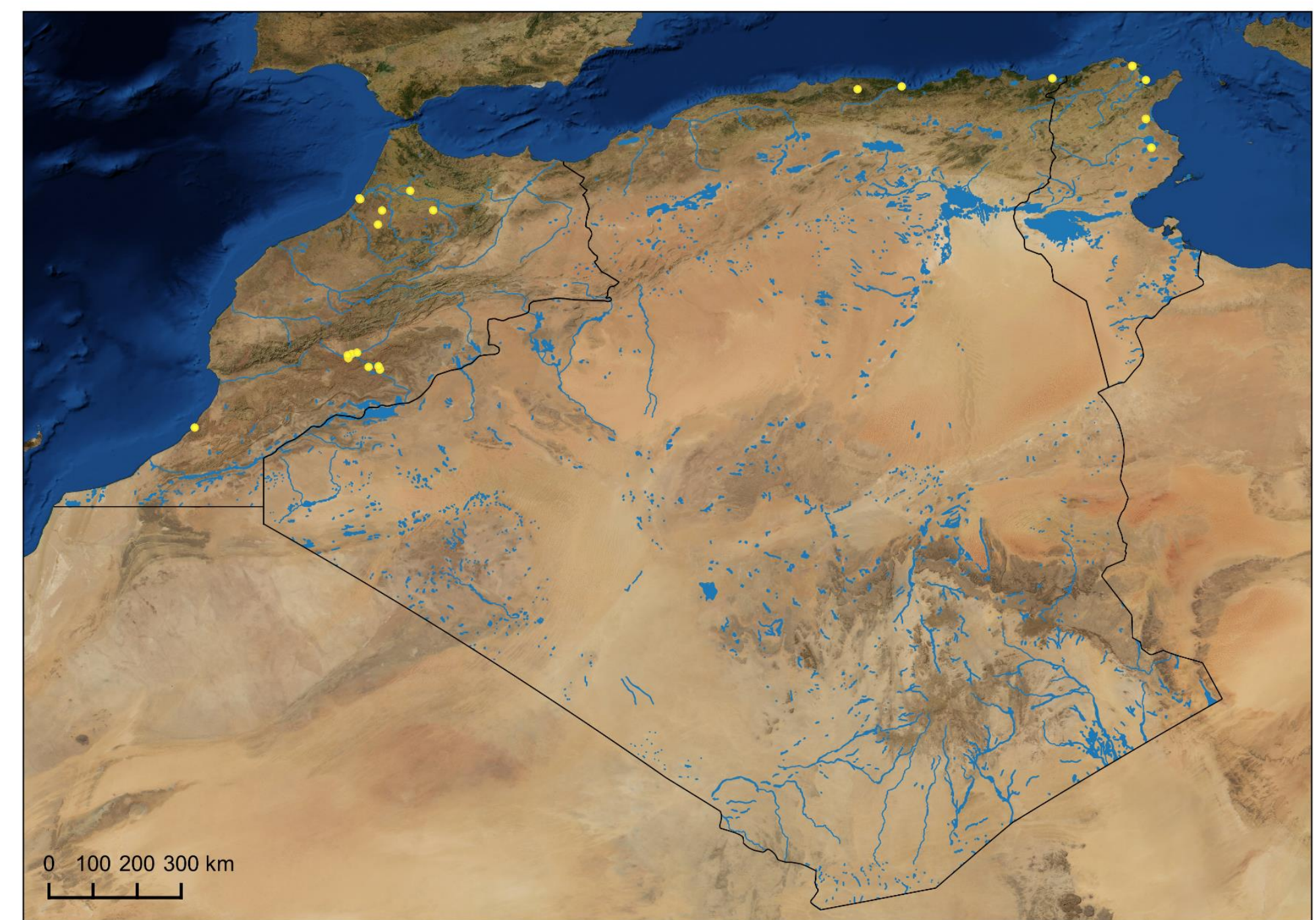

Figure 68: Atherina boyeri. Widespread along all coastlines and introduced in some river drainages, e.g., the Draa (Morocco). 


\section{Family: Aphaniidae}

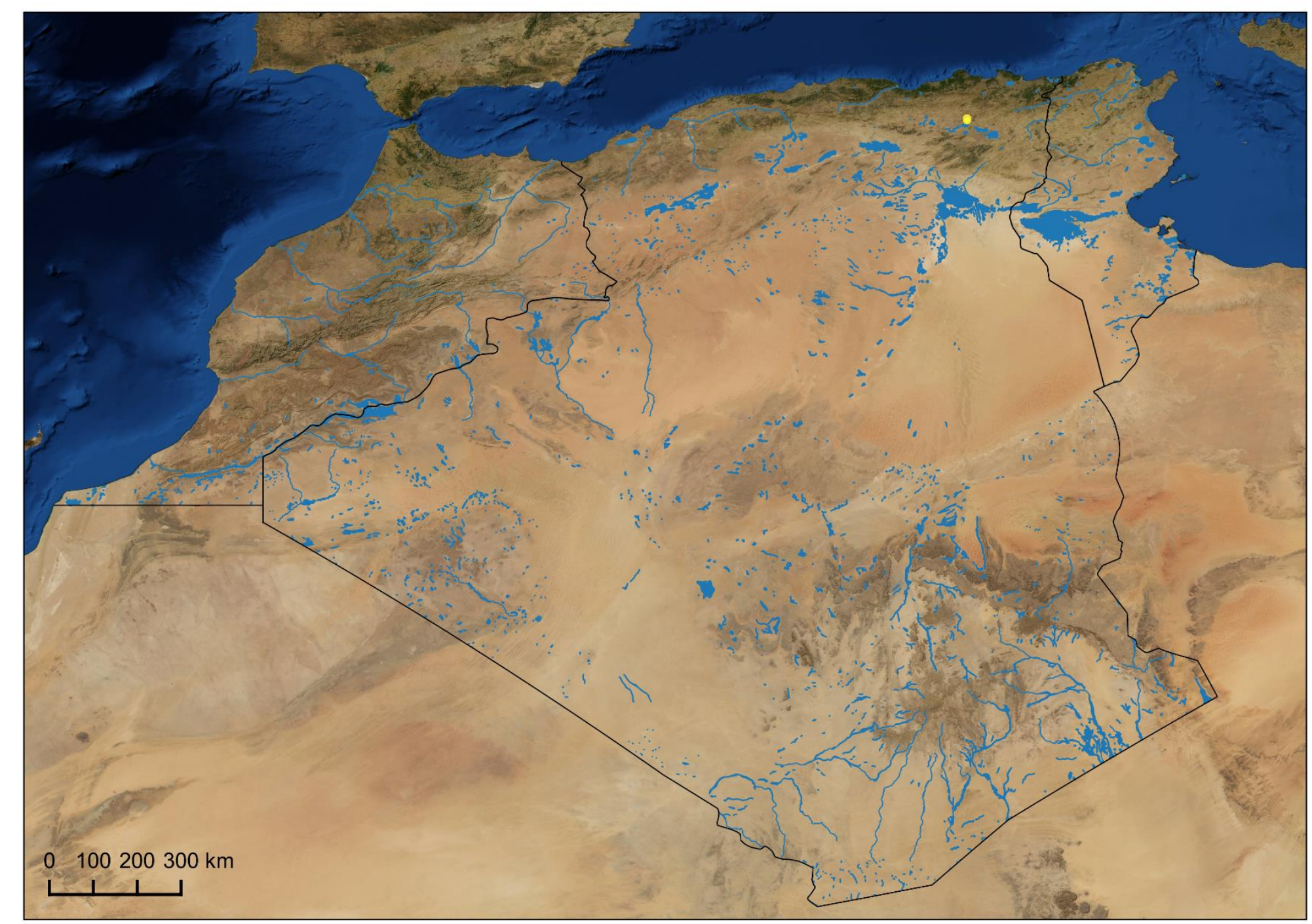

Figure 69: Aphanius apodus. Last recorded in the wild during 1990. 


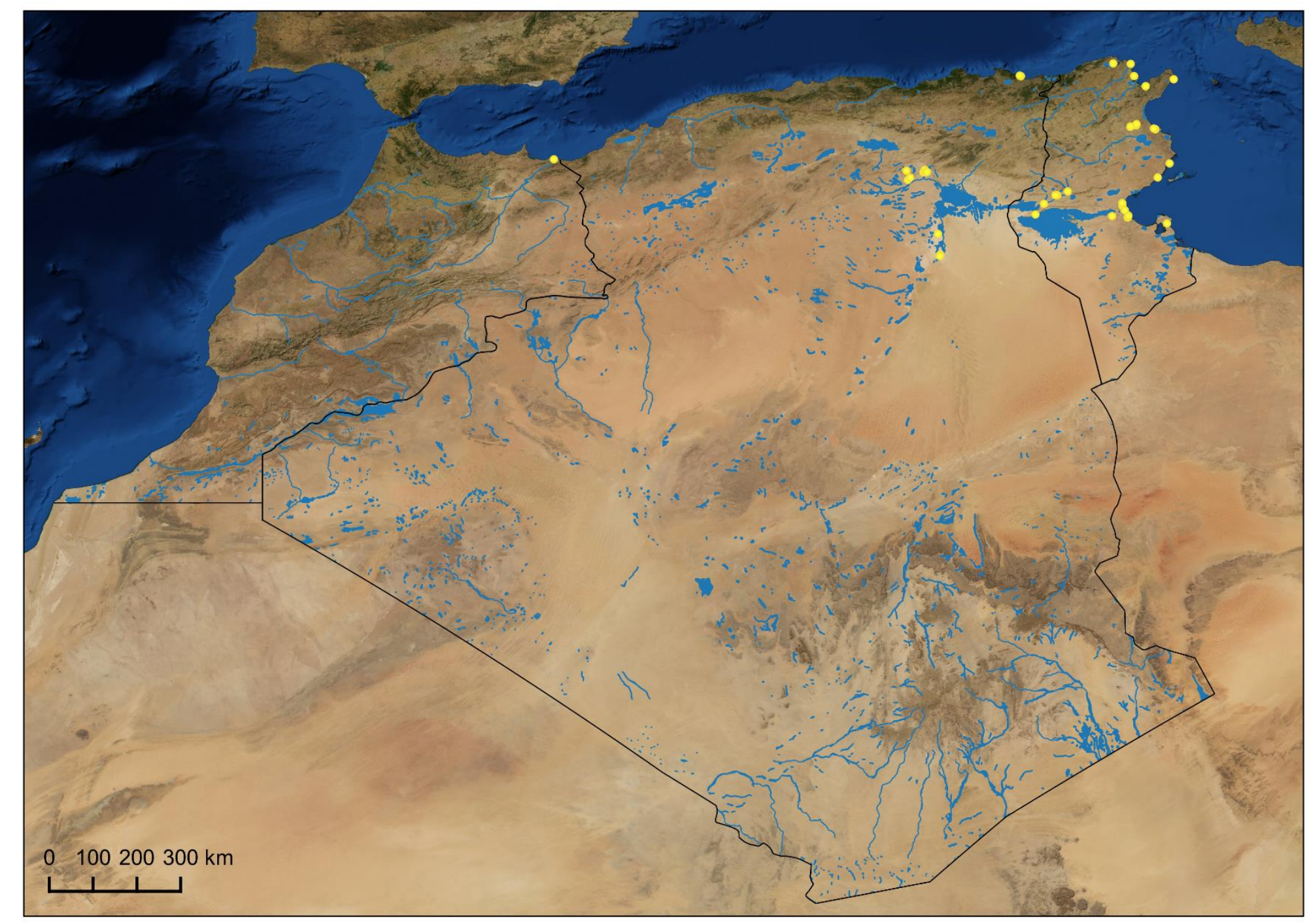

Figure 70: Aphanius fasciatus. Expected to be more widespread along the Algerian coast, but few site-scale records exist. 


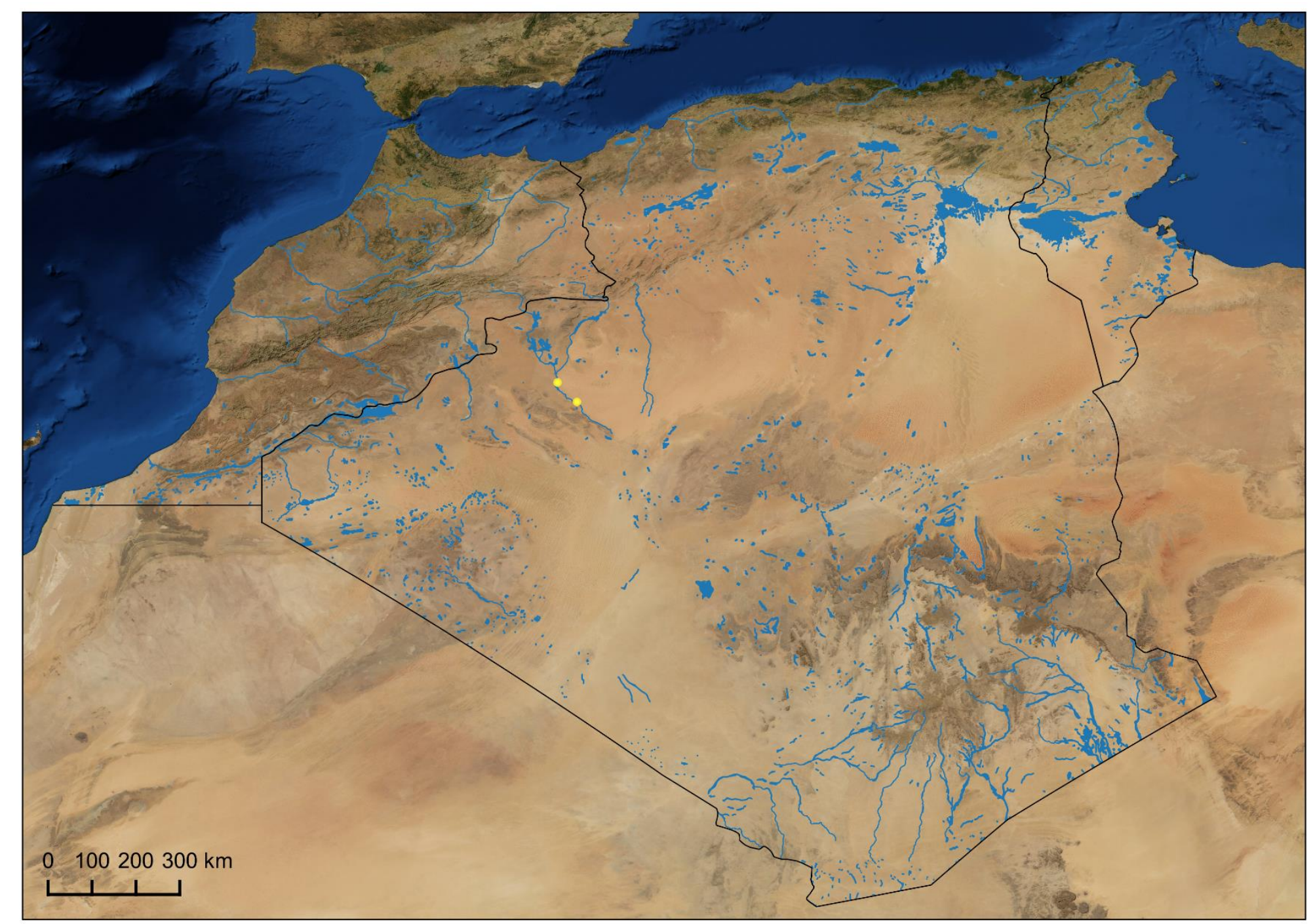

Figure 71: Aphanius saourensis. Last recorded in the wild during 2004. 


\section{Family: Poeciliidae}

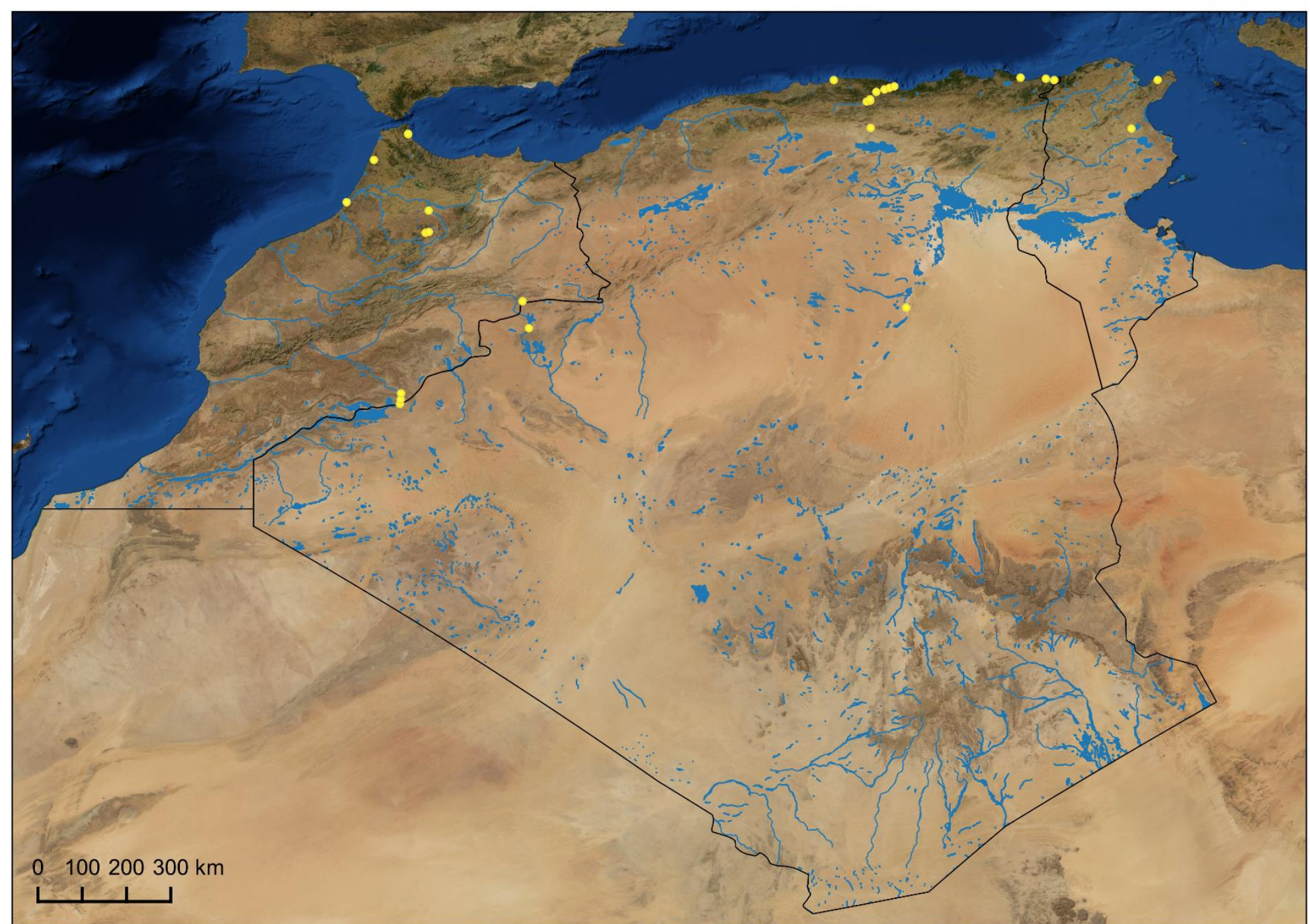

Figure 72: Gambusia holbrooki. Widespread in the region but few precise site-scale records are available. 


\section{Family: Syngnathidae}

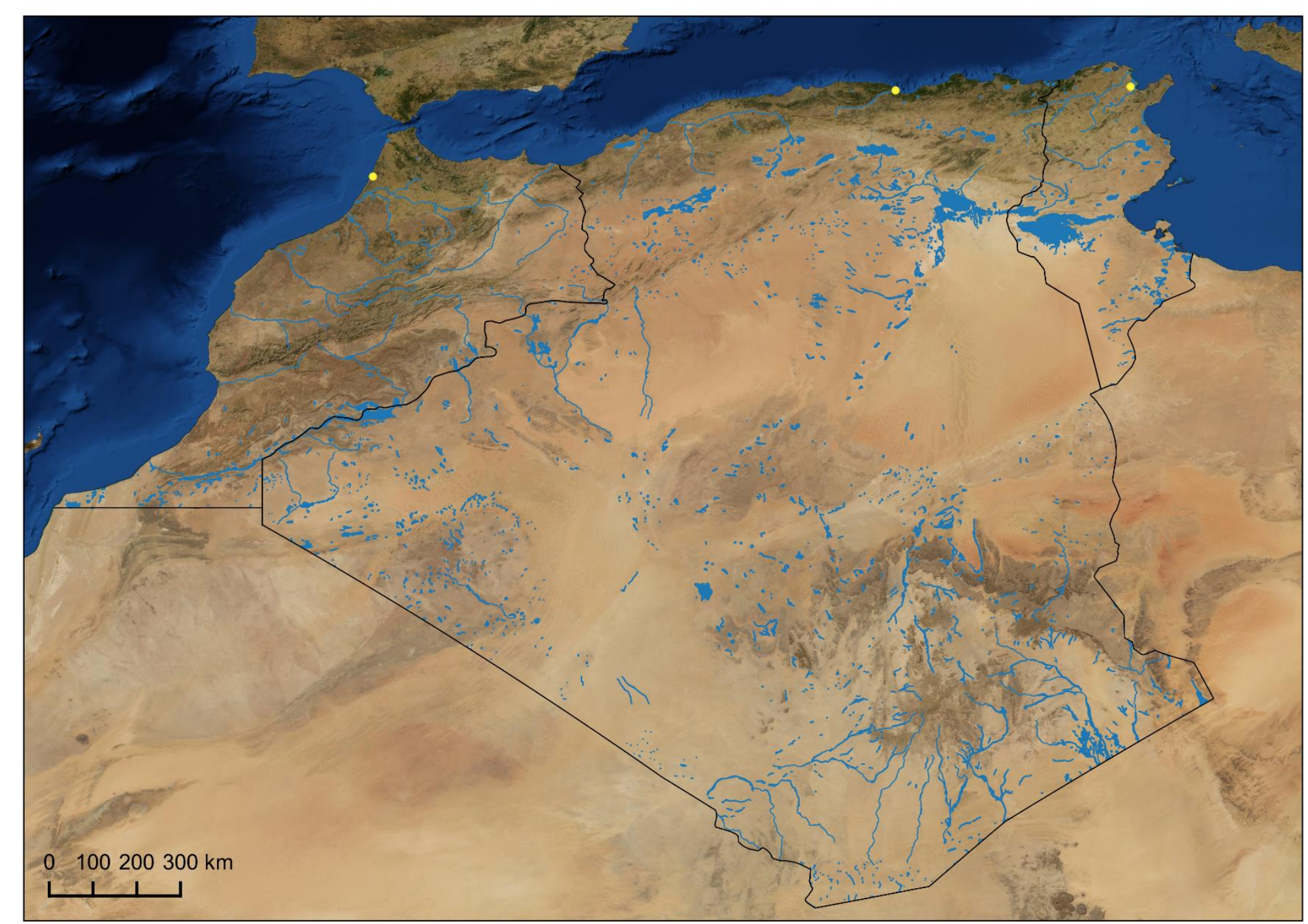

Figure 73: Syngnathus abaster. Widespread along all coastlines but few site-scale records are available. 


\section{Family: Moronidae}

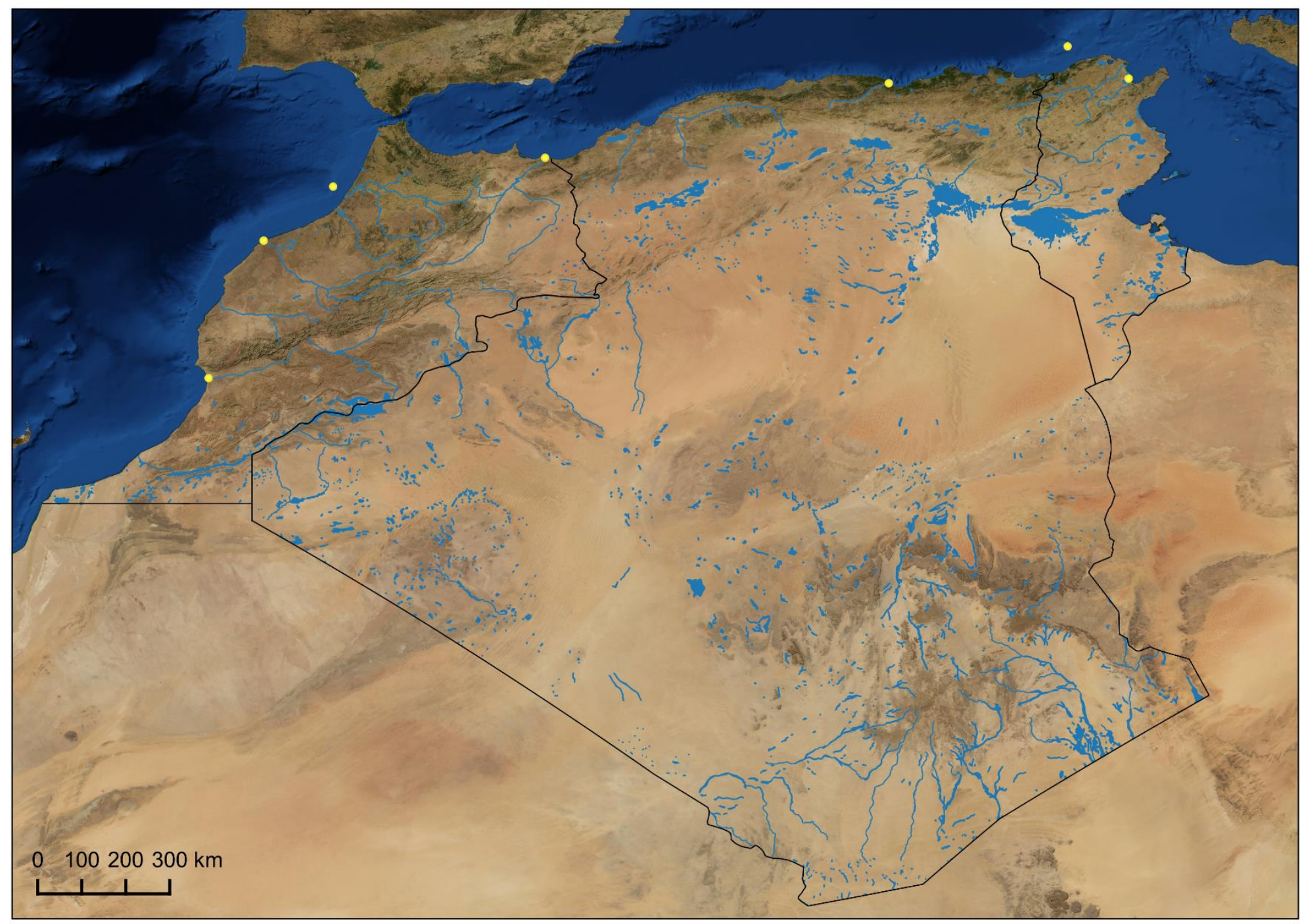

Figure 74: Dicentrarchus labrax. Widespread along all coastlines but few site-scale records are available. 


\section{Family: Centrarchidae}

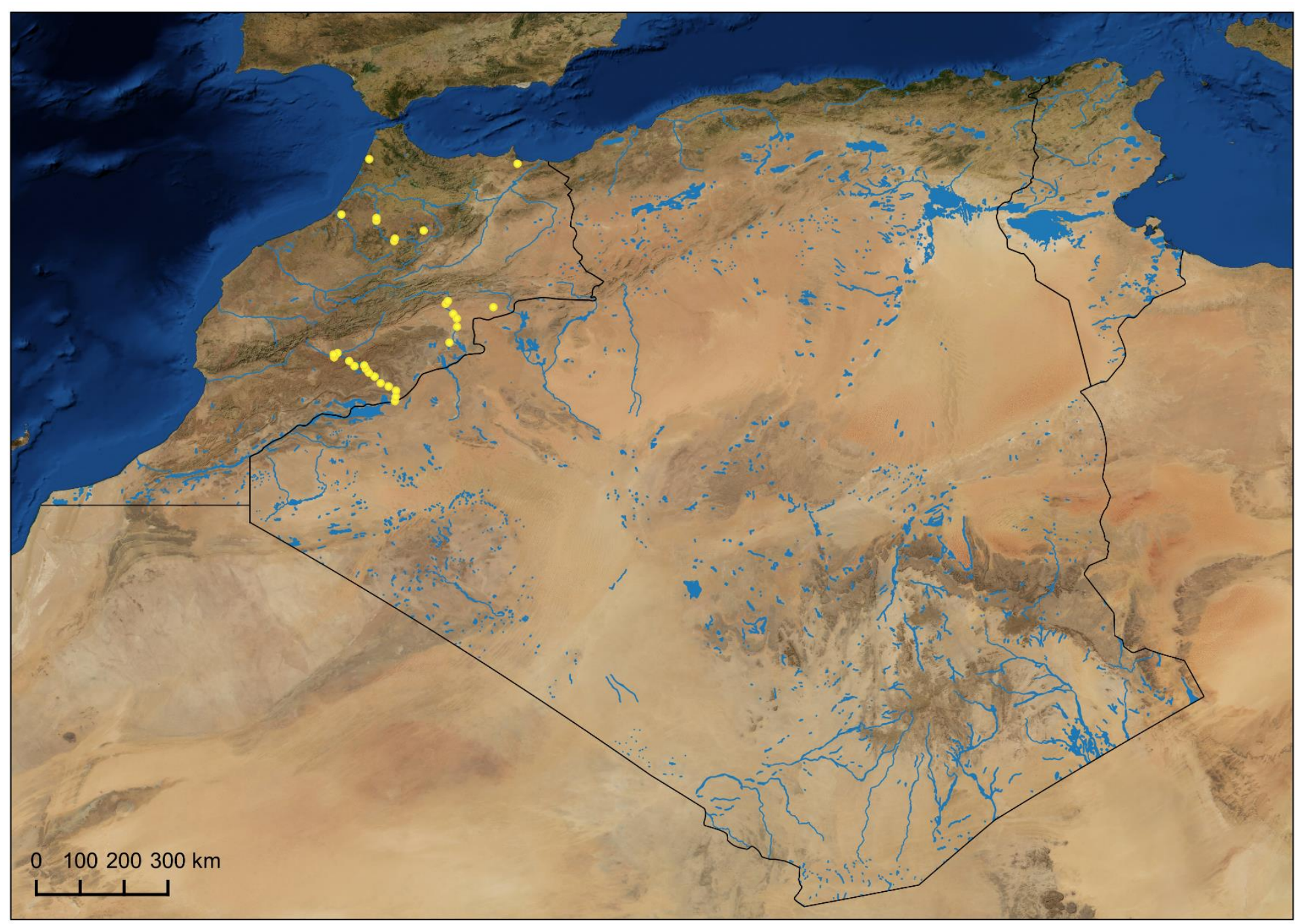

Figure 75: Lepomis gibbosus. Widespread throughout much of the region but few site-scale records are available. 


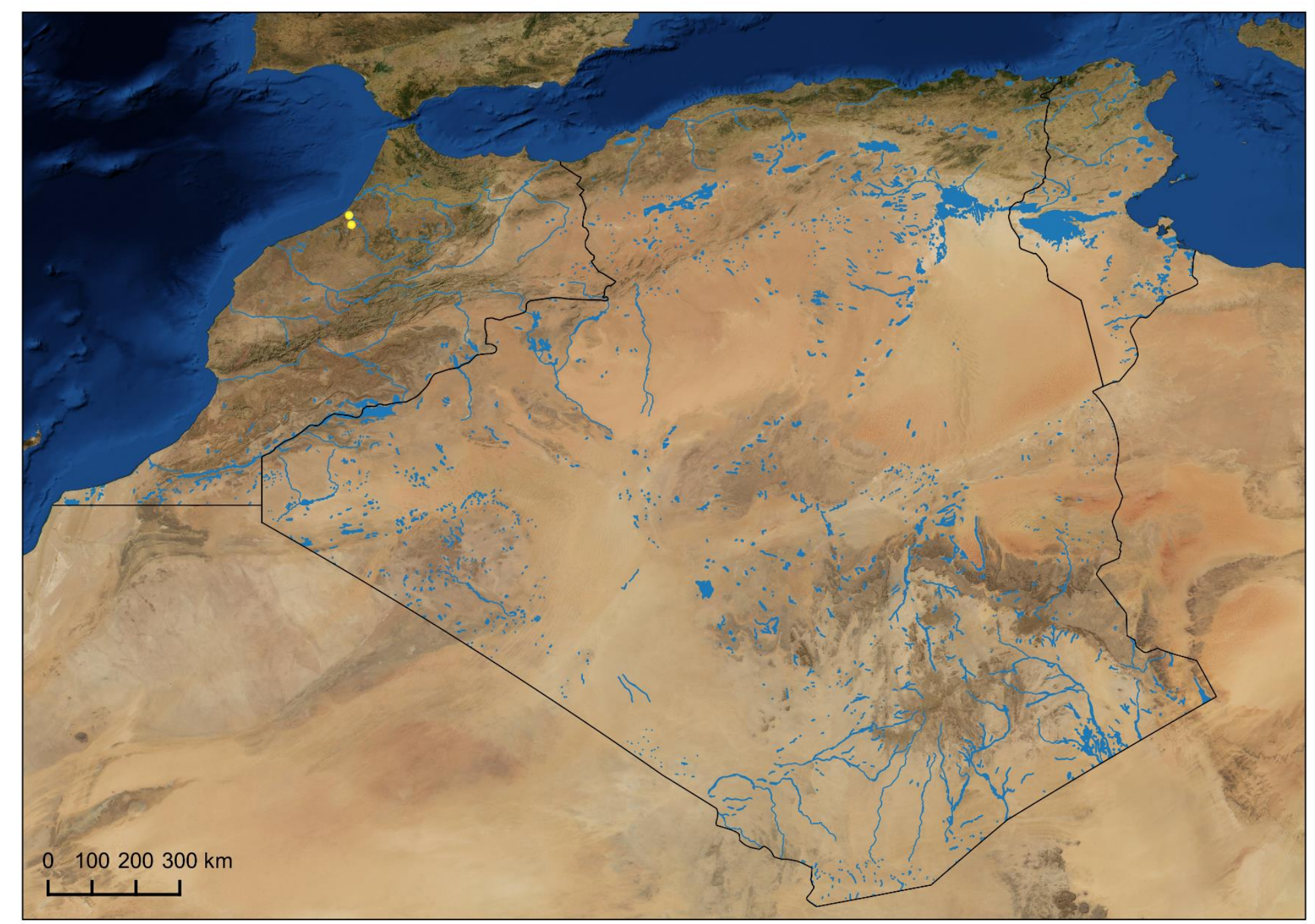

Figure 76: Lepomis macrochirus. Probably more widespread in Morocco but few site-scale records are available. 


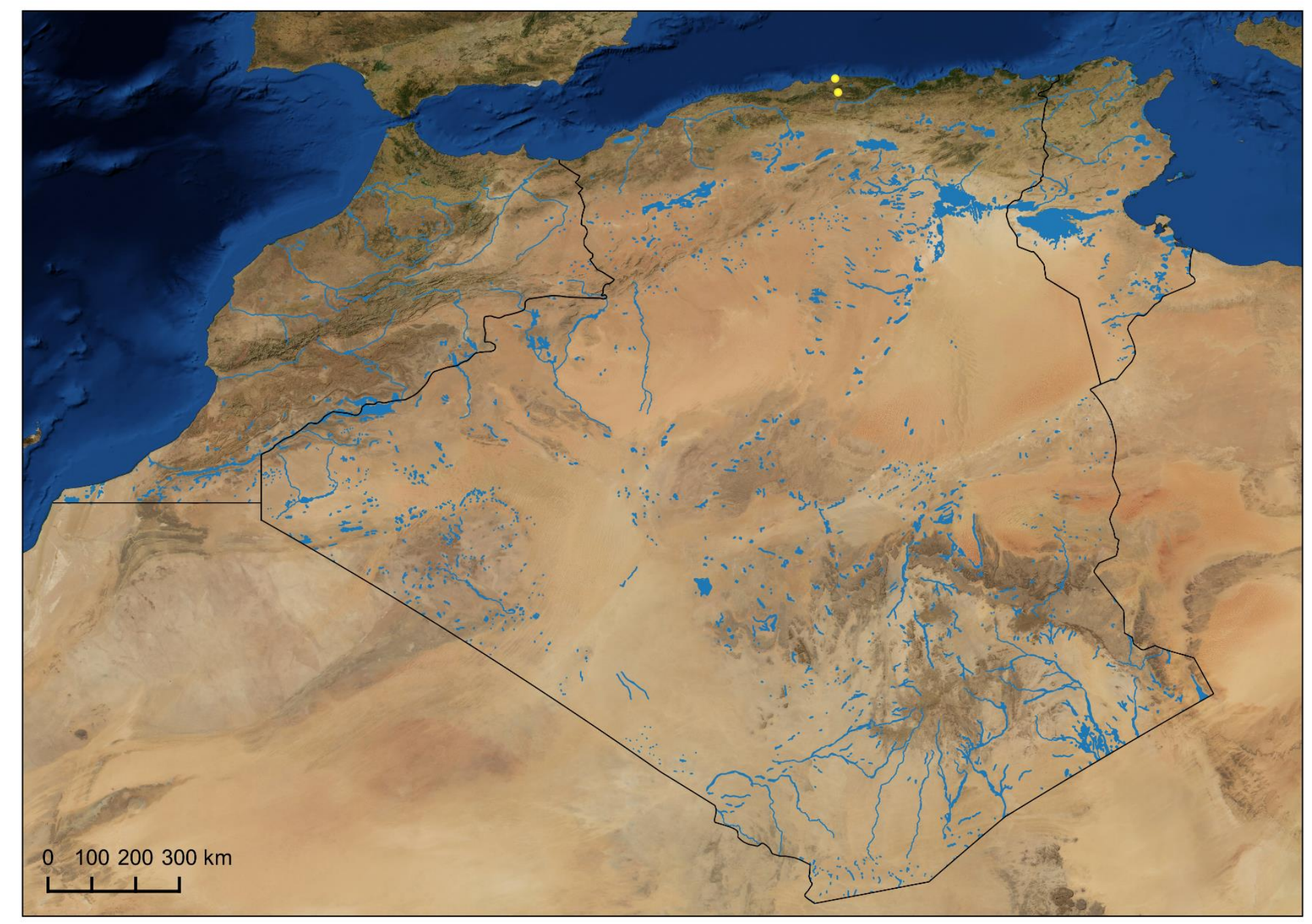

Figure 77: Micropterus salmoides. Recently recorded in the region for the first time and may occur elsewhere. 


\section{Family: Cichlidae}

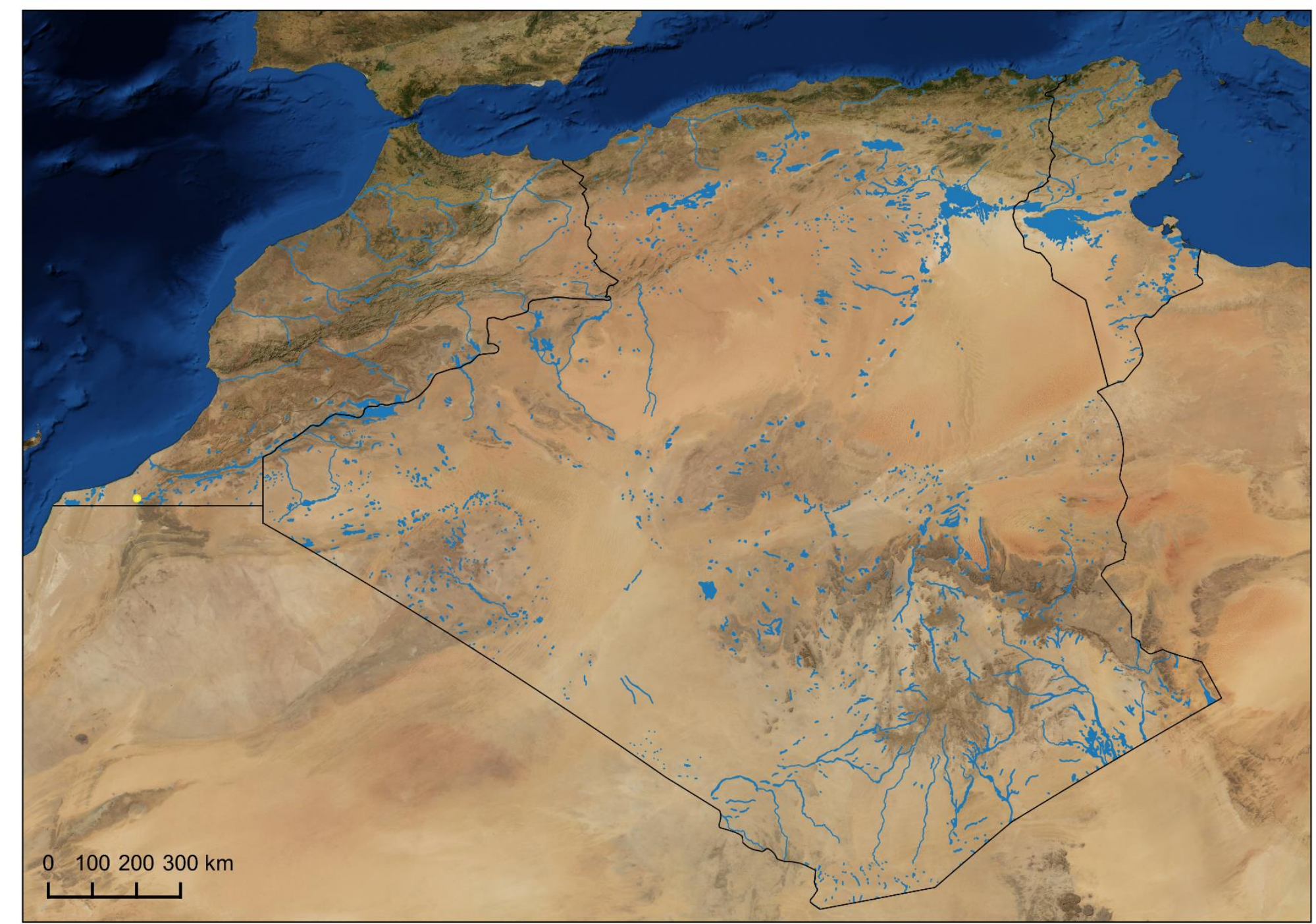

Figure 78: Coptodon polycentra. Likely to be more widespread in southwestern Morocco. 


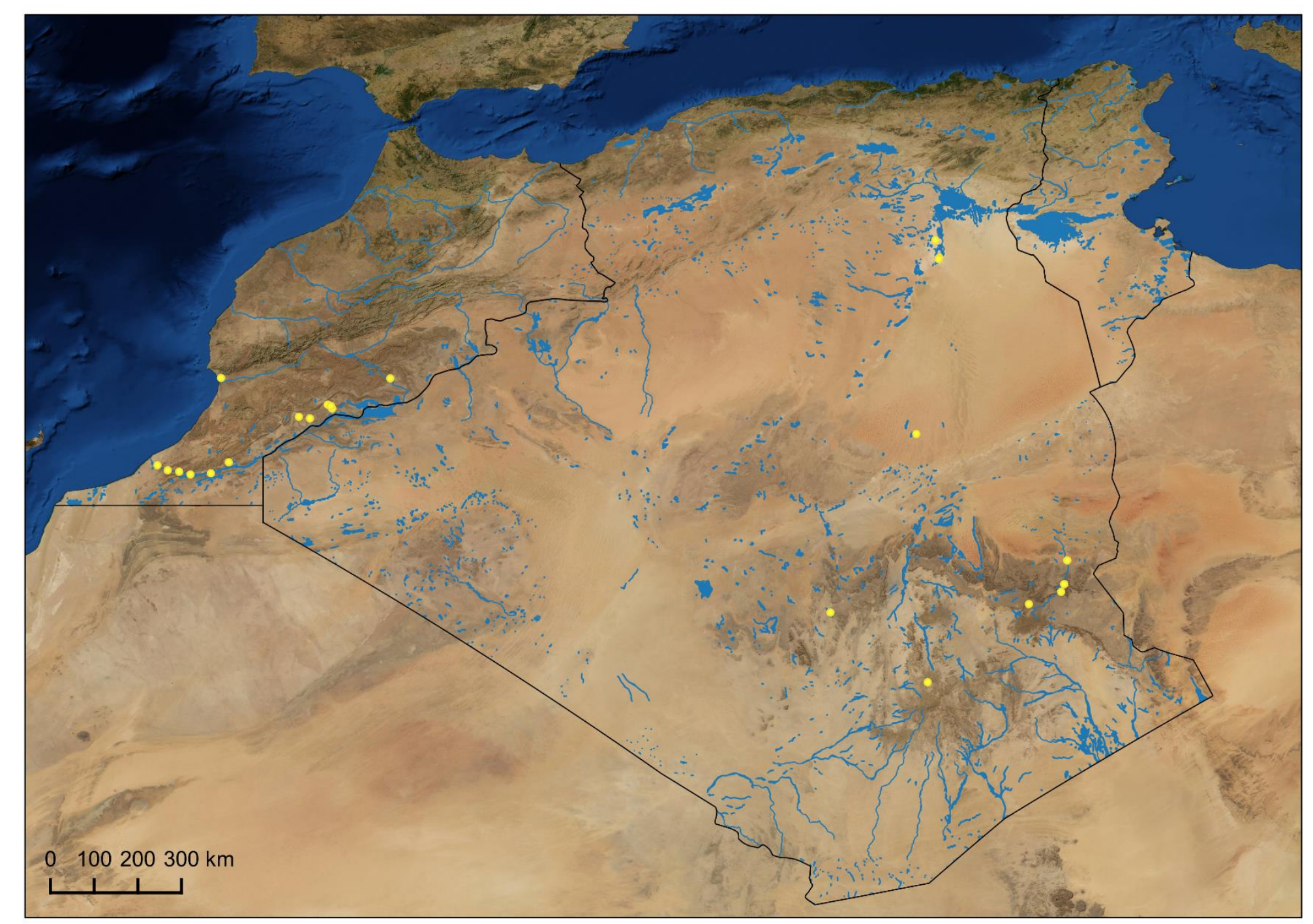

Figure 79: Coptodon zillii. 


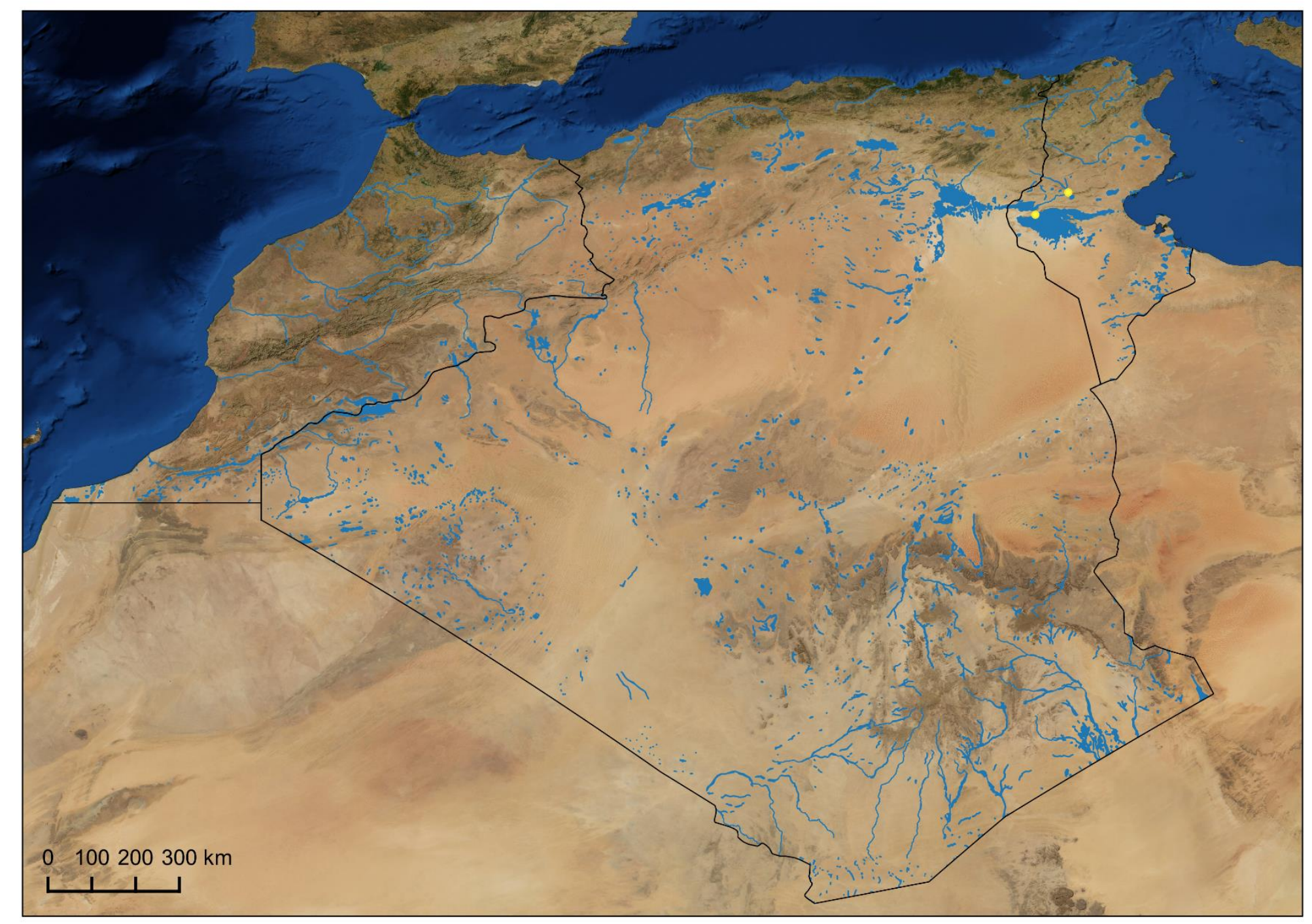

Figure 80: Haplochromis desfontainii. 


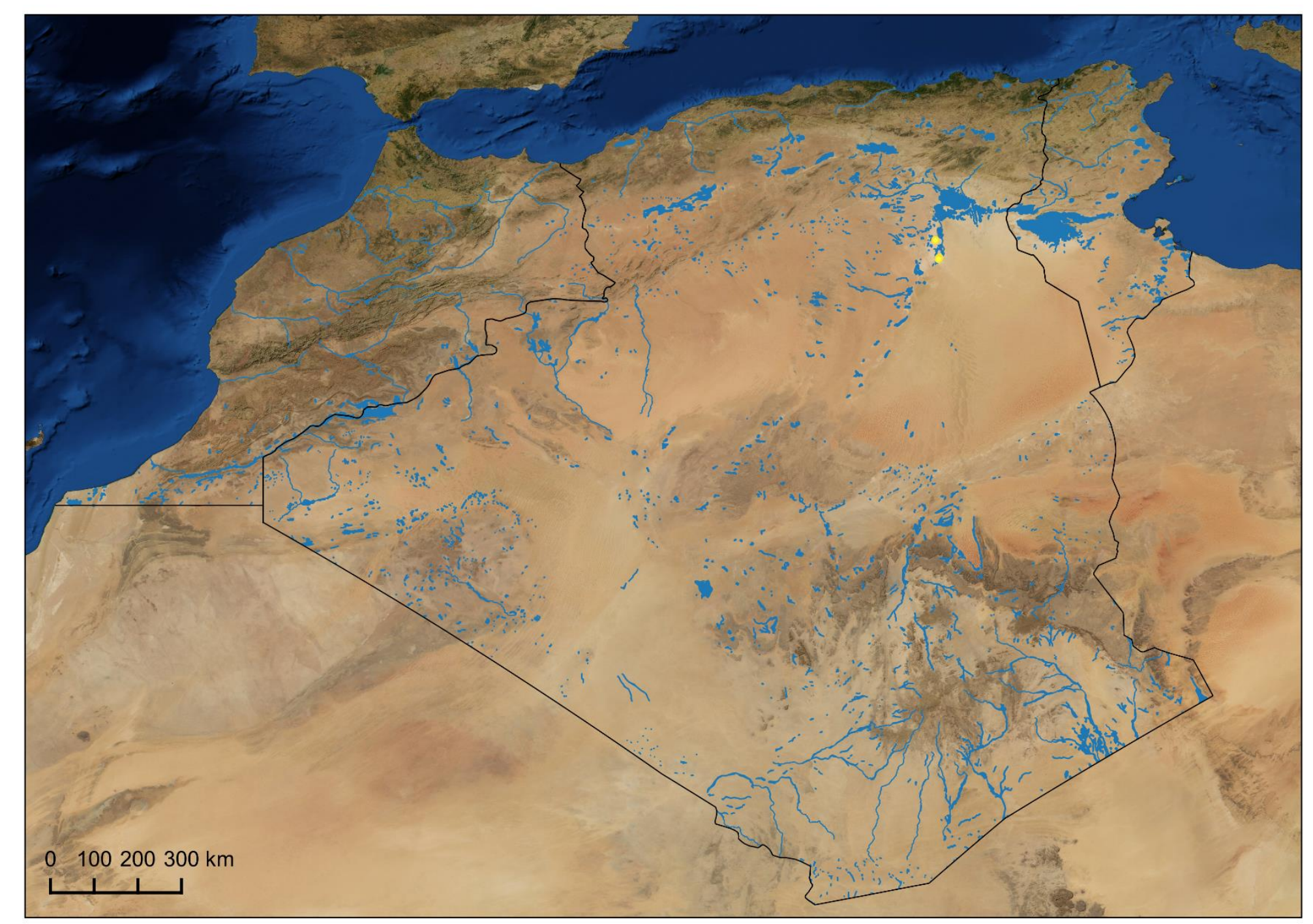

Figure 81: Hemichromis guttatus. 


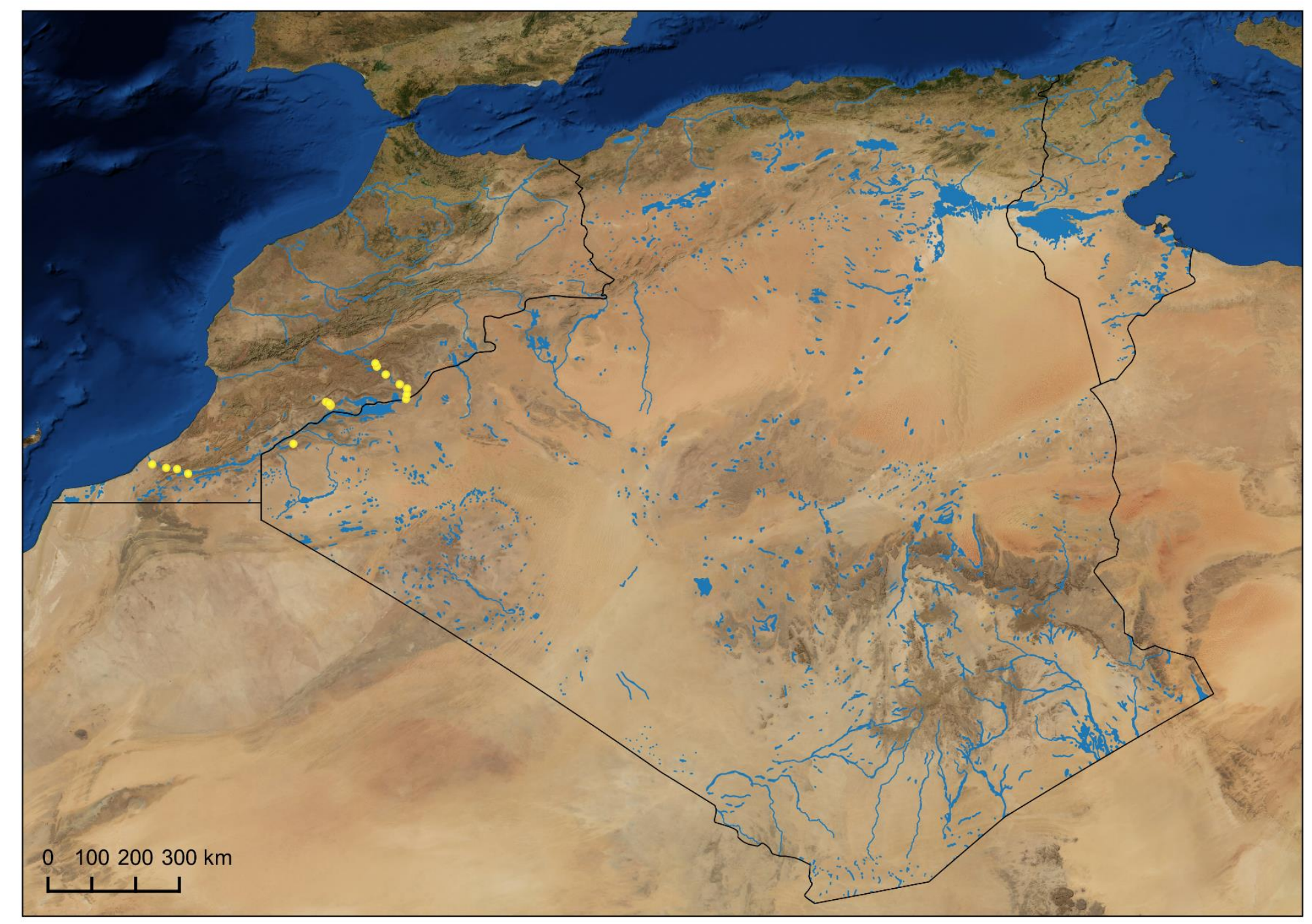

Figure 82: Oreochromis aureus. 


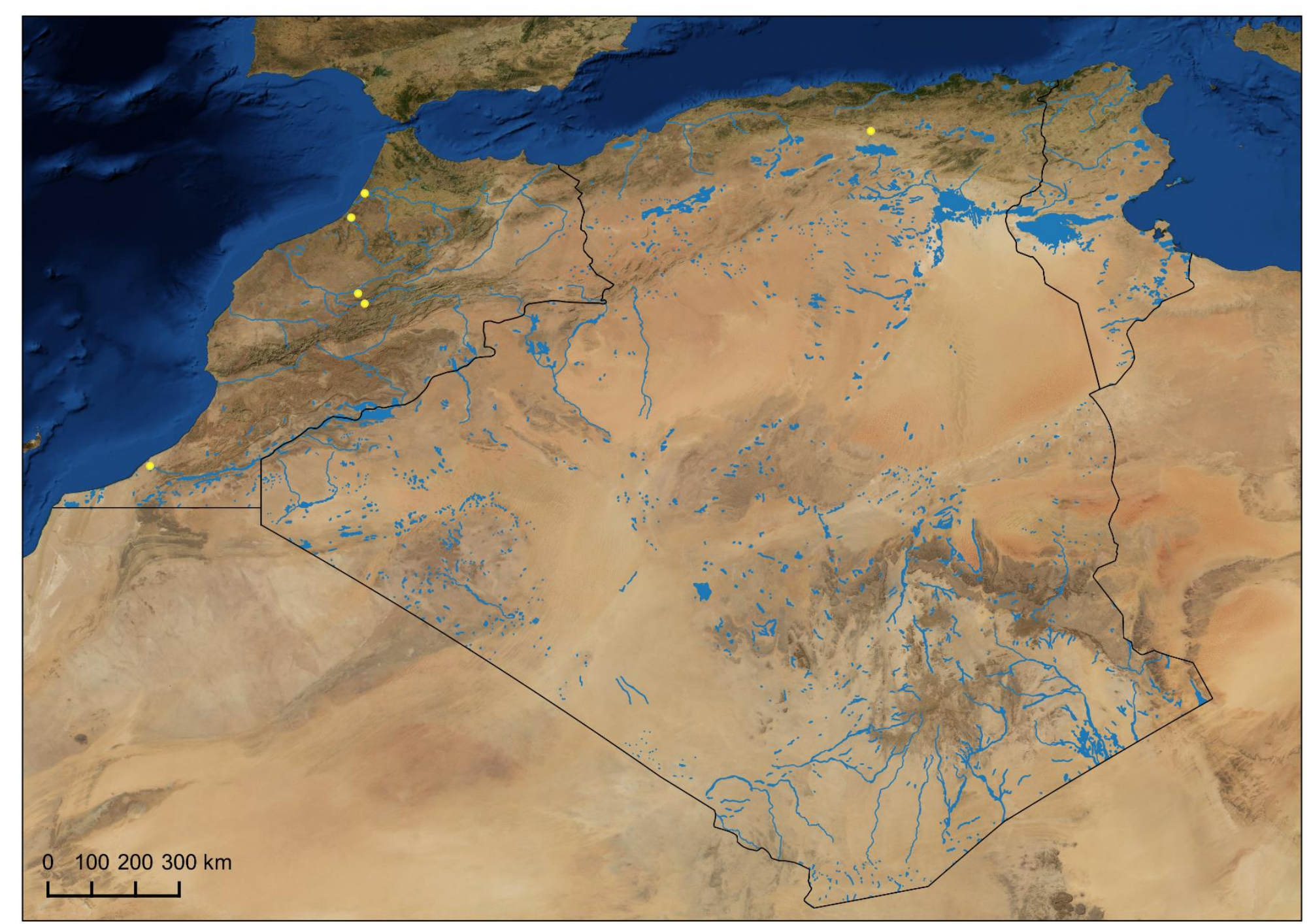

Figure 83: Oreochromis niloticus. 


\section{Family: Percidae}

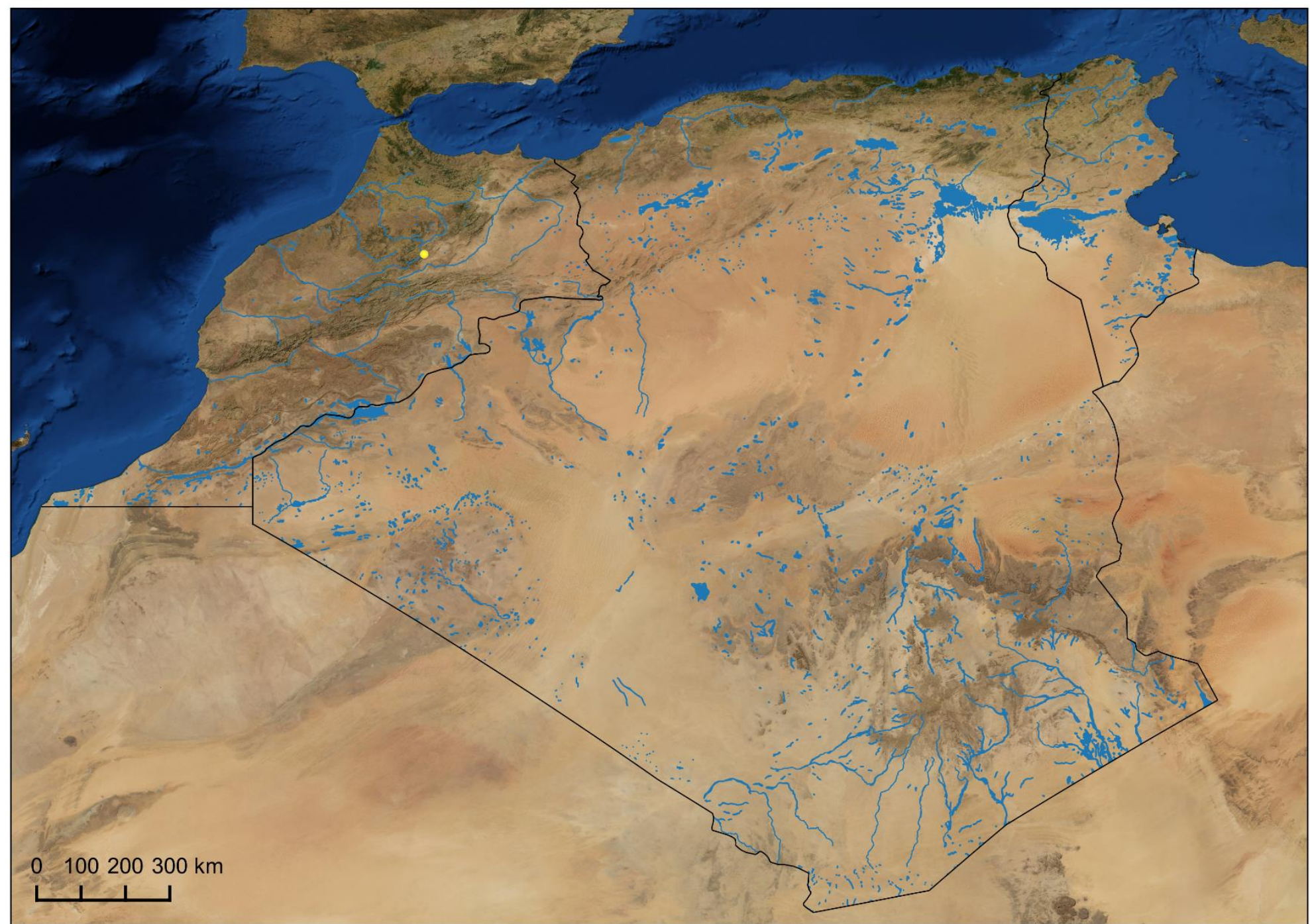

Figure 84: Perca fluviatilis. Recently recorded in the region for the first time and may occur elsewhere. 


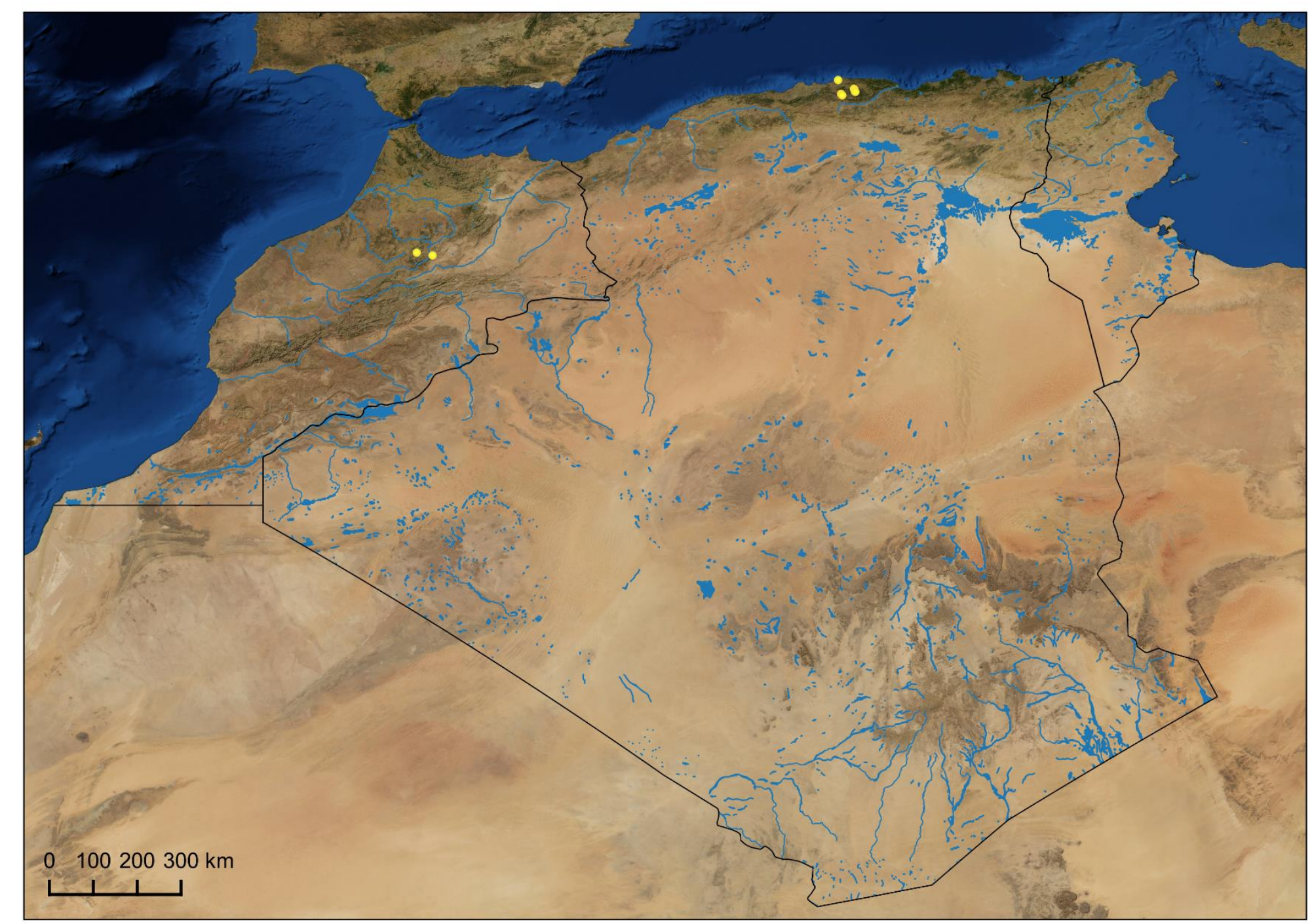

Figure 85: Sander lucioperca. Likely to be more widespread in the region. 


\section{Family: Blenniidae}

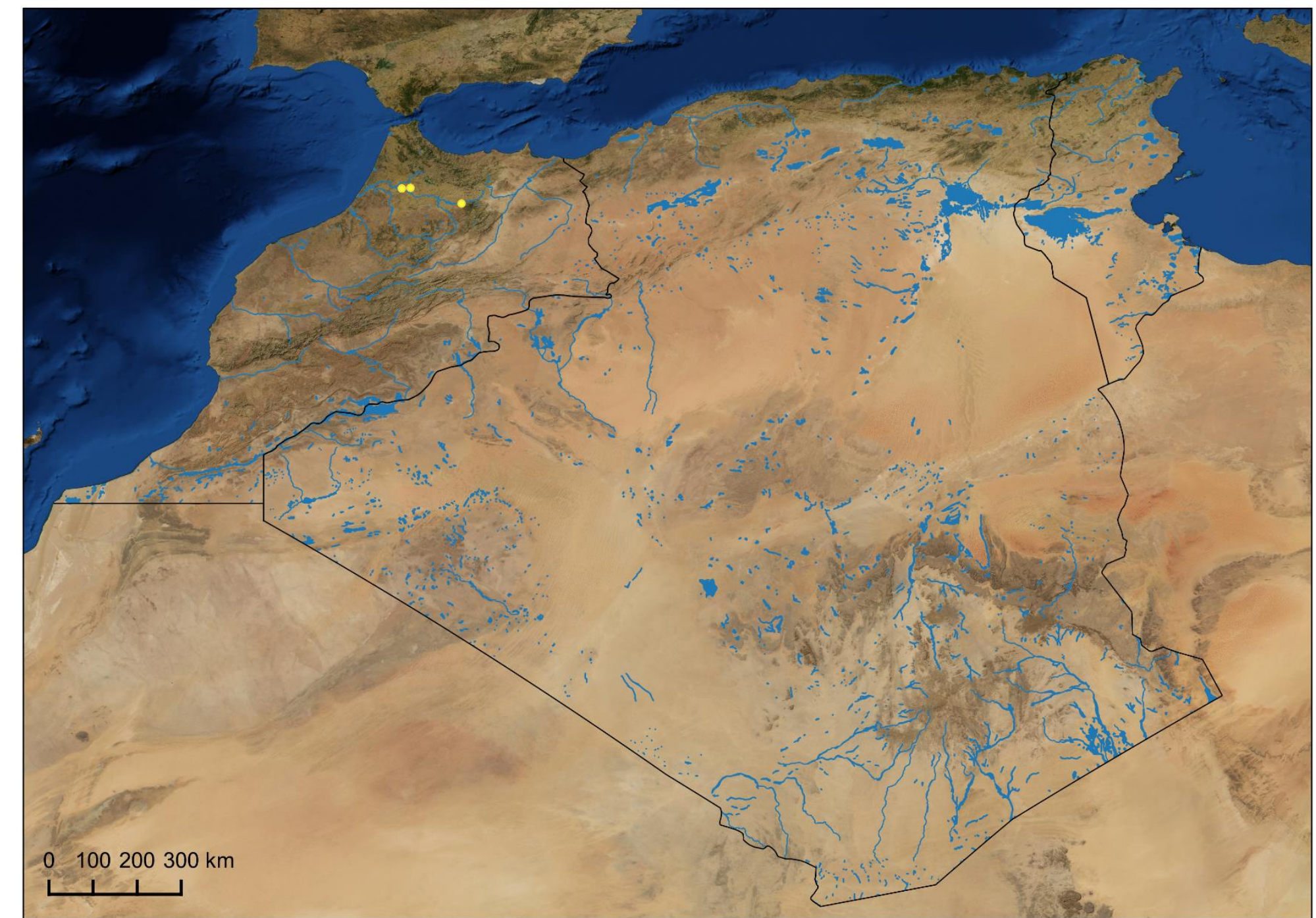

Figure 86: Salaria atlantica. 


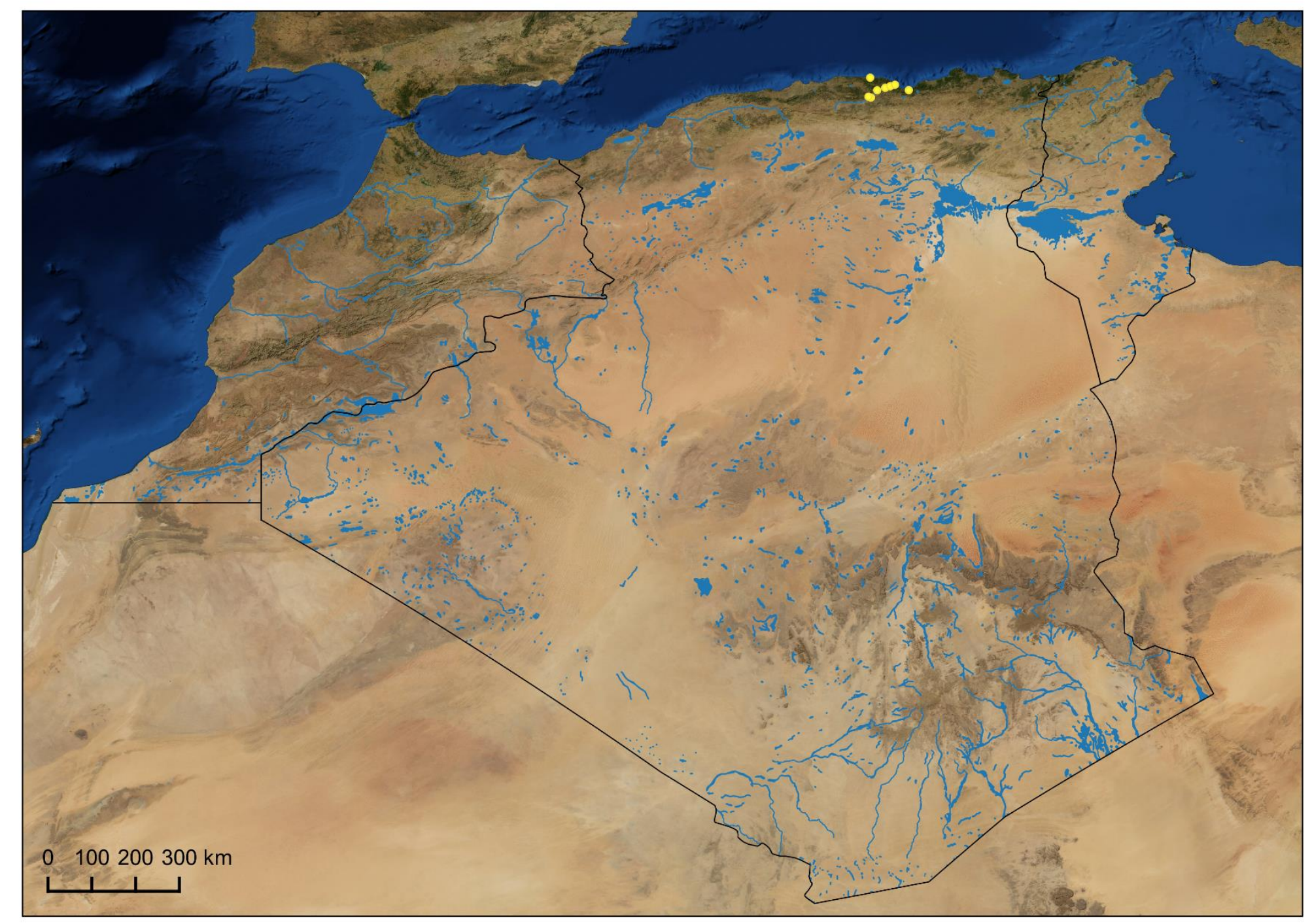

Figure 87: Salaria fluviatilis. 